\title{
Action des courants, de la houle el du vent sur les sédiments
}

\section{Claude Migniol}

Ingénieur en Chef du département de Sédimentologie

du LABORATOIRE CENTRAL D'HYDRAULIQUE DE FRANCE

\section{Introduction}

Sous l'action des courants, des vents ou des houles, les particules solides, qui forment les sédiments que l'on rencontre dans les fleuves et le long des littoraux, peuvent être arrachées des fonds, entraînées en suspension ou en charriage sur des distances plus ou moins grandes et déposées dans les zones calmes.

Ces remaniements sédimentaires provoquent, dans certains cas, des érosions ou des dépôts préjudiciables à l'équilibre naturel d'un site, ou à son aménagement, et il est nécessaire avant d'aborder la réalisation d'un ouvrage de tenir compte des interactions possibles entre les fluides en mouvement et les sols sur lesquels ils coulent.

Ces interactions sont extrêmement complexes en nature et les mouvements sédimentaires que l'on peut constater dépendent de multiples paramètres. Gradients de vitesses dans le fluide, tourbillons, géométrie des fonds, tracés des rives, nature des matériaux, épaisseur sur le substratum, porosité et cohésion des dépôts, caractéristiques du fluide. . . vont intervenir dans les conditions d'érosion et de transport des matériaux. Tous ces paramètres, en plus, ne seront pas constants dans le temps mais subiront des fluctuations et l'équilibre sédimentaire final ne sera qu'une résultante d'actions hydrodynamiques où fluides et solides réagissent l'un sur l'autre.

Les lois exactes qui régissent ces phénomènes restent encore imparfaitement connues et il est difficile, voire impossible, par une simple approche théorique des mouvements sédimentaires de prévoir avec précision l'évolution d'un site soumis à des actions hydrodynamiques multiples.

Il est par contre possible, en utilisant un certain nom- bre de lois théoriques et empiriques de mieux comprendre les phénomènes physiques qui vont contribuer aux mouvements sédimentaires et de quantifier l'importance de ces mouvements dans des cas relativement simples.

Dans cet exposé nous limiterons la théorie au minimum et essaierons, avant tout, de donner des éléments pratiques qui permettent de définir les ordres de grandeur des mouvements sédimentaires.

Aprés avoir rappelé les propriétés physiques des particules solides dans une eau calme, leur comportement sous l'action des courants sera abordé avant d'examiner plus en détail l'action de la houle sur différents types de matériaux et l'importance des transports éoliens.

\section{Propriétés physiques des particules solides en eau calme}

\section{Particules élémentaires - Vitesses de chute}

Une particule élémentaire est caractérisée par son diamètre, sa densité, sa forme et son état de surface. On peut également définir son volume et son maitre couple, c'est-à-dire sa surface portante lorsqu'elle tombe dans un fluide.

Ces caractéristiques se trouveront réunies dans la vitesse de chute des particules, élément fondamental pour mieux comprendre leur comportement sous une action hydrodynamique.

Dans une eau calme, une particule élémentaire est soumise à trois forces : force de gravité (proportionnelle à la masse de la particule), poussée d'Archimède. 
résistance opposée par le fluide au mouvement de la particule.

Suivant que le mouvement relatif du fluide par rapport à la particule est lent, moyen ou rapide, l'écoulement sera laminaire (régime de Stokes), semi-turbulent (régime d'Allen) ou turbulent (régime de NewtonRittinger). Ces différents régimes correspondent à des nombres de Reynolds ( $R \epsilon=\mathrm{VH}^{\prime} \nu$ ) déterminés (fig. 1 ). Rappelons que le nombre de Reynolds est le rapport des forces d'inertie aux forces de frottements visqueux : dans le cas d'une particule tombant dans de l'eau, par exemple, on aura $R e=W d / v, W$ étant la vitesse de chute, $d$ le diamètre de la particule et $\nu$ la viscosité cinématique de l'eau (égale à $20^{\circ} \mathrm{C}$ à $10^{-6} \mathrm{~m}^{2} / \mathrm{s}$ et à $3,5^{\circ} \mathrm{C}$ à $1,610^{-6} \mathrm{~m}^{2} / \mathrm{s}$ ).
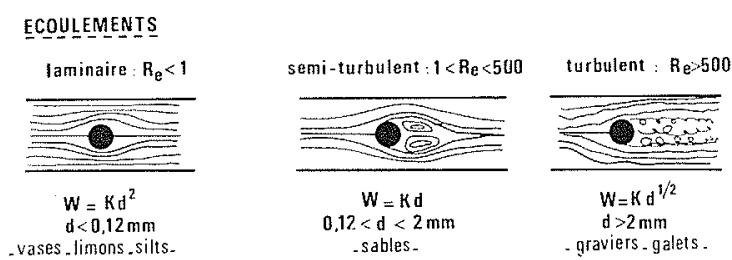

Figure 1 - Les différents types d'écoulement

\section{Ecoulement laminaire (loi de Stockes)}

$$
W=K d^{2}=\frac{1}{18}\left(\frac{\rho_{s}-\rho_{o}}{\rho_{o}}\right) d^{2} g / v
$$

$\rho_{s}$ et $\rho_{o}$ étant la masse volumique du solide et du fluide (en $\mathrm{Kg} / \mathrm{m}^{3}$ ou en $\mathrm{g} / \mathrm{cm}^{3}$ )

Dans la pratique, on pourra obtenir une prévision ac. ceptable en admettant que (dans une eau à $20^{\circ} \mathrm{C}$ ) on a :

\begin{tabular}{|c|c|}
\hline $\begin{array}{c}\text { Sédiments nature/s } \\
\text { (densité } 2,55) \\
\text { (silts, argile, vase) }\end{array}$ & $\begin{array}{c}\text { Matériaux artificie/s } \\
\text { (densité } 1,40) \\
\text { (bakélite inf. à } 0,15 \mathrm{~mm})\end{array}$ \\
\hline $\begin{array}{c}\text { Diamètre en } \mathrm{cm}= \\
=\frac{(W(\mathrm{~cm} / \mathrm{s}))^{1 / 2}}{100}\end{array}$ & $\begin{array}{c}\text { Rayon en } \mathrm{cm}= \\
=\frac{(W(\mathrm{en} \mathrm{cm} / \mathrm{s}))^{1 / 2}}{100}\end{array}$ \\
\hline
\end{tabular}

Par exemple si pour une particule de silt ou de limon la vitesse de chute est de $0,1 \mathrm{~cm} / \mathrm{s}$, le diamètre équivalent de la particule sera de $0,00315 \mathrm{~cm}=31,5$ microns. Si nous avions eu la même vitesse de chute pour une particule de bakélite, nous aurions eu un diamètre équivalent de 63 microns, c'est-à-dire 2 fois plus gros.

Notons l'influence de la viscosité de l'eau sur la vitesse de chute des particules; la vitesse de chute pourra être réduite de moitié lorsque la température de l'eau passe de $30^{\circ} \mathrm{C}\left(\nu=0,8.10^{-6} \mathrm{~m}^{2} / \mathrm{s}\right)$ à $4^{\circ} \mathrm{C}\left(\nu=1,6.10^{-6}\right.$ $\left.\mathrm{m}^{2} / \mathrm{s}\right)$. En nature la décantation risque de ne pas être la même en été et en hiver pour de mêmes éléments fins.

\section{Ecoulement semi-turbulent (sables)}

$$
W=K d
$$

De nombreuses mesures ont été faites au LCHF sur des matériaux naturels et artificiels ayant des gammes de densités comprises entre 2,6 et $1,045(2,6-2-1,6-$ $1,4-1,12-1,045)$. Des abaques permettent d'avoir directement les vitesses de chute (figure 2).

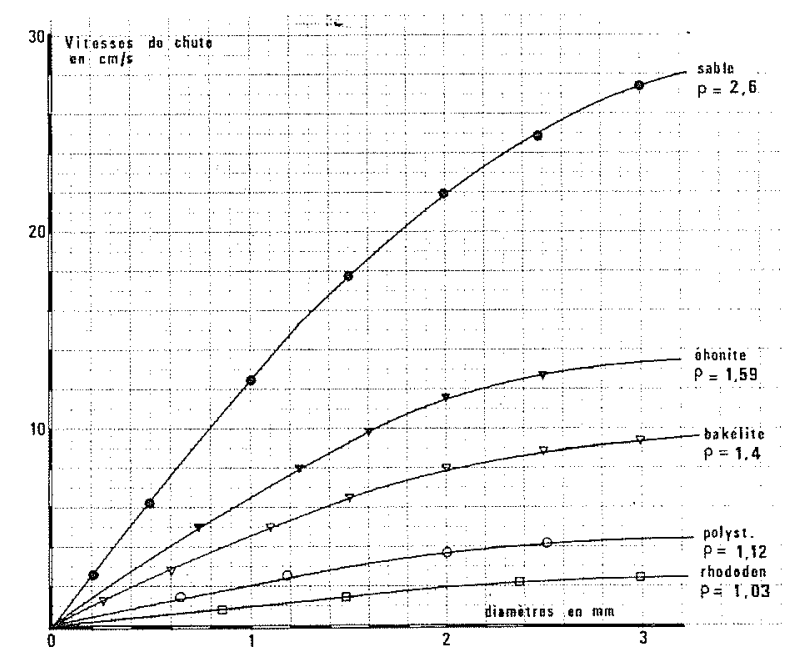

Figure $2-$ Vitesses de chute de différents types de matériaux

Dans la pratique on pourra avoir une idée des vitesses de chute de quelques matériaux (pour les diamètres compris entre 0,2 et $1 \mathrm{~mm}$ ) en prenant l'expression simplifiée :

\begin{tabular}{|c|c|c|}
\hline $\begin{array}{c}\text { Sédiments nature } / \mathrm{s} \\
\left(\rho=2,60 \mathrm{~kg} / \mathrm{m}^{3}\right)\end{array}$ & $\begin{array}{c}\text { Bakélite } \\
\left(\rho=1,40 \mathrm{~kg} / \mathrm{m}^{3}\right)\end{array}$ & $\begin{array}{c}\text { Polystyrène } \\
\left(\rho=1,12 \mathrm{~kg} / \mathrm{m}^{3}\right)\end{array}$ \\
\hline $125 \mathrm{~d}$ & $45 \mathrm{~d}$ & $20 \mathrm{~d}$ \\
\hline
\end{tabular}

Autrement dit une particule de bakélite (comprise entre 0,2 et $1 \mathrm{~mm}$ ) aura, à diamètre égal, une vitesse de chute 3 fois plus faible qu'une particule de sable. Une particule de polystyrène aurait une vitesse de chute 6 à 7 fois plus faible.

\section{Ecoulement turbulent (galets)}

$$
W=K d^{1 / 2}
$$

avec : $\quad K=70\left(\frac{\rho_{s}-\rho_{0}}{\rho_{0}}\right)^{1 / 2}$ en c.g.s.

Un galet de $5 \mathrm{~cm}$ aurait une vitesse de chute de près de $2 \mathrm{~m} / \mathrm{s}$.

Pour de tels matériaux, il faudra également avoir à l'esprit que leurs dimensions les mettent en dehors de la couche limite sur les fonds.

Précisons également que pour toutes les particules la forme est extrêmement importante. En particulier pour des coquilles plates la vitesse de chute tend vers une valeur asymptotique lorsque le diamètre augmente. 


\section{Ensemble de particules - Vitesses de chute}

Si des particules tombent simultanément dans un fluide elles pourront se gêner - La vitesse de chute sera entravée. Par ailleurs, si elles sont très fines elles pourront s'agglomérer entre elles - il y aura floculation.

\section{Vitesses de chute entravée}

La vitesse de chute de particules supérieures à $100 \mathrm{mi}$ crons $(0,1 \mathrm{~mm})$ diminuera lorsque la concentration de la suspension augmentera.

Par exemple pour une concentration de sable fin de $100 \mathrm{~g} / 1$, la vitesse de chute des particules sera égale à 0,75 fois celle mesurée sur une particule élémentaire.

Pour de la bakélite la vitesse de chute d'une suspension à $100 \mathrm{~g} / 1$ ne serait que de 0,5 fois celle mesurée sur la particule élémentaire.

Pour des particules fines en milieu défloculé, la vitesse de chute serait encore plus réduite, elle ne serait par exemple que de 0,15 fois celle mesurée sur une particule élémentaire pour une concentration de $100 \mathrm{~g} / 1$ de poudre de bakélite de 40 microns (au lieu de 0,5 fois pour des grosses particules).

Là encore des études systématiques faites en laboratoire permettent d'avoir une idée des phénomènes (figure 3).

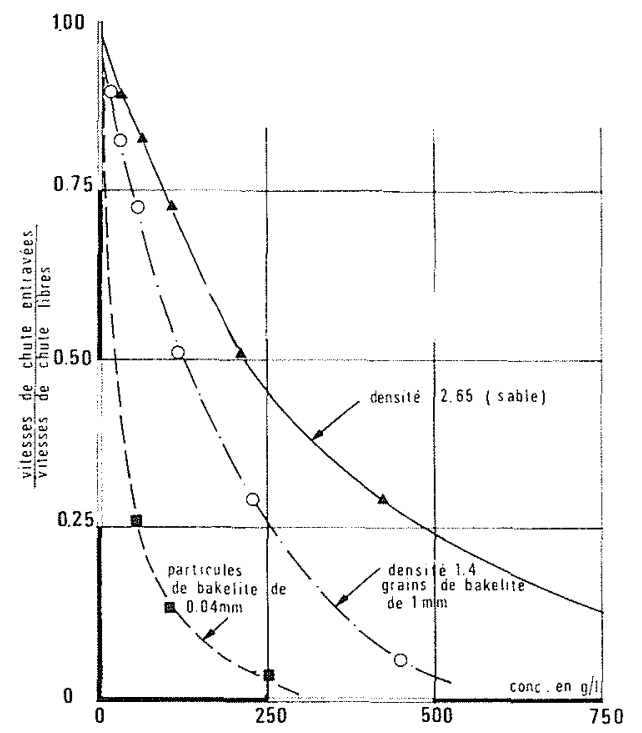

Figure 3 -. Influence de la concentration des particules solides sur la vitesse de chute entravée (đ'après Gandin)

\section{Floculation des fines particules - Influence du milieu a- queux}

Si sur de grosses particules on constate que la vitesse de chute est diminuée lorsque la concentration de la suspension augmente, on note sur des éléments pélitiques très fins (poudres, limons, vases et argiles) que le phénomène est plus complexe par suite de la floculation.
La floculation est la propriété, pour les fines particules, de se prendre en agrégats de dimension très supérieure à celles des éléments qui la constituent (agrégats $=0,1$ à $2 \mathrm{~mm}$, particules quelques microns).

Alors qu'une particule élémentaire de vase de $1 \mathrm{mi}$ cron a une vitesse de chute de $0,001 \mathrm{~mm} / \mathrm{s}$, c'est-à-dire qu'elle mettra 120 jours pour parcourir en eau calme une hauteur de $10 \mathrm{~m}$, elle aura à l'état floculé une vitesse moyenne de chute comprise entre 0,1 et $0,8 \mathrm{~mm} / \mathrm{s}$ et pourra donc se décanter sur une hauteur de $10 \mathrm{~m}$ en $5 \mathrm{~h}$. environ.

La floculation et la vitesse de chute varieront en fonction :

_ de la concentration en particules solides (figure 4).

La vitesse de chute augmentera progressivement entre

0 et 15 à $20 \mathrm{~g} / 1$ de concentration puis rediminuera ensuite (vitesse entravée) ;

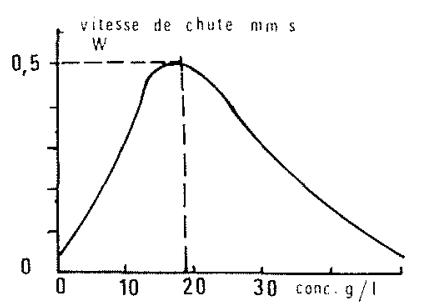

Figure 4 - Influence de la concentration sur la vitesse do chute

de la salinité des eaux (fig. 5). La vitesse de chute augmentera lorsque la salinité passera de 0 à $5 \% 0$, elle sera constante au-delà ou en légère diminution (macrofloculation).

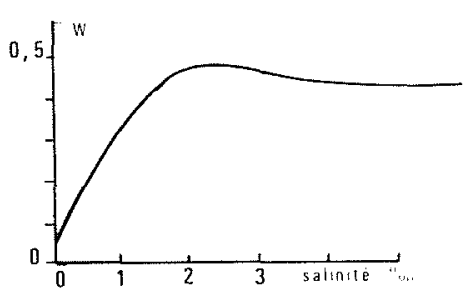

Figure 5 - Influence de la salinité sur la vitesse de chute

En pratique on pourra admettre qu'en nature les vases floculées ont des vitesses de chute de l'ordre de $0,5 \mathrm{~mm} / \mathrm{s}$ en eau calme. Cette vitesse de chute pourra être réduite, si, par suite de la turbulence, les flocons se déchirent; au contraire elle pourra être augmentée si sous l'effet d'un léger brassage des macroflocons se produisent (cas des bassins de décantation).

On constate d'ailleurs en nature, notamment dans des estuaires, que la vitesse de chute des macroflocons qui se forment au voisinage des étales, peut atteindre 1 à $2 \mathrm{~mm} / \mathrm{s}$ (mesures faites par traceurs radioactifs).

D’une façon générale l'intensité de la floculation dépendra :

- des caractéristiques des particules : dimension, nature chimique et minéralogique, charge électrique (potentiel)...;

-.. des caractéristiques du milieu : concentration électrolytique, valence des ions, température. 

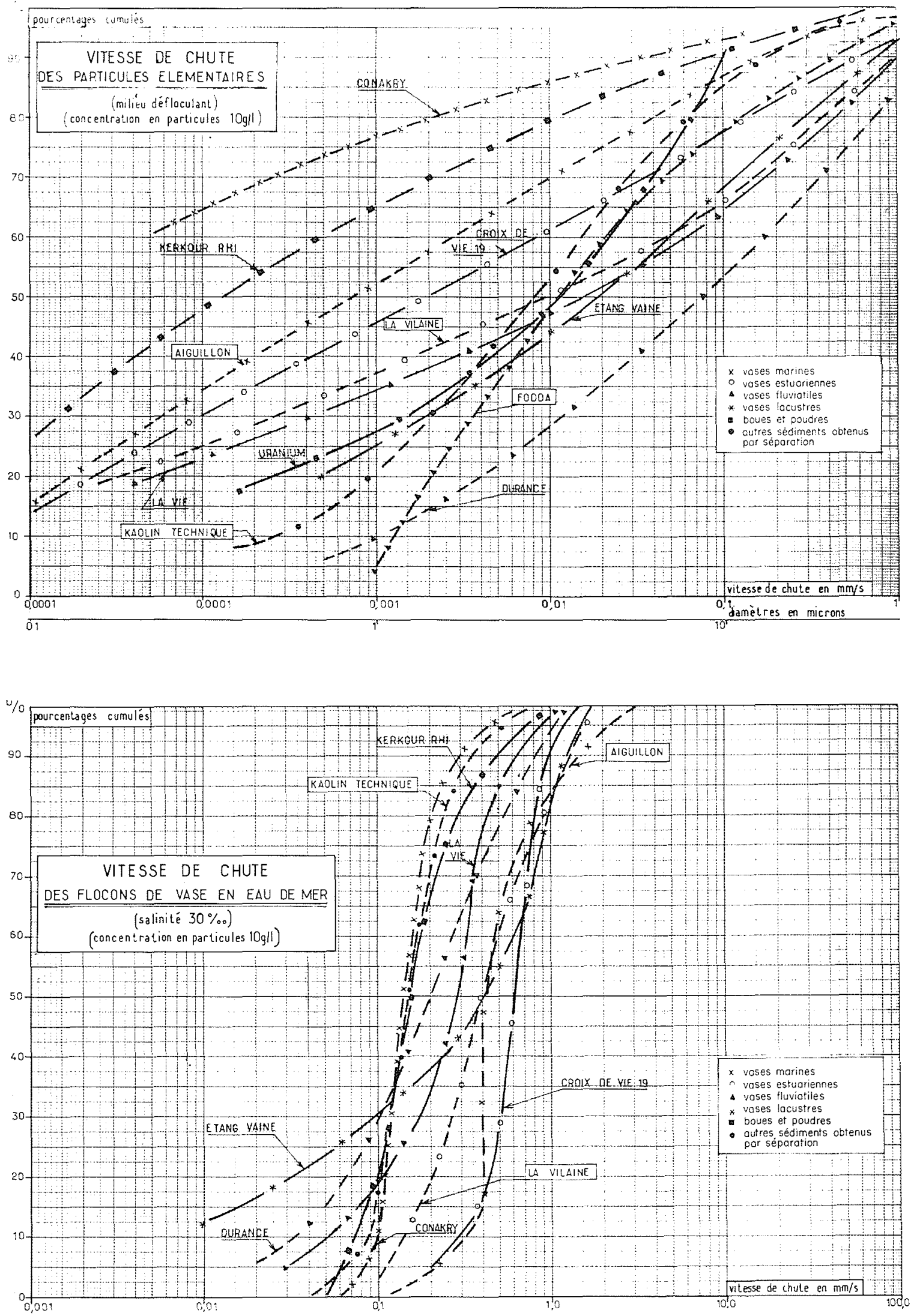

Figure $6 \mathrm{a}, \mathrm{b}-$ Vitesse de chute des particules élémentaires et des flocons 
D'après les mesures faites au L.C.H.F. on peut dire que le facteur de floculation $F$, que l'on peut définir comme le rapport entre la vitesse de chute libre des flocons $W f 50 \%$ et la vitesse moyenne de chute des particules élémentaires $W d 50 \%$, est d'autant plus grand que les particules élémentaires sont plus petites :

$$
F=W f 50 \% / W d 50 \% \cong 250 D^{-1,8}=W d^{-0,9}
$$

Ce fait se conçoit aisément si l'on considère que plus les particules sont petites, plus la surface totale de celles-

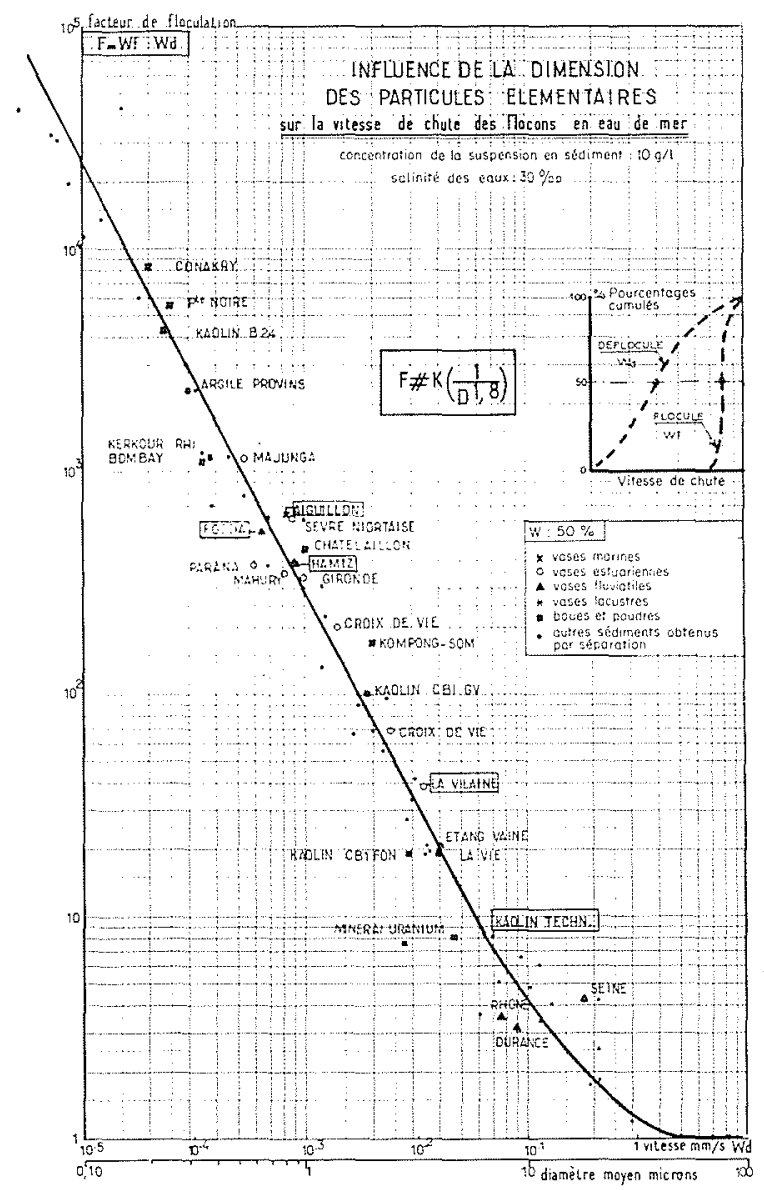

Figure 7 - Variation du facteur de floculation en fonction de la dimension des particules

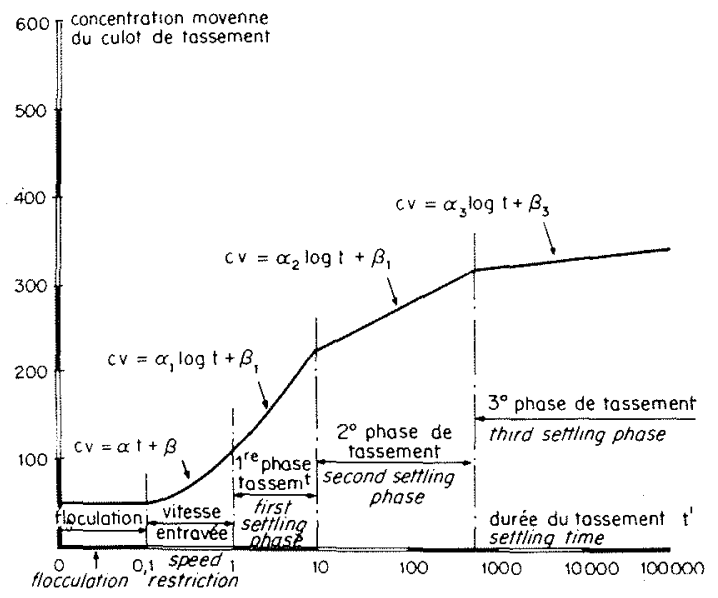

Figure 8 - Variation de la concentration en fonction du temps ci, à poids égal, est grande et plus l'action de floculation est importante.

Le tableau ci-après et les figures 5 - 6 et 7 ci-joints illustrent ces résultats et donnent quelques valeurs du facteur de floculation $F$ en fonction du diamètre de la particule élémentaire $(D)$.

\begin{tabular}{|l|c|r|r|r|}
\hline D en microns & 0,1 & 1 & 10 & 40 \\
\hline$F$ & 20.000 & 500 & 5 & 1 \\
\hline
\end{tabular}

\section{Tassement des matériaux}

\section{Sédiments grossiers (sables - graviers - cailloux)}

Les sédiments grossiers du type sables, galets et graviers une fois déposés sur les fonds ne se tassent pratiquement pas. Si ils subissent une "mise en place" et une imbrication entre eux, on peut admettre qu"ils sont stables dans le temps.

\section{Sédiments fins (limons - vases - argiles)}

Les éléments fins par contre sont essentiellement variables dans le temps et se tassent en perdant leur eau interstitielle. Ils se consolident et passent d'un état fluide à un état plastique, puis solide (Voir La Houille Blanche, $n^{\circ} 7 / 1968$ ).

Sans entrer dans le détail de cette consolidation, on peut dire que :

- la concentration moyenne $T_{s}$ du dépôt varie comme le logarithme du temps $t$ (figure 8 ):

$$
T_{s}(g / l)=\alpha \log t+\beta
$$

- il existe un gradient important de concentration dans les dépôts entre la surface et le fond $H$ tel que (figure 9):

$$
T_{s(H)}=T_{s(S)}+n \log H
$$

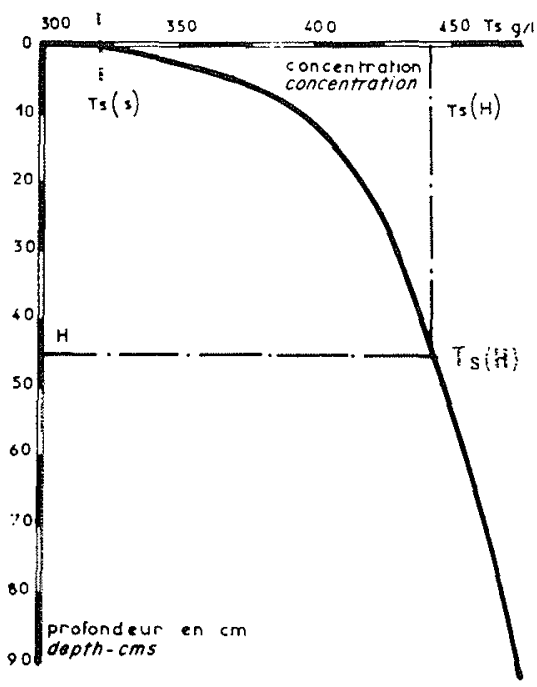

Figure 9 - Variation de la concentration en fonction de la profondeur 
- le tassement est très différent suivant les matériaux et la nature des eaux, certains dépôts restent fluides pendant plusieurs mois (exemple : boues de lavage des phosphates) alors que d'autres se consolident en quelques jours (limons de Fos) ;

- il est possible de modifier le tassement en agissant sur le milieu aqueux ou sur la charge électrique des particules. De même l'épaisseur du dépôt a une action importante sur le tassement.

\section{Cohésion et rhéologie des dépôts}

\section{Sédiments grossiers}

Les sédiments grossiers n'ont pas de cohésion propre mais leur équilibre dépend de leur angle de frottement interne $\Phi$ qui peut être déduit des contraintes normales on et tangentielles $\sigma t$ :

$$
\sigma t=\operatorname{tg} \Phi \text { on }
$$

Pour les matériaux uniformes (D60/D10<2) jl y a une relation de première approximation entre $\operatorname{tg} \phi$ et l'indice des vides e (sédiments hors d'eau) :

$$
\operatorname{tg} \Phi=0,55 / e
$$

On admet en général les valeurs suivantes pour dif. férents types de sédiments grossiers hors d'eau.

\begin{tabular}{|l|c|c|c|c|c|}
\hline Nature & $\begin{array}{c}\text { Sable } \\
\text { plage }\end{array}$ & $\begin{array}{c}\text { Sable } \\
\text { roulé }\end{array}$ & $\begin{array}{c}\text { Sable } \\
\text { serré }\end{array}$ & Gravier & $\begin{array}{c}\text { Débris } \\
\text { rocheux }\end{array}$ \\
\hline $\begin{array}{l}\text { Densité } \\
\text { apparente } \\
\left(\mathrm{g} / \mathrm{cm}^{3}\right)\end{array}$ & 1,6 & 1,7 & 1,8 & 1,8 & 1,8 \\
\hline $\begin{array}{l}\Phi \text { en } \\
\text { degrés }\end{array}$ & $\begin{array}{c}25 \text { à } \\
30\end{array}$ & $\begin{array}{c}28 \grave{a} \\
32\end{array}$ & $\begin{array}{c}34 \grave{a} \\
38\end{array}$ & $\begin{array}{c}35 \text { à } \\
40\end{array}$ & $\begin{array}{c}40 \text { à } \\
50\end{array}$ \\
\hline
\end{tabular}

\section{Sédiments fins}

Les éléments fins sont beaucoup plus complexes et présentent une cohésion d'autant plus grande que leur concentration en particules est plus forte et le milieu aqueux plus floculant.

Pour quantifier l'état du dépôt on procède en laboratoire à la mesure de sa "rigidité" qui permettra de prévoir son comportement sous des actions hydrodynamiques.

Cette mesure s'effectue en définissant le couple nécessaire pour faire tourner un objet dans la suspension ou le dépôt.

On constate que d'une façon schématique les dépôts d'éléments pélitiques ont des rigidités $\tau y$ qui varient très rapidement avec la concentration (figure 10) :

$$
\tau y=n T_{s}^{4} \text { à } 5
$$

Suivant la nature du dépôt la valeur de la rigidité pourra être très différente. De même, le milieu aqueux modifiera, pour une même concentration en particules, la rigidité des dépôts.

Il semble que viscosité et rigidité initiale soient d'autant plus faibles que la granulométrie des particules élémentaires est grande.

A titre d'exemple, une poudre calcaire aura pour une mème concentration en particules solides de la suspension $(400 \mathrm{~g} / 1)$ une rigidité initiale 1000 fois plus faible qu'une vase de La Vilaine.

En mettant $8 \mathrm{mg}$ de pyrophosphate par gramme de sédiment on obtiendra après 3 mois une concentration moyenne des dépôts de $240 \mathrm{~g} / 1$ alors que sans pyrophosphate on obtenait $430 \mathrm{~g} / 1$ (concentration initiale de Kaolin à $200 \mathrm{~g} / 1$ ).

Parallèlement, et pour une même concentration des matériaux, la rigidité mesurée sera 5 à 10 fois plus faible.

\section{Pente d'équilibre des dépôts}

La pente des dépôts est également une caractéristique importante des matériaux.

\section{Sédiments grossiers}

Pour des éléments grossiers, n'ayant pas de rigidité initiale propre ou de cohésion, cette pente ne sera autre que l'angle de frottement du matériau. A titre d'exemple si l'on prend un sable et si on le dépose sous l'eau en un point déterminé il se mettra sous la forme d'un cône dont l'angle du talus pourra être précisé.

Pour du sable et des petits graviers de silice (densité 2,60 ), la pente du talus sous l'eau varie entre $31^{\circ}$ $(\operatorname{tg} \alpha=0,6)$ pour des éléments fins de $0,14 \mathrm{~mm}$ de diamètre et $40^{\circ}(\operatorname{tg} \alpha=0,84)$ pour les éléments de $5,5 \mathrm{~mm}$.

Pour de la bakélite (densité 1,40 ), la pente varie entre $34^{\circ}$ pour les éléments de $0,14 \mathrm{~mm}$ et $46^{\circ}$ pour les élements de $5 \mathrm{~mm}$; elle est en moyenne un peu plus forte que pour les sables.

D'une façon plus générale on admet que sous l'eau :

- la pente d'équilibre du dépôt croît comme la racine carrée du diamètre des éléments moyens;

- lorsque le matériau est homogène et isotrope, l'angle de stabilité $\theta$ est inférieur à l'angle de frottement interne $\Phi$, on a :

$$
\operatorname{tg} \theta=\frac{\rho s \operatorname{tg} \Phi}{\rho s+n \rho}
$$

$n$ étant la porosité du dépôt (volume des vides : vo. lume total, variant de 26 à $48 \%$ )

a la masse spécifique de l'eau et $\rho s$ la masse spécifique du sédiment

A partir d'abaques, donnés ci-dessous (figures 11 et 12), on peut déterminer $\theta$ en connaissant la valeur du paramètre :

$$
f=n \frac{\rho}{\rho s}
$$


100 prigidite initiale en Newton $/ m^{2}$

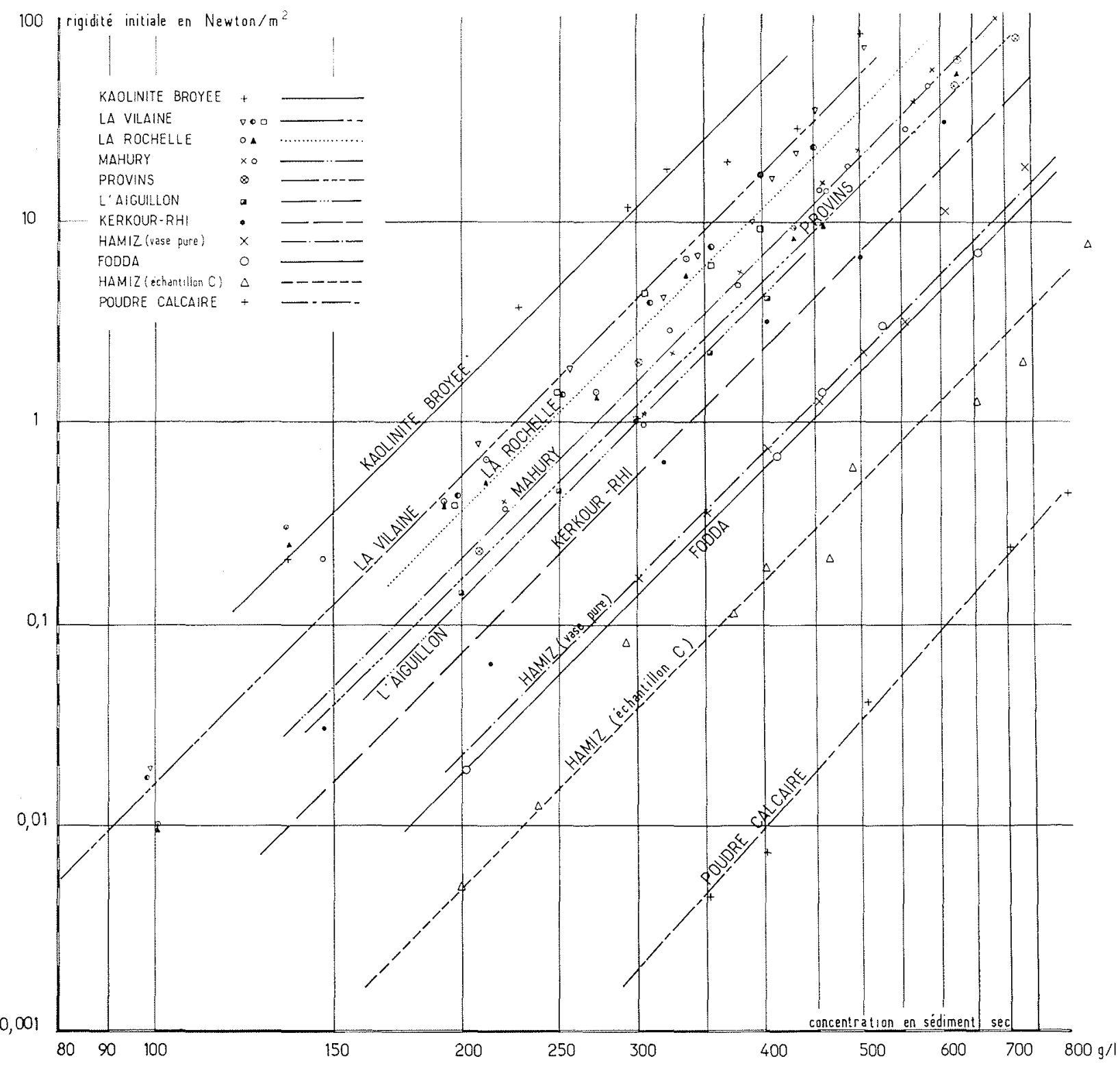

Figure 10 - Variation de la rigidité initiale en fonction de la concentration

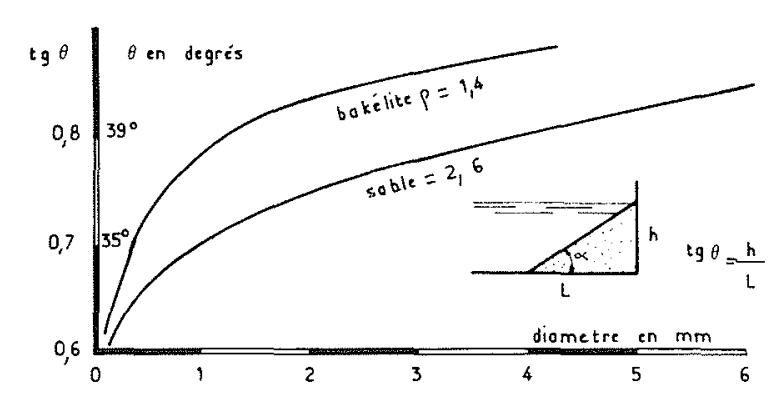

Figure 11 - Exemple de pentes d'équilibre d'un talus

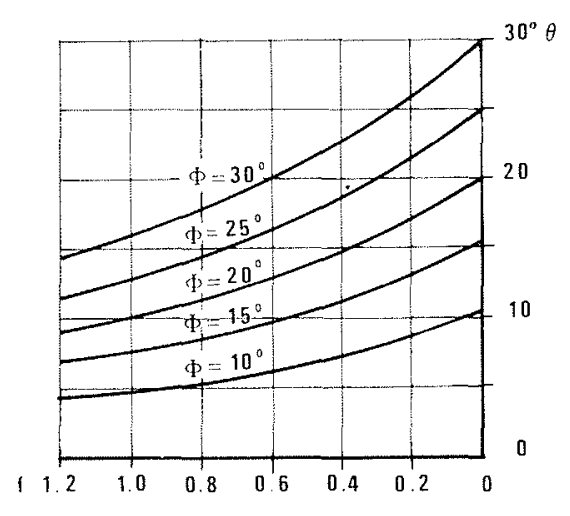

Figure 12 - Variation de l'angle de talus sous l'eau $\theta$ en fonction de l'angle de frottement interne $\Phi$ 
La pente est plus forte lorsque la densité du matériau diminue.

\section{Sédiments fins}

Pour les sédiments fins, ayant une rigidité initiale, la pente dépendra, d'une part, de la concentration en sédiments $\left(T_{s}\right)$, d'autre part, de la nature des sédiments et du milieu aqueux (Fig. 13-14). On constate que la pente d'un talus se rattache à la rigidité initiale $\tau y$ telle que :

- talus émergé : $\operatorname{tg} \theta=0,007 \tau y\left(\right.$ en $\left.\mathrm{N} / \mathrm{m}^{2}\right)$

- talus immergé : $\operatorname{tg} \alpha=0,025 \tau y\left(\right.$ en $\left.\mathrm{N} / \mathrm{m}^{2}\right)$

Précisons que si la vase contient des particules de sable, la rigidité et la pente d'équilibre ne sont que faiblement modifiées si la teneur en eau de la vase qui entoure les grains reste constante. En fait pour un pourcentage de sable inférieur à $30 \%$, seules les propriétés de la vase ont une influence.
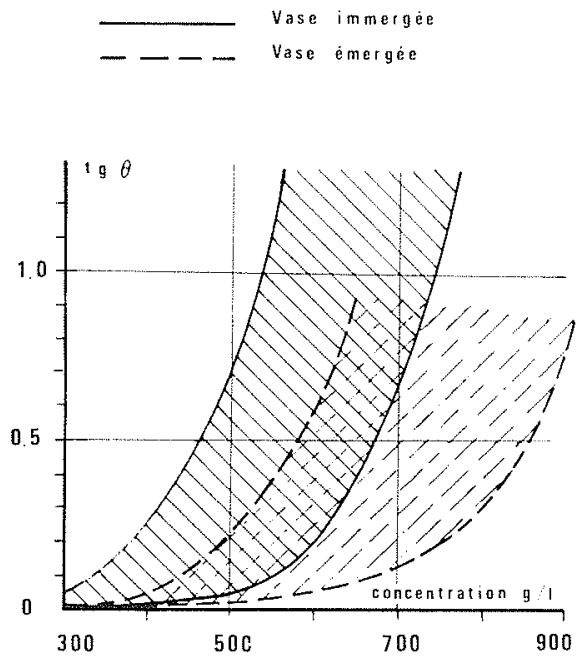

Figure 13 - Pente d'équilibre des vases immergées et émergées

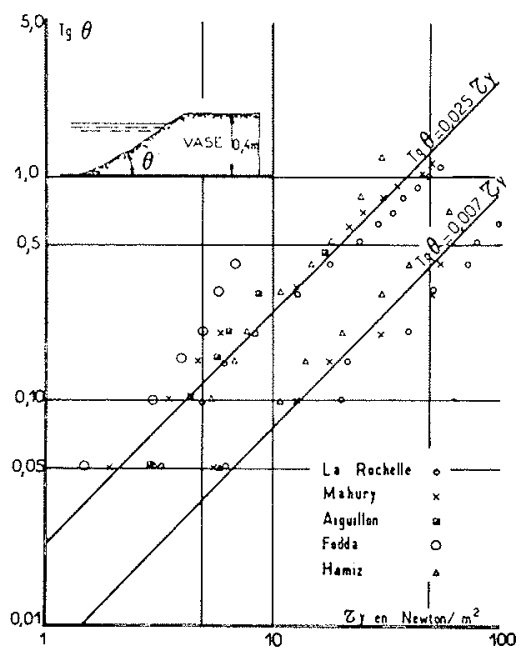

Figure 14 - Influence de la rigidité initiale sur la pente d'équilibre d'un talus de vase

\section{Comportement des sédiments dans un courant}

\section{Généralités}

Une particule sédimentaire déposée sur les fonds ne peut être mise en mouvement que si les forces hydrodynamiques qui agissent sur elle sont supérieures aux forces stabilisatrices de la pesanteur propres à l'élément intéressé et à son imbrication dans l'ensemble des éléments. Pour les éléments fins, il faut en plus tenir compte des effets de cohésion entre particules.

Les particules étant en mouvement, le débit de charriage sera d'autant plus important que les actions hydrodynamiques seront intenses.

Il ne saurait être question de traiter ici toutes les formules de transport établies, elles sont en effet fort nombreuses. Nous rappellerons simplement les quatre critères fondamentaux auxquels devrait répondre une bonne formule de transport :

- la formule doit décrire aussi bien que possible toutes les phases du transport. Les résultats doivent être exacts aussi bien pour les petits que pour les moyens et grands transports ;

- la formule doit être utilisable pour un domaine aussi grand que possible, c'est-à-dire aussi bien pour les mesures en laboratoire que pour les cours d'eau naturels, pour les matériaux légers que pour les matériaux lourds, pour des matériaux uniformes que pour des matériaux à granulométrie étendue. Cette condition est surtout importante pour la technique des modèles réduits ;

- la formule doit être de construction simple et doit pouvoir s'employer de manière aisée sans trop de diagrammes et sans calculs compliqués parce que l'exactitude que l'on peut ainsi atteindre n'est qu'apparente par rapport à l'incertitude des données;

- la formule doit être homogène, c'est-à-dire que les dimensions des différents éléments doivent correspondre les unes aux autres et les constantes qui ont une dimension doivent être éliminées.

\section{Paramètres hydrauliques}

\section{Vitesses moyennes de l'écoulement}

La vitesse d'un courant peut être rattachée à la pente de la ligne d'eau " $i$ " et au rayon hydraulique. " $R_{H}$ " par l'expression :

$$
\bar{U}=K R_{H}^{2 / 3} i^{1 / 2}
$$

$\vec{U}$ étant la vitesse moyenne du fluide, $K$ le coefficient de Strickler (voisin de 100 pour un sol parfaitement lisse) et $R_{H}$ le rayon hydraulique, c'est-à-dire le rapport 
entre la surface mouillée par le périmètre mouillé (que l'on peut assimiler dans certains cas à la profondeur d'eau).

Plus simplement on peut définir la vitesse moyenne comme étant le rapport entre le débit liquide $Q$ et la section transversale $S$ :

$$
\bar{U}=Q / S
$$

Ce paramètre ne permet pas d'avoir une très grande précision pour définir la vitesse critique d'entrainement d'un grain $\vec{U}$ puisque la vitesse critique d'entraînement dépend de la hauteur d'eau $H$ au-dessus du matériau.

$$
\vec{U}_{c}=f H^{1 / 6} \quad \begin{gathered}
\text { (en admettant une répartition } \\
\text { logarithmique de vitesses) }
\end{gathered}
$$

Par exemple si $\bar{U}_{c}=25 \mathrm{~cm} / \mathrm{s}$ pour une hauteur d'eau $I I$ de $0,10 \mathrm{~m}, \bar{U}_{c}$ sera égal à $36 \mathrm{~cm} / \mathrm{s}$ pour une hauteur d'eau de $1 \mathrm{~m}$ et à $55 \mathrm{~cm} / \mathrm{s}$ pour une hauteur de $10 \mathrm{~m}$.

\section{Force tractrice s'exerçant sur le fond}

Aussi préfère-t-on caractériser le début d'entraînement d'un grain par la force tractrice $\tau_{o}$ s'exerçant sur les fonds :

$$
\tau_{0}=\rho \cdot g \cdot R_{H} \cdot i
$$

avec:

$$
\begin{aligned}
& \rho=\text { masse spécifique de l'eau }\left(\mathrm{en} \mathrm{kg} / \mathrm{m}^{3}\right) \\
& g=\text { accélération de la pesanteur }(\mathrm{en} \mathrm{m} / \mathrm{s}) \\
& R_{H}=\text { rayon hydraulique (ou hauteur) en m } \\
& i=\text { pente de la ligne d'eau }
\end{aligned}
$$

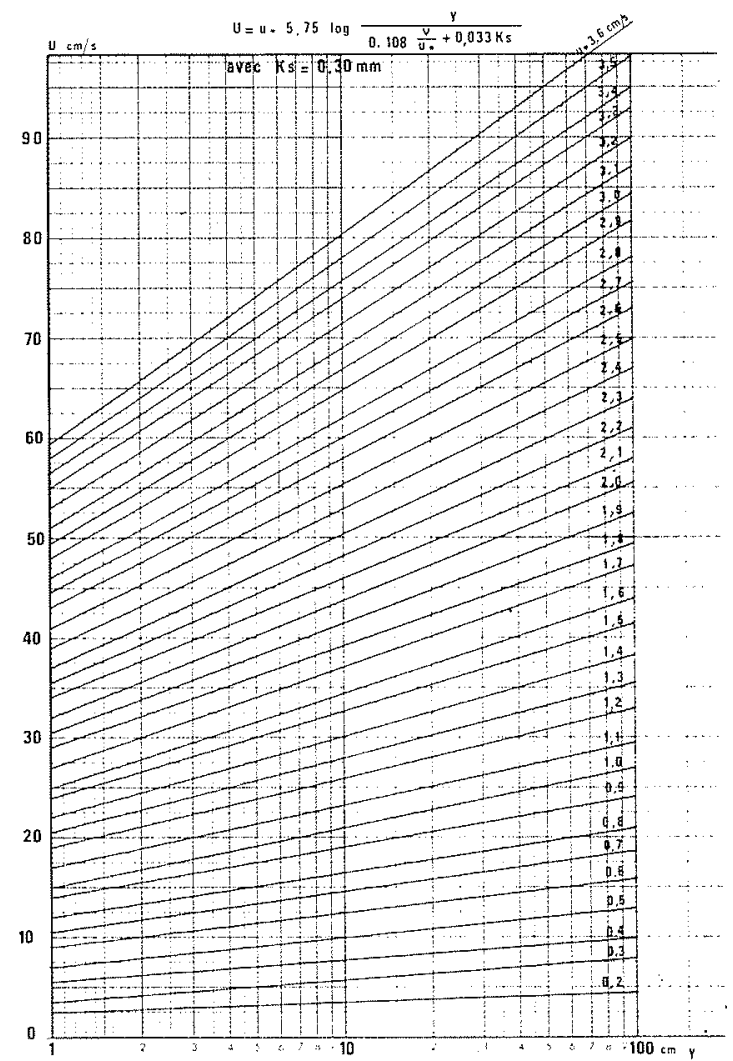

Par exemple, si l'on a dans un canal assez large une profondeur d'eau $H=1 \mathrm{~m}$ (voisine de la valeur de $R_{H}$ ), une pente de la ligne d'eau de 1 pour 1000 , on aura une valeur de la force tractrice de :

$$
\begin{aligned}
& \tau_{0}=1000 \mathrm{~kg} / \mathrm{m}^{3} \times 9,81 \mathrm{~m} / \mathrm{s} \times 1 \mathrm{~m} \times \frac{1}{1000} \\
& \tau_{0}=9,81 \mathrm{~N} / \mathrm{m}^{2} \text { ou } 1,0 \mathrm{kgf} / \mathrm{m}^{2}
\end{aligned}
$$

\section{Vitesse de frottement sur le fond}

De même, il est possible de rattacher le début d'entraînement d'une particule à la force de frottement " $u$ " que l'on peut déduire directement des mesures de vitesses au-dessus des fonds (Figures 15 et 16)

$$
U / u_{*}=5,75 \log y / 0,108 \frac{\nu}{u_{*}}+0,033 k_{s}
$$

$U$ étant la vitesse mesurée à une distance $y$ du fond, $k_{s}$ le coefficient de rugosité de Nikuradse et $\nu$ la viscosité de l'eau. Cette formule est valable pour toute la tranche d'eau $y$ située à moins de $0,16 H$ du fond ( $H$ étant la profondeur totale).

Si le coefficient $k_{s}$ est négligeable (cas de fonds lisses) et l'eau à une température de $15^{\circ} \mathrm{C}$

$\left(\nu=1,15 \cdot 10^{-2} \mathrm{~cm}^{2} / \mathrm{s}\right)$

on aura :

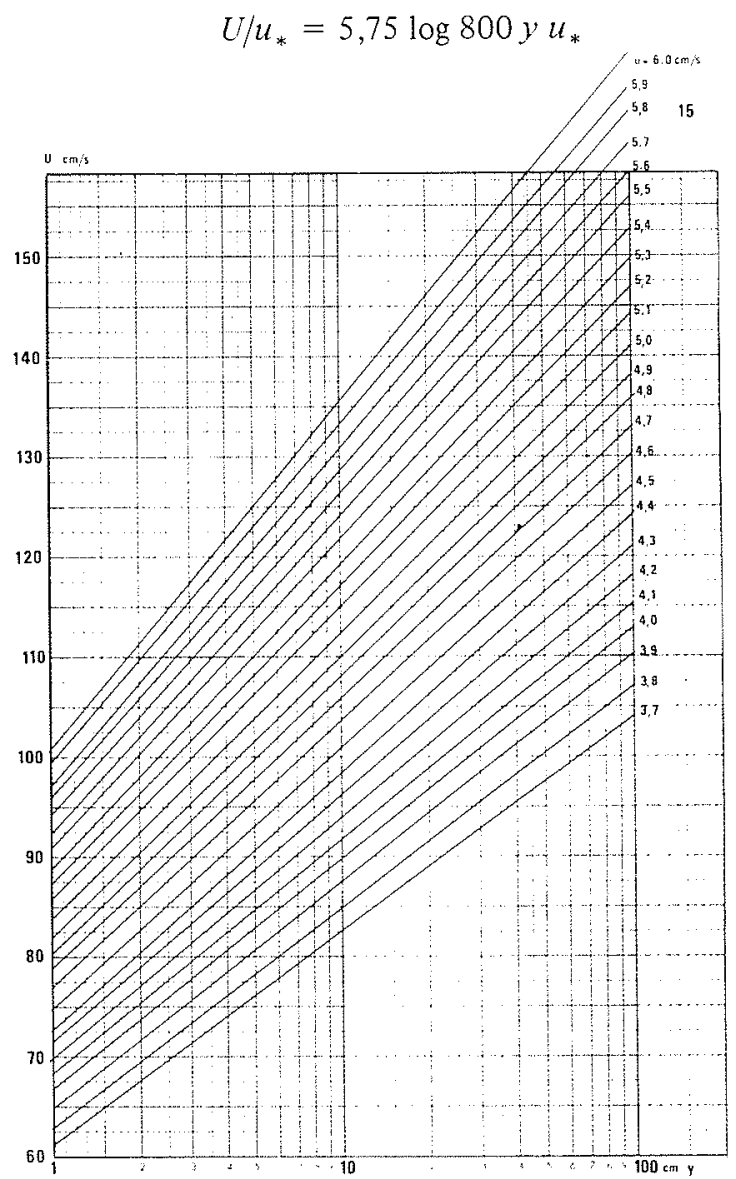

Figure 15 - Valeurs de $u_{*}$ pour $K_{s}=0,3 \mathrm{~mm}$ 


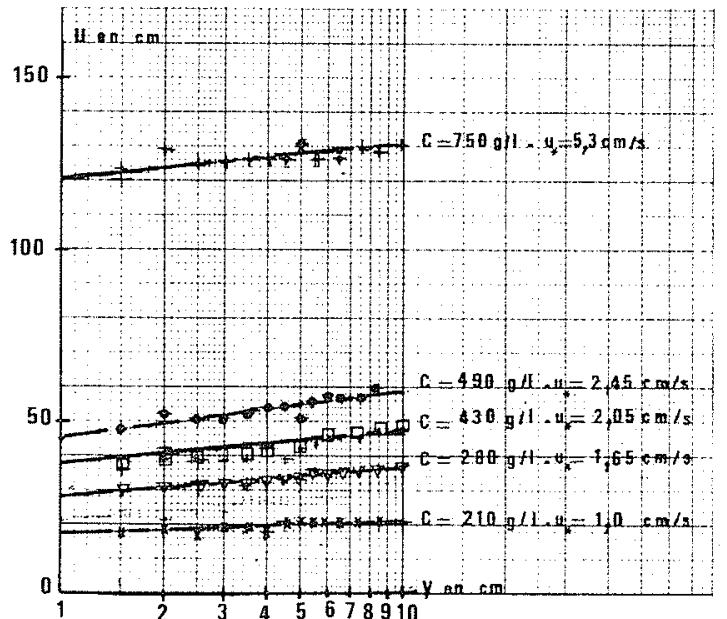

Figure 16 - Exemple de la répartition des vitesses sur le fond Vitesses critiques de reprise pour différentes concentrations de vases

A titre d'exemple si $u_{*}=1 \mathrm{~cm} / \mathrm{s}$, on devrait avoir $U=16,6 \mathrm{~cm} / \mathrm{s}$ à $1 \mathrm{~cm}$ du fond et $22,5 \mathrm{~cm} / \mathrm{s}$ à $10 \mathrm{~cm} \mathrm{du}$ fond.

\section{Relations entre vitesse de frottement, force tractrice et vitesse moyenne}

La vitesse de frottement $u_{*}$ est rattachée à la force tractrice par l'expression :

$$
u_{*}=\left(\tau_{0} / \rho\right)^{1 / 2} \text { et } u_{*}=\left(g \cdot R_{H} \cdot i\right)^{1 / 2}
$$

Cette vitesse de frottement $u_{*}$ peut également être rattachée en régime uniforme à la vitesse moyenne $\bar{U}$ par le coefficient $C$ de Chezy :

$$
\bar{U} / u_{*}=C / g^{1 / 2}=K R_{H}^{1 / 6} / g^{1 / 2}
$$

Ces quelques expressions de $\bar{U}, \tau_{0}, u_{*}$ permettent de caractériser l'écoulement et de définir à partir soit des mesures de pente de la ligne d'eau, soit de la répartition des vitesses au-dessus des fonds, soit des mesures du débit, une valeur "hydraulique" définissant la mise en mouvement d'une particule solide.

\section{Début d'entrainement des sédiments par un courant}

\section{Matériaux grossiers non cohésifs}

\section{Force tractrice de début d'entraînement}

Les particules sédimentaires sont caractérisées par leur diamètre, leur densité, leur forme et leur état de surface.

Les différentes expériences effectuées en laboratoire sur le début d'entrainement des matériaux montrent que la force tractrice critique nécessaire pour remettre une particule en mouvement est proportionnelle, d'une part au diamètre du grain $d$, d'autre part à sa densité relative (densité sous l'eau).

$$
\tau_{c}=A\left(\gamma_{s}-\gamma_{o}\right) d
$$

ou

$$
\tau_{c}=A\left(\rho_{s}-\rho_{o}\right) d g
$$

avec :

$\gamma_{s}$ et $\gamma_{o}$ poids volumiques du matériau et du fluide $(\gamma=\rho g)$

$A$ un paramètre compris entre 0,04 et 0,06 (moyenne $=0,05$ )

$d$ le diamètre du grain (diamètre moyen)

Pour un sable de masse spécifique $\rho=2600 \mathrm{~kg} / \mathrm{m}^{3}$, la force tractrice sera voisine de :

$$
\tau_{c}=0,8 d \dot{\mathrm{a}} 1,0 d
$$

si $\tau_{c}$ est en Newton par $\mathrm{m}^{2}$ et $d$ en $\mathrm{mm}$.

ou de : $\tau_{c}=0,08 d$ à $0,10 d$

si $\gamma_{c}$ est en $\mathrm{kgf}$ par $\mathrm{m}^{2}$ et $d$ en $\mathrm{mm}$.

Rappelons que $1 \mathrm{~N} / \mathrm{m}^{2}=0,1 \mathrm{kgf} / \mathrm{m}^{2}$.

A titre d'exemple, si la force tractrice critique est supérieure à $1 \mathrm{~N} / \mathrm{m}^{2}$ (ou à $0,1 \mathrm{kgf} / \mathrm{m}^{2}$ ), les particules inférieures à $1 \mathrm{~mm}$ seront mises en mouvement. Il faudra $10 \mathrm{~N} / \mathrm{m}^{2}$ pour arracher un gravier de $1 \mathrm{~cm}$. (Figure 17).

En fait la valeur exacte de la force tractice de début d'entraînement dépend du critère que l'on s'est fixé pour caractériser le mouvement. Suivant que l'on prend comme définition le départ d'un grain, de quelques grains ou un mouvement généralisé les valeurs peuvent passer de 1 à 4 .

De même il est maintenant prouvé que l'on ne peut extrapoler les résultats aux sables fins en admettant une fonction linéaire entre la force tractrice et le diamètre des grains. A partir des particules de $0,3 \mathrm{~mm}$ de diamètre (ou méme $0,6 \mathrm{~mm}$ d'après les graphiques de l'US Corps of Engineers) la force tractrice atteindrait une valeur minimale comme l'illustrent les deux figures ci-après (figures 18 et 19 ).

Relation entre la force tractrice critique et la vitesse moyenne d'érosion

Connaissant la force tractrice on peut en déduire la vitesse de frottement $u_{*}$ et la vitesse moyenne $\bar{U}$ nécessaire pour déplacer une particule.

En admettant une répartition logarithmique des vitesses au-dessus des fonds on peut préciser la vitesse moyenne d'entraînement d'une particule déterminée pour différentes hauteurs d'eau (figure 20).

Par exemple pour remettre en mouvement une particule de $2 \mathrm{~mm}$ par une profondeur d'eau de $10 \mathrm{~m}$, il faudra une vitesse moyenne du courant de $0,8 \mathrm{~m} / \mathrm{s}$, alors que dans $10 \mathrm{~cm}$ d'eau il suffirait d'une vitesse de $0,35 \mathrm{~m} / \mathrm{s}$.

En fait, on constate qu'en mer la répartition des vitesses dues aux courants de marée ne suit pas exactement cette loi logarithmique et que les particules se mettront en mouvement pour des valeurs de 


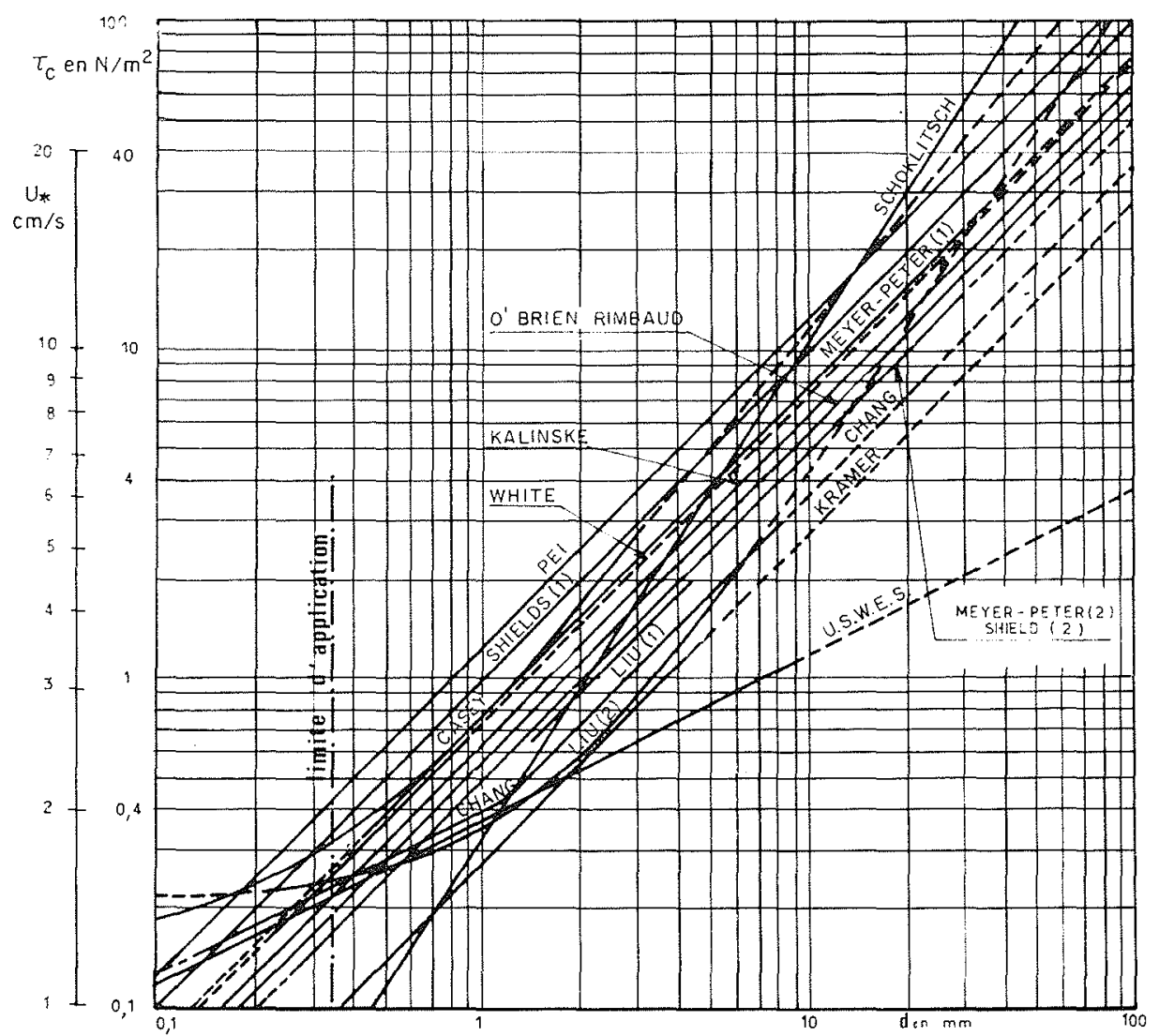

Figure 17 - Force tractrice et vitesse de frottement nécessaires pour entrainer une particule solide

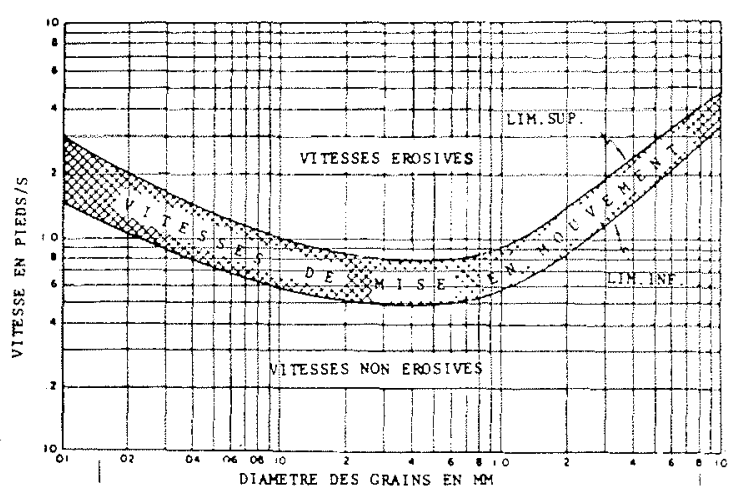

Figure 18 - Influence du diamètre des petites particules sur la vitesse critique de reprise

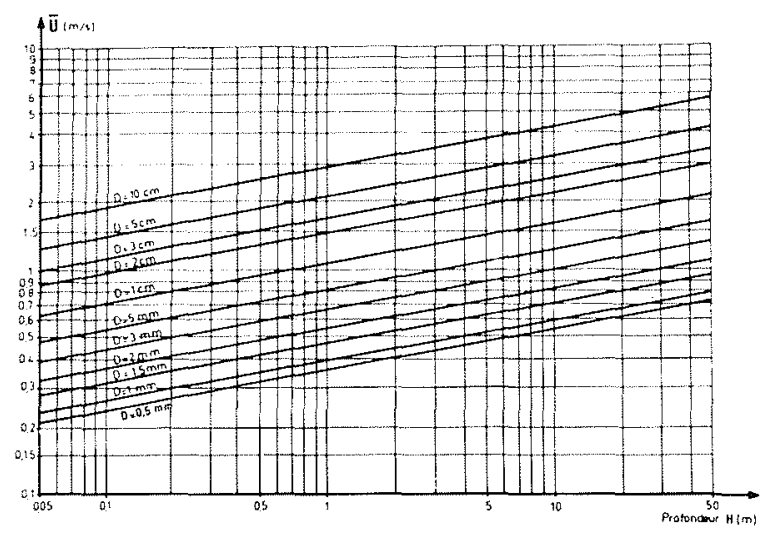

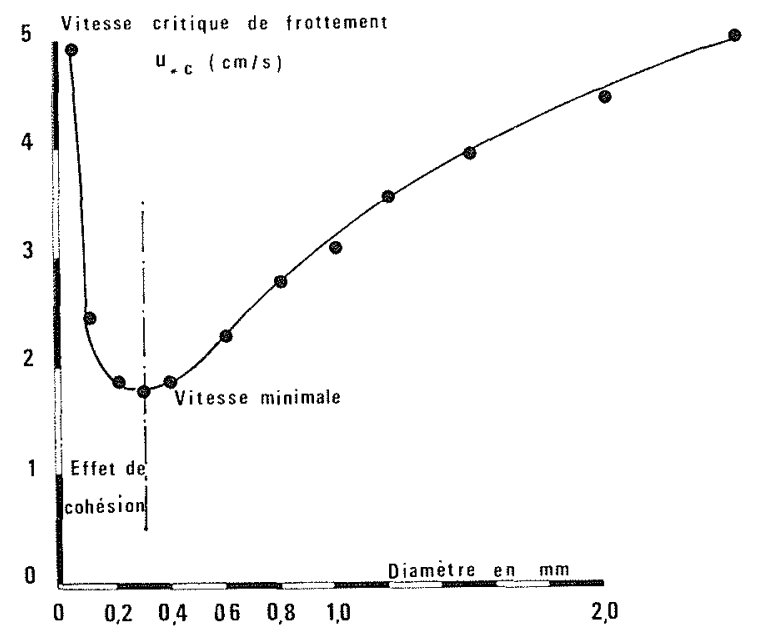

Figure 19 - Vitesse critique de frottement nécessairc pour provoquer l'entraìnement des sables de 0,1 à $2,0 \mathrm{~mm}$ 
vitesses sensiblement plus faibles que celles qui pourraient être calculées en supposant les profondeurs supérieures à $10 \mathrm{~m}$.

Paramètres sans dimension permettant de aéfinir le début d'entraînement des particules

J. Valembois a montré (Coastal Engineering, Aout 1960) qu'il était possible de représenter le début d'oscillation de particules sous l'action de la houle en introduisant un nombre sans dimension $G$ dont l'importance pour le transport solide est considérable. Ce nombre sans dimension est le rapport de $\tau_{o} / \Sigma \rho g d$ à $R_{*}^{2}$, et s'écrit :

$$
G=\frac{\sum g d^{3}}{\nu^{2}} .
$$

$\Sigma=$ densité relative du matériau.

Ce nombre $G$ est appelé "paramètre du grain" que l'on retrouvera dans de nombreuses formules de transport. Ce paramètre caractérise le comportement des grains dans les fluides et l'on peut écrire :

$$
G=f\left(R_{*}\right)
$$

$R_{*}=u_{*} d / \nu$ étant le nombre de Reynolds étoilé rapporté au grain.

Partant de la théorie de la couche limite laminaire J. Valembois a, par ailleurs, calcule la tension tangen- tielle $\tau_{0}$ sur les fonds soumis à la houle

$$
\tau_{0}=\nu \rho \zeta_{\max } / \sqrt{\nu \tau}
$$

et présenté le début d'entrainement des particules dans la houle en fonction des paramètres sans dimension $R_{*}^{2}$ et $G$. Il a montré à cette époque que les points expérimentaux se groupaient suivant une droite pour $R_{*}^{2}$ compris entre 1 et $3.10^{2}$, l'équation de cette droite étant :

$$
G=10 R_{\%}^{8 / 3}
$$

Sous une forme identique, Bonnefille a présenté un ensemble de résultats expérimentaux obtenus par différents laboratoires en fonction des paramètres

$$
d_{*}=G^{1 / 3}=\left(\Sigma g / \nu^{2}\right)^{1 / 3} d \quad \text { et } R_{*}=u_{*} d / \nu
$$

En faisant une analyse statistique on constate qu'il existe, comme J. Valembois l'avait montré, une relation linéaire entre $d_{*}$ et $f\left(R_{*}\right)$

$$
\begin{array}{r}
\text { Pour } 0,3<R e_{*}<12 \text { on a } d_{*}=2,5 R e_{*}^{4 / 5} \\
R e_{*}>12 \text { on a } d_{*}=3,8 R e_{*}^{5 / 8}
\end{array}
$$

La valeur de $R e^{*}=12$, pour laquelle apparaît une discontinuité expérimentale, correspond au cas où le diamètre du sédiment du fond est sensiblement égal à l'épaisseur de la couche limite laminaire " $e$ " qui,

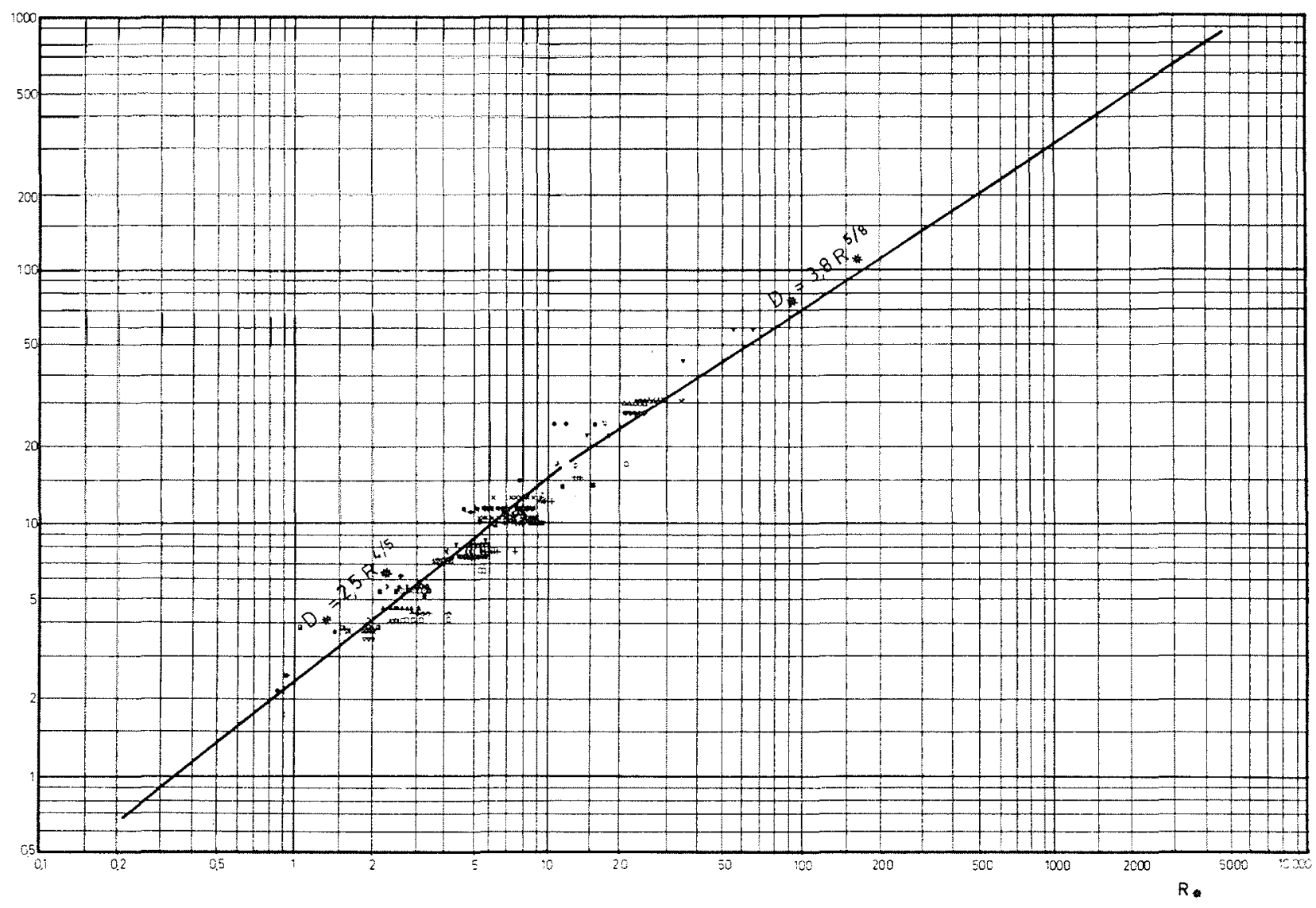

Figure 21 - Loi de début d'entraînement des sédiments 
selon la théorie de Prandtl, est définie par le nombre de Reynolds :

$$
u_{*} e / \nu=11,6
$$

Par exemple soit un cours d'eau de profondeur $H=1 \mathrm{~m}$, de pente $i=1 / 1000$ sur un fond de sédiments de diamètre $1 \mathrm{~mm}$.

La vitesse de frottement est égale à :

$$
u_{*}=(g H i)^{1 / 2}=10 \mathrm{~cm} / \mathrm{s}
$$

Le nombre de Reynolds correspondant est :

$$
R e_{*}=100
$$

Le diamètre étoilé correspondant est : $d_{*}=65$. On en déduit le diamètre des matériaux susceptibles d'être érodés à partir de la formule :

$$
d_{*}=\left(\epsilon g / \nu^{2}\right)^{1 / 3} d
$$

$d=2,5 \cdot 10^{-3}=2,5 \mathrm{~mm}$. Les matériaux inférieurs à cette valeur seront érodés, donc les particules de $1 \mathrm{~mm}$ de diamètre.

Si au lieu de sédiments de $1 \mathrm{~mm}$ on avait pris un fond de graviers de $1 \mathrm{~cm}$, les mêmes calculs donneraient :

$$
R e_{*}=1000, d_{*}=180 \text { d'où } d=7 \mathrm{~mm}
$$

Les graviers de $1 \mathrm{~cm}$ de diamètre ne seront pas érodés.

\section{Matériaux cohésifs (limons - vases - argiles)}

Pour les sédiments cohésifs du type vases - limons ou argiles on constate que la vitesse de frottement, ou la force tractrice nécessaire pour éroder le dépôt, varie considérablement suivant son état de tassement et la nature du complexe sol-eau. Le diamètre et la densité des particules élémentaires ne sont plus suffisants pour déterminer le début d'érosion et il faut tenir compte de la rigidité initiale du dépôt.

Des vases fraichement déposées, ou des vases contenant des ions très hydratés, se comporteront comme un fluide dense et l'on constatera à l'interface de l'eau et de la vase la formation d'ondes qui pourront littéralement déferler en remettant une grosse partie des dépôts en suspension.

Des vases, ayant subi un début de consolidation et prenant un aspect plastique au contraire, offriront une certaine résistance au courant et l'on observera, au début de l'action du fluide des figures d'étirement du dépôt avec arrachement de copeaux puis "explosion" du sol.

Après un stade de tassement prolongé les dépôts cohésifs présenteront une très grande résistance à l'érosion et seuls des copeaux et des blocs de vase pourront être entraînés pour les très grandes vitesses.

Les nombreuses études faites au L.C.H.F. (Houille Blanche 1968) ont montré que l'on pouvait, pour les vases ayant subi un début de consolidation, rattacher la vitesse critique d'érosion $u_{*}$ à la rigidité initiale du dépôt $\tau y$ par une relation de la forme :

$$
u_{*_{c}}=K \tau y^{1 / 2}
$$

si $u_{*}$ est exprimé en $\mathrm{m} / \mathrm{s}$ et $\tau y$ en $N / \mathrm{m}^{2}$ le coefficient $K$ est égal à 0,030 pour un entraînement généralisé et à 0,016 pour le début d'entraînement (figures $22-23$ et 24).

\section{Débits solides transportés par les courants}

\section{Généralités}

Arrachées au fond, les particules solides vont être transportées en charriage ou en suspension. Suivant l'importance de l'écoulement les quantités transportées pourront varier entre quelques milliers de $\mathrm{m}^{3}$ et plusieurs millions de $\mathrm{m}^{3}$ par an. Certains torrents ou oueds, dans des régions semi-arides, pourront transporter en quelques jours 5 à 20 millions de tonnes de matériaux comme par exemple le Sefid-Roud en Iran, l'Oued Fodda et l'Isser en Algérie. Comparativement à nos grandes rivières comme la Loire, la Seine ou la Garonne, ces valeurs sont importantes puisque, pour ces dernières on ne retrouve à leur partie aval que 1 à 3 millions de tonnes par an de sédiments dont la plus grande partie se trouve en suspension dans les eaux.

Les transports en suspension sont relativement faciles à mesurer et il suffit de connaitre les vitesses et les turbidités des eaux, c'est-à-dire la concentration en particules solides en un point donné, pour en déduire avec une bonne approximation l'ordre de grandeur des quantités déplacées en suspension. Dans certaines rivières les turbidités des eaux peuvent atteindre des valeurs considérables et l'on a pu mesurer des concentrations en particules dépassant 200 grammes par litre d'eau (Oued ISSER en forte crue). Dans ces suspensions on trouve évidemment une majorité d'éléments très fins du type argile - boue - vase et silts mais si la turbulence est importante il peut s'y ajouter des éléments beaucoup plus gros tels que les sables et les graviers qui restent d'autant mieux en suspension que la viscosité de l'eau se trouve modifiée par la suspension.

Les transports par charriage sont beaucoup plus difficiles à apprécier et les techniques des "pièges à sable", que l'on dispose sur les fonds, donnent des résultats assez dispersés. L'utilisation de traceurs radioactifs permet de mieux approcher le problème mais cette technique nécessite des spécialistes confirmés et l'interprétation des résultats reste délicate. On peut évidemment obtenir des résultats valables lorsque l'on dispose de zones de décantation sur le cours d'eau, telles qu'une zone d'emprunt de sables et graviers par dragage dans une rivière où la création d'un barrage ; il s'agit là d'une évaluation globale du transport.

De même, à partir de l'évaluation du transport en suspension, on peut apprécier pour certaines catégories de rivière l'ordre de grandeur du charriage. Ce dernier serait d'environ 10 à $15 \%$ du transport en suspension.

Pour palier à ces difficultés de mesure, des efforts importants ont été réalisés pour essayer à l'aide de formules de charriage de dégager des ordres de grandeur 


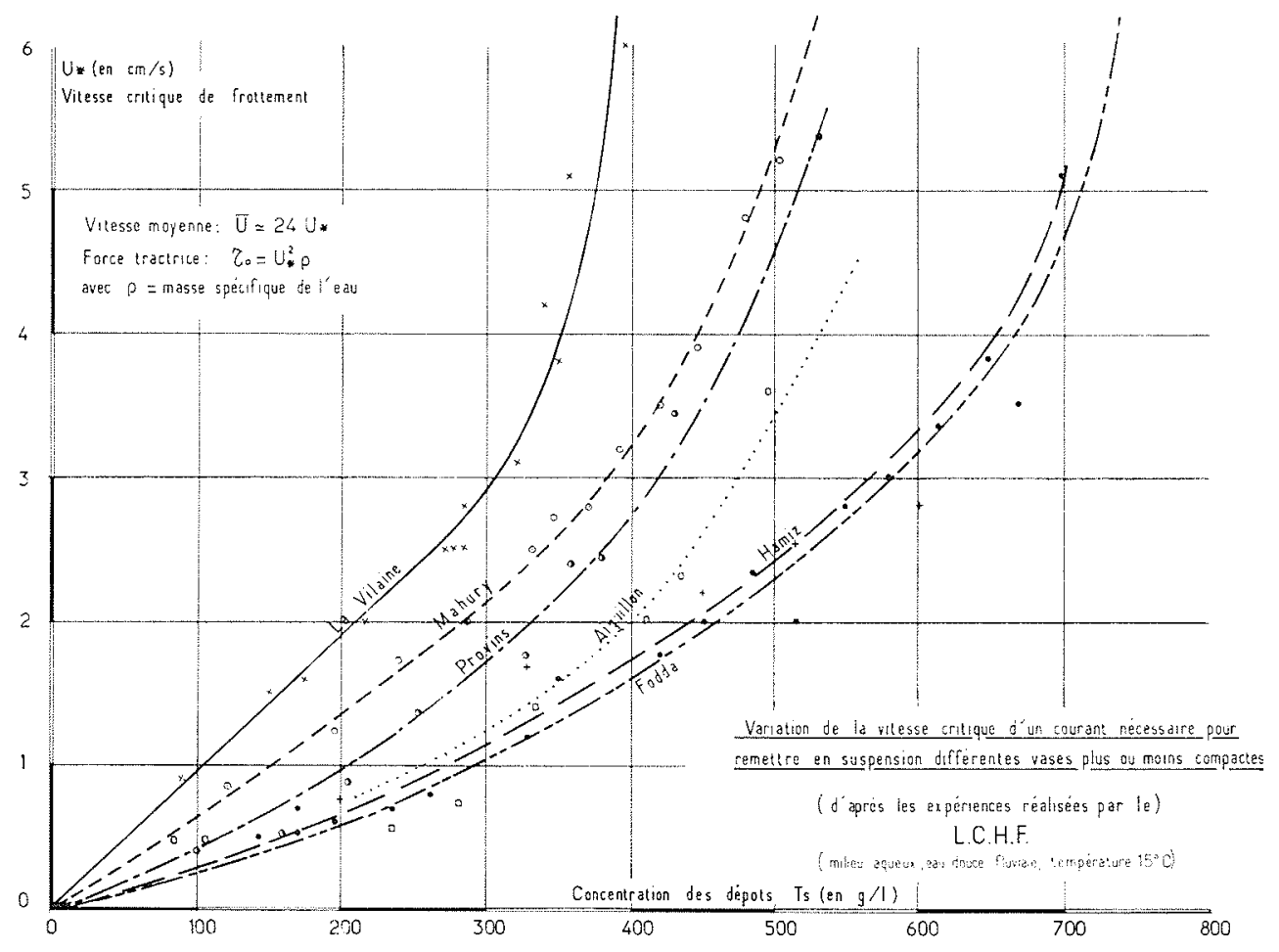

Figure 22 - Influence de la concentration en particules solides et de la nature des sédiments

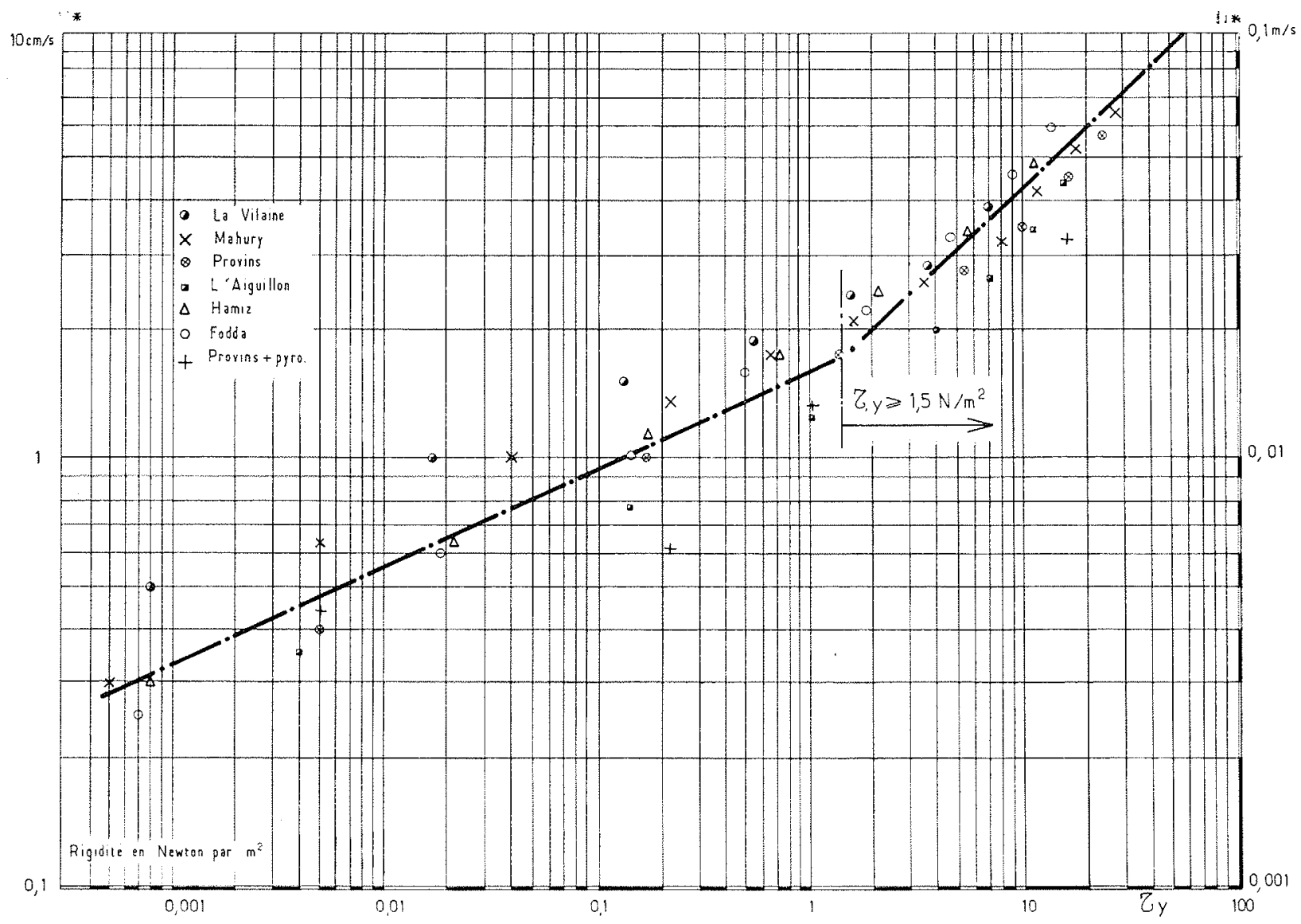

Figure 23 - Relation entre la vitesse critique de frottement $u_{*}$ et la rigidité initiale $\tau y$ 


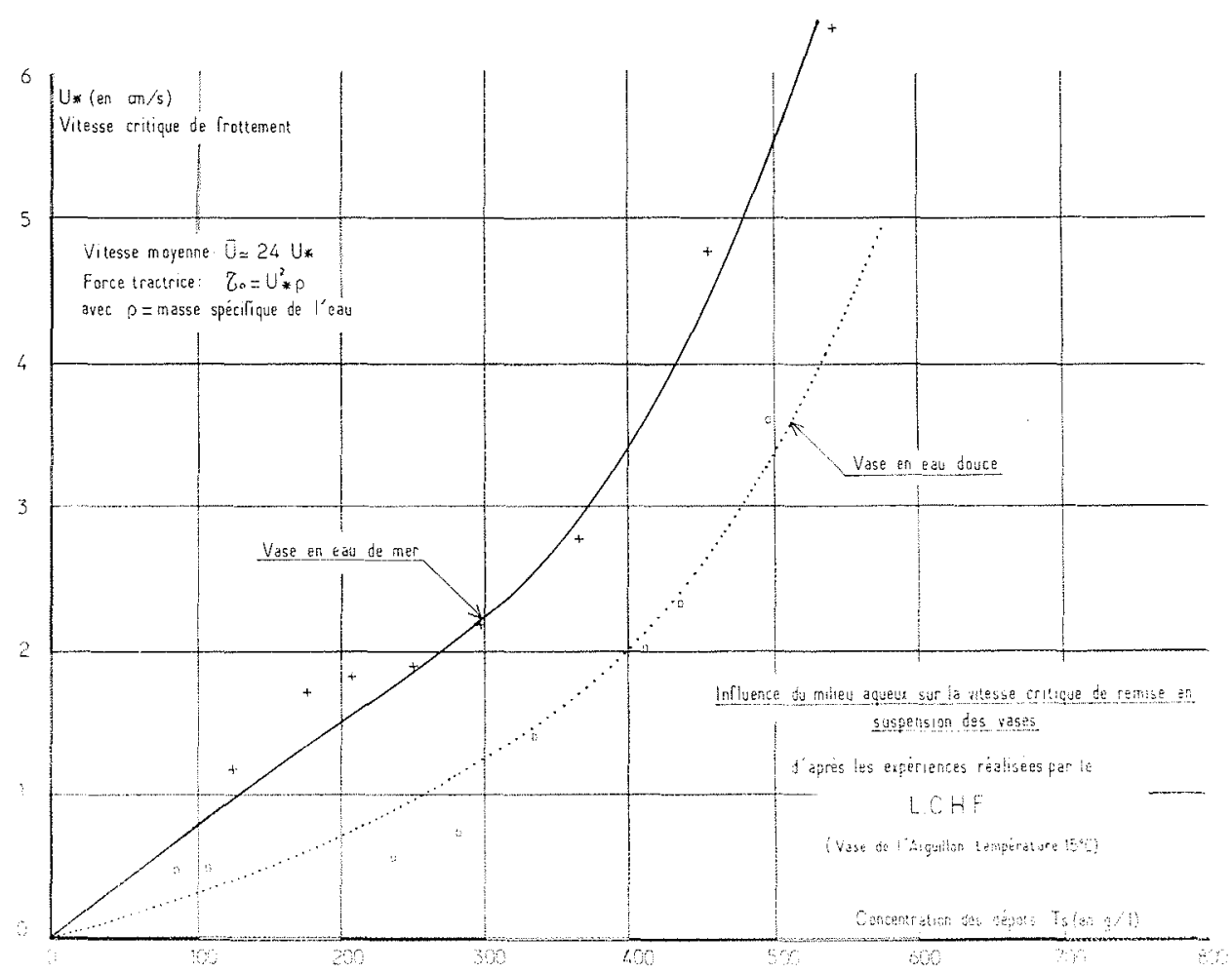

Figure 24 - Influence du milicu aqueux sur l'érosion des vases

du transport solide. Ces formules sont nombreuses, donnent en général des résultats assez différents les uns des autres et il faut les utiliser avec prudence en essayant de dégager les limites d'utilisation.

\section{Débit solide des sables, graviers et galets}

Les formules de transport par charriage et saltation peuvent être basées sur le concept de force tractrice et de vitesse moyenne ou sur le concept de turbulence.

\section{Formules basées sur le concept de Force Tractrice}

Parmi les formules basées sur le concept de vitesse moyenne et de force tractrice on trouve l'ancienne for. mule de Duboys (1879) selon laquelle on admet que le transport s'effectue dans une série de couches superposées, d'une épaisseur approximativement égale au diamètre d'une particule, et que la vitesse des couches superficielles décroît linéairement (d'une quantité $\Delta u_{s}$ ) depuis un maximum pour la couche superficielle jusqu'à zéro pour la nième couche. On arrive à une formule de la forme:

$$
g_{s}=\Psi^{\prime} \tau_{0}\left(\tau_{0}-\tau_{c}\right)
$$

$g_{s}$ étant le débit solide en poids par mètre de largeur,

$\tau_{0}$ ia force tractrice sur le fond exercée par le fluide,

$\tau_{c}$ la force tractrice critjque de début d'entraînement des particules,

$\Psi^{\prime}$ un coefficient qui dépend d'un certain nombre de paramètres.

De très nombreuses formules sont de la forme de celle de Duboys et l'on peut citer par exemple les for- mules de Mac Dougall (1933), Schoklitsch (1934), Chang, Uswes (1935), Schields (1936), Meyer Peter et Muller (1948).

La formule de Meyer Peter présente l'avantage de faire intervenir une correction de parois et une distinction entre la rugosité due aux berges et aux grains. En la simplifiant on peut la mettre sous la forme :

$$
\begin{aligned}
g_{s} & =A\left(\tau_{0}-\tau_{c}\right)^{3 / 2} \\
\text { avec : } \quad A & =8\left(\frac{g}{\gamma_{0}}\right)^{1 / 2} \frac{\gamma_{s}}{\gamma_{s}-\gamma_{0}}
\end{aligned}
$$

$A=1,29$ en M.Kp.s et 0,40 en S.I,

$g_{s}$ est le débit solide en poids par mètre de largeur.

Supposons une profondeur d'eau de $1 \mathrm{~m}=H$, une pente $i$ de $5,5 \cdot 10^{-3}$ et un poids volumique

$$
\gamma=1000 \mathrm{kgf} / \mathrm{m}^{3} \text {. }
$$

on aura :

$$
\begin{aligned}
\tau_{0} & =\gamma_{H i} \\
& =5,5 \mathrm{kgf} / \mathrm{m}^{2}
\end{aligned}
$$

Si l'on considère un galet ayant une vitesse critique d'entrainement, ou une force tractrice critique d'entrainement de $4,5 \mathrm{kgf} / \mathrm{m}^{2}$ (galets de $5 \mathrm{~cm}$ de diamètre)

on aura :

$$
\begin{aligned}
& g_{s}=1,29(5,5-4,5)^{3 / 2} \\
& g_{s}=1,29 \mathrm{~kg} / \mathrm{m} / \mathrm{s}
\end{aligned}
$$

Pour une rivière large de $10 \mathrm{~m}$, le débit solide quotidien sera de $1100 \mathrm{t} /$ jour et pour une moyenne de 100 
jours par an le débit solide total annuel peut être évalué à $110000 \mathrm{t} / \mathrm{an}$, ce qui représente le débit solide d'un torrent alpin ayant un bassin versant de $200 \mathrm{~km}^{2}$ par exemple.

\section{Formules basées sur le concept de la turbulence}

Les équations de Duboys et Meyer Peter admettaient que le cisaillement agissant sur les particules du lit était constant et égal à sa valeur moyenne. Pourtant les forces du fluide qui agissent subissent des fluctuations en fonction du temps par suite de la turbulence du fluide.

Kalinske a, le premier, cherché à évaluer l'effet des fluctuations dues à la turbulence sur la vitesse du fluide près des particules et par conséquent sur les forces exercées par le fluide.

Einstein, en se fondant sur l'expérience, est arrivé à la conclusion que les particules sont transportées le long du lit suivant une série d'étapes dont la longueur est, en moyenne, proportionnelle à la taille de la particule et que celle-ci se dépose sur le fond après avoir parcouru une ou plusieurs de ces étapes. Le taux de dépôt par unité de surface dépend alors du taux de transport à travers une section donnée et de la probabilité pour que les forces hydrodynamiques soient telles que les particules puissent se déposer. D'autre part, le taux d'érosion de cette surface dépend du nombre et des propriétés des particules qui s'y trouvent et de la probabilité que la force hydrodynamique sur chaque particule soit suffisamment grande pour la déplacer. Pour que le lit soit stable le taux de la sédimentation doit être égal au taux de l'érosion.

En forme simplifiée, on a :

$$
\Phi^{\prime}=f(\psi)
$$

avec

$$
\begin{aligned}
\psi & =\frac{\gamma_{s}-\gamma}{\gamma} \cdot \frac{D}{R_{H \gamma}}=\frac{\left(\gamma_{s}-\gamma\right) D}{\tau_{0}} \\
\Phi^{\prime} & =\frac{g_{s}}{A \gamma_{s}}\left(\frac{\gamma}{\gamma_{s}-\gamma}\right)^{1 / 2}\left(\frac{1}{g D^{3}}\right)^{1 / 2}
\end{aligned}
$$

$A$ est égal approximativement à 0,82

Dans une forme plus élaborée Einstein fait intervenir des paramètres traduisant l'influence de la distribution granulométrique et aussi le fait que les gros grains protègent les petits dans une distribution non uniforme. Einstein introduit par ailleurs une correction sur le rayon hydraulique traduisant l'influence de la rugosité des berges.

M. Bellessort a mis les résultats sous forme d'abaques (figure 25) qui permettent, connaissant la vitesse de frottement $u_{: *}$ (ou la force tractrice $\tau_{0}$ puisque l'on peut rattacher $\tau_{0} \quad \grave{a} u_{*}$ ), de définir le débit transporté par unité de largeur et unité de temps.

Si l'on a par exemple une vitesse critique de frottement $u_{*}$ de $5 \mathrm{~cm} / \mathrm{s}$ - ce qui correspondrait à une vitesse moyenne dans un canal de $1,25 \mathrm{~m} / \mathrm{s}$ - on aurait pour un sable de $0,2 \mathrm{~mm}$ de diamètre un débit solide de $1,5 \mathrm{~kg} / \mathrm{ms}$.
Pour un $u_{*}$ de $3 \mathrm{~cm} / \mathrm{s}$ ce débit ne serait plus que de $0,05 \mathrm{~kg} / \mathrm{ms}$ et de $0,0015 \mathrm{~kg} / \mathrm{ms}$ pour un $u_{*}$ de $2 \mathrm{~cm} / \mathrm{s}$.

De la même façon on a pu définir des abaques donnant le débit en charriage et le débit en suspension dans les eaux pour différentes valeurs de la vitesse de frottement $u_{*}$ (Figure 26).

Pour une vitesse critique de frottement de $u_{*}=5 \mathrm{~cm} / \mathrm{s}$ par exemple et un sable fin de $0,2 \mathrm{~mm}$, on aura un rapport entre le débit par charriage et le débit total, de 0,1 autrement dit $90 \%$ des matériaux seront transportés en suspension.

Pour des particules de $0,5 \mathrm{~mm}$ au contraire le transport en suspension ne serait que de $45 \%$.

Notons l'influence du rayon hydraulique $R_{H}$ dans ces problèmes de transport en suspension et en charriage.

\section{Comparaison des différentes formules de transport}

Pour comparer les différentes formules de transport entre elles on peut, après les avoir simplifiées, les présenter en fonction des relations :

$$
Q_{s} /\left(\epsilon g d^{3}\right)^{1 / 2} \quad \text { et } \quad f=\tau_{0}\left(\gamma_{s}-\gamma_{0}\right) d
$$

$Q_{s}$ étant le débit solide en volume $Q_{s}=g_{s} / \gamma_{0}$

$\epsilon$ la densité relative $\frac{\rho_{s}-\rho_{0}}{\rho_{0}}=\frac{\gamma_{s}-\gamma_{0}}{\gamma_{0}}$

$d$ le diamètre des grains

$\tau_{0} \quad$ la force tractrice exercée par le fluide sur le fond

On obtient pour les différentes formules les équations comparatives suivantes :

$$
\begin{array}{lll}
\text { Schields } & : & Q_{s} /\left(\varepsilon g d^{3}\right)^{1 / 2}=10 C_{*}(f-0,076) \\
& & (f)^{3 / 2} \\
\text { Meyer Peter }: & Q_{s} /\left(\varepsilon g d^{3}\right)^{1 / 2}=8(f-0,047)^{3 / 2} \\
\text { Kalinske }: & Q_{s} /\left(\varepsilon g d^{3}\right)^{1 / 2}=10 f^{5 / 2} \\
\text { Bonnefille }: & Q_{s} /\left(\varepsilon g d^{3}\right)^{1 / 2}=5,5 f^{3 / 2} \\
& & \left(4,62 f^{1 / 2}-1\right)^{1,25} \\
\text { Einstein } \quad: & Q_{s} /\left(\varepsilon g d^{3}\right)^{1 / 2}=32,6 f^{3}
\end{array}
$$

En mettant ces équations sous une forme graphique (figure 27) on constate que pour une même valeur de la force tractrice et des caractéristiques du sédiment on peut obtenir des écarts entre les volumes transportés pouvant atteindre 10. Si l'on avait utilisé l'équation de Colby les écarts auraient été encore plus importants.

\section{Débit solide des sédiments très fins du type limons et vases}

Une vase en suspension s'écoule lorsque les conditions requises sont remplies, comme un fluide homogène. Pour cela il faut que les particules suivent parfaitement le mouvement de l'eau, ce qui implique, d'après la mécanique des suspensions :

$$
d u_{*} / \nu \leqslant 2\left(\rho / \rho_{s}\right)^{1 / 2}
$$




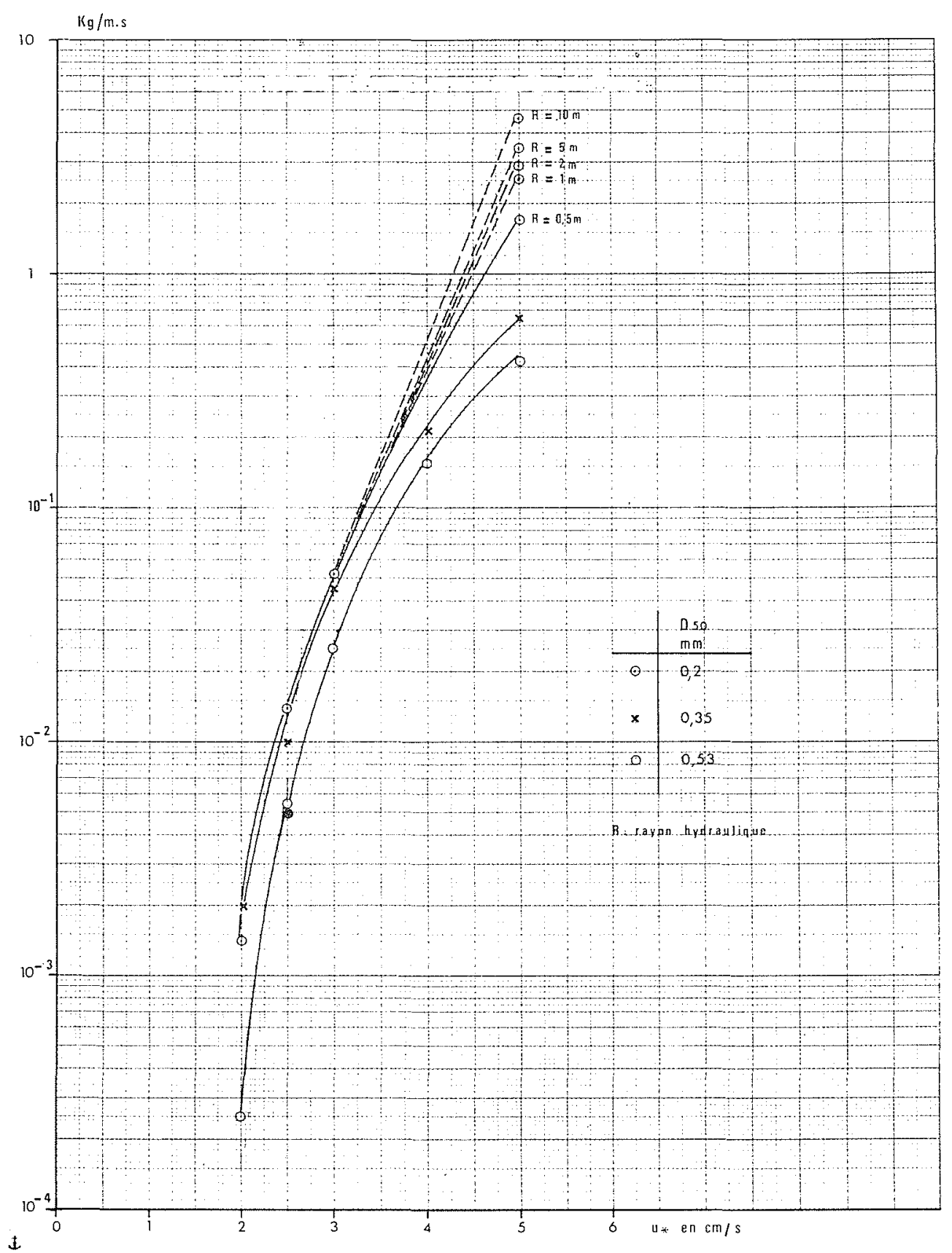

Figure 25 - Débit solide total $g_{S}$ en fonction de $u_{*}$

$d=$ diamètre de la particule, $u_{*}=\left(\tau_{0} / \rho\right)^{1 / 2}=$ vitessede frottement

$\rho=$ masse volumique de l'eau, $\rho_{s}=$ masse volumique des sédiments $\neq 2,6$

Si l'on prend comme ordre de grandeur

$$
u_{*}=0,10 \mathrm{~m} / \mathrm{s} \text { et } \nu=10^{-6} \mathrm{~m}^{3} / \mathrm{s}
$$

on obtient $d \leqslant 0,012 \mathrm{~mm}$, ce qui est le cas des vases.

Par ailleurs pour que la vase reste en suspension il faut que l'écoulement soit turbulent. M. Valembois a établi les équations pour que cette condition soit remplie en admettant que la suspension de vase répondait aux équations des fluides de Bingham (Houille Blanche $n^{\circ} 2 / 3-$ 1975).
Le débit minimum par unité de largeur d'une rivière " $Q_{\min }$ " doit être supérieur à une certaine valeur donnée parl'équation :

$$
Q_{\text {min }}=500 \nu \frac{(1+0,5 \alpha)}{(1-\alpha)^{2}}
$$

où :

$$
\alpha=\tau_{y} / \rho_{m} g h_{0} i
$$

avec :

$\nu=$ viscosité de la mixture en $\mathrm{m}^{2} / \mathrm{s}$,

$\tau_{y}=$ rigidité de la mixture en $N / \mathrm{m}^{2}$

$\rho_{m}=$ la densité de mixture en $\mathrm{kg} / \mathrm{m}^{3}$

On démontre, par ces équations, que les vases peuvent être transportées assez facilement par les courants et que 


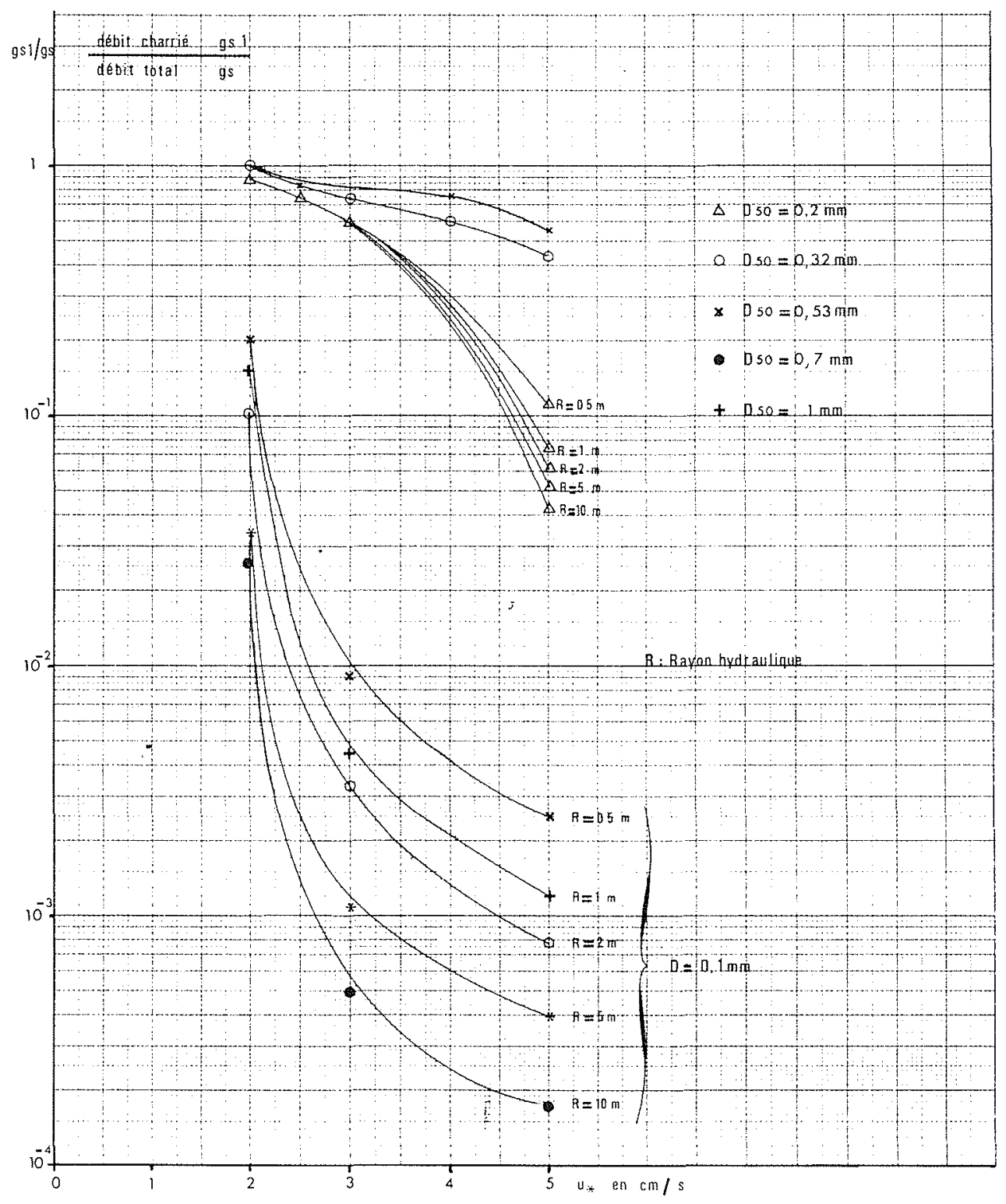

Figure 26 - Relation entre le débit total $g_{s}$ et le débit charrié $g_{S}$

l'on peut arriver dans un oued comme le HAMIZ en Algérie à transporter des concentrations atteignant $300 \mathrm{~g} / \mathrm{l}$ avec des débits de $20 \mathrm{~m}^{3} / \mathrm{s}$ pour une largeur du lit de $40 \mathrm{~m}$ et une pente inférieure à $1 \mathrm{~m}$ par kilomètre.

Dans une étude faite pour la Gironde, M. Cormault, partant de résultats obtenus au L.C.H.F. et complétés au L.N.H. (AIRH, Septembre 1971, Volume 4) admet que :

- la vitesse critique de reprise des vases de la Gironde croit selon la relation

$$
\begin{aligned}
& u_{*} \text { critique }=0,0055 T_{s}+0,0000026 T_{s}^{2} \\
& \text { avec } u_{*} \text { en } \mathrm{cm} / \mathrm{s} \text { et } T_{s} \text { en } \mathrm{g} / \mathrm{l}
\end{aligned}
$$

- le débit solide d'érosion dépend de la contrainte tangentielle $\tau_{0}$ exercée par l'écoulement sur ie fond et de la cohésion du fond intervenant dans u* critique. Ce débit solide d'érosion serait égal, dans le cas de la Gironde, à :

$$
\frac{a_{m}}{d_{t}}=2.10^{-5}\left[\left(\frac{u_{*}}{u_{*} \text { crit }}\right)^{2}-1\right] \mathrm{en} \mathrm{g} / \mathrm{cm}^{2} / \mathrm{s}
$$

\section{Comportement des sédiments sous l'action de la houle}

\section{Généralités}

Sous l'action de la houle, les sédiments déposés sur les fonds sont soumis à différentes forces : les unes provenant directement du mouvement orbitaire au voisinage du fond (figure 28), les autres des courants associés, c'est-à-dire des courants dans la couche limite et des courants de compensation (figure 29).

Près du littoral, la houle entraînera en plus un courant de houle parallèle à la côte dont le débit sera considérable et voisin, en tempête, de celui de nos grands fleuves en temps de crue.

Sur l'estran enfin les projections d'eau par les vagues engendreront des déplacements alternatifs des sédiments en "dents de scie" si les remises en suspension ne sont pas trop importantes. 


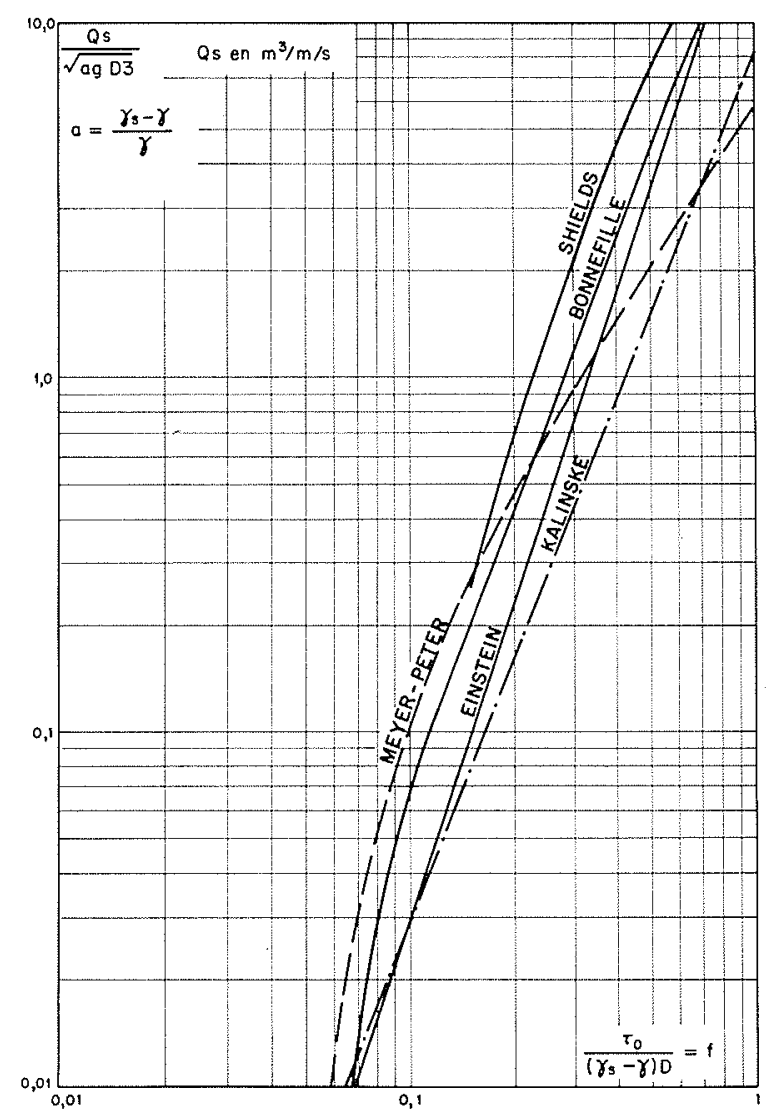

Figure 27 - Comparaison de quelques formules de transport

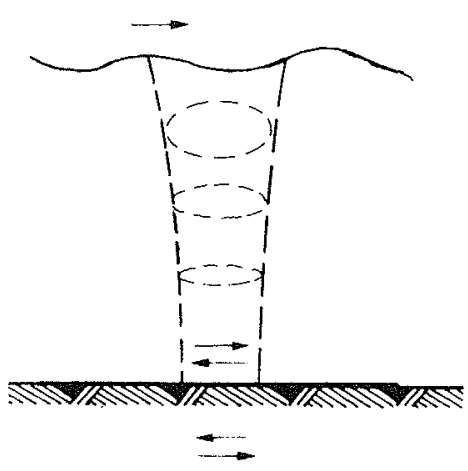

Figure 28 - Mouvements orbitaires

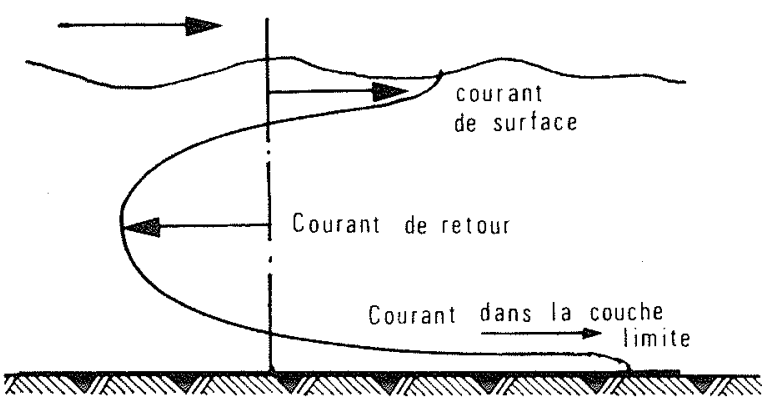

Figure 29 - Courants d'entraînement dans la masse du fluide

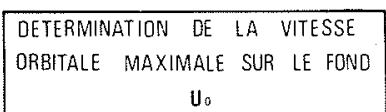

$$
U_{0}=\frac{\pi H}{T \operatorname{sh} 2 \pi \frac{d}{L}}
$$
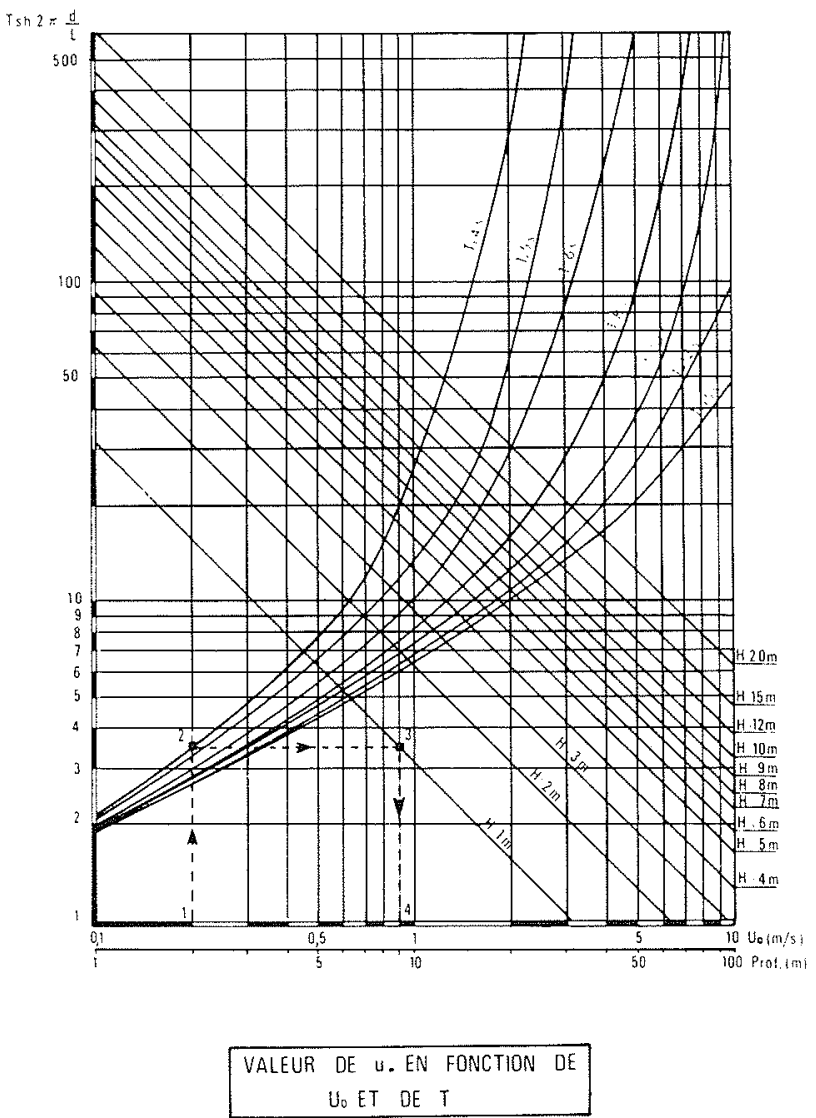

$$
u^{4}=\frac{8 Q U_{0}^{2}}{\pi T}
$$

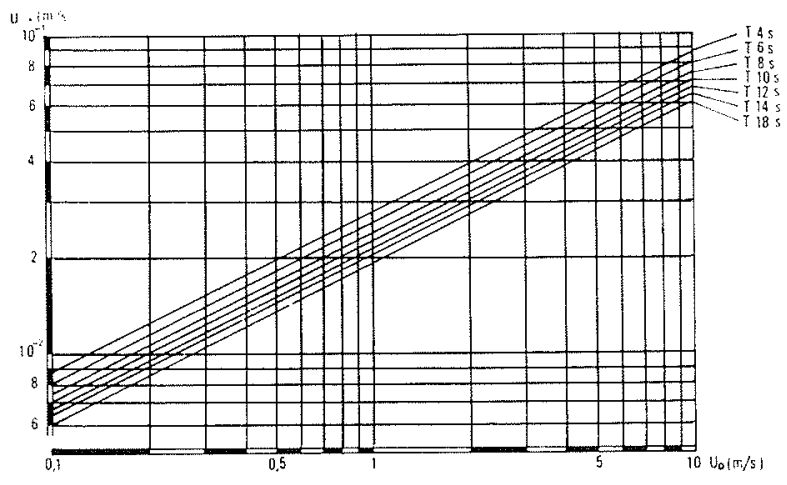

Figure 30 -Détermination de $U_{\max }$ ct $u$. 
Mouvements d'eau engendrés par la houle près du fond

\section{Vitesses alternatives sur les fonds}

Au voisinage $\mathrm{du}$ fond, la houle engendre des courants alternatifs susceptibles d'entraîner l'oscillation des particules solides si leur vitesse est supérieure à la vitesse critique d'entrainement des sédiments.

La vitesse orbitale maximale, $U_{\max }$, sur le fond est donnée par l'expression :

$$
U_{\max }=\frac{\pi h}{T \operatorname{sh} 2 \pi \frac{H}{L}}
$$

On peut d'autre part exprimer la vitesse de frottement $u_{*}$ sur le fond par:

$$
u_{*}=\left(\frac{8 \nu U_{\max }^{2}}{\pi T}\right)^{1 / 4}
$$

On en déduit :

$$
u_{*}=0,07\left(\frac{h^{2}}{T^{3} \operatorname{sh}^{2} 2 \pi \frac{H}{L}}\right)^{1 / 4}
$$

avec :

$\nu=$ viscosité de l'eau $=10^{-6} \mathrm{~m}^{2} / \mathrm{s}$ à $20^{\circ} \mathrm{C}$

$T=$ période de la houle en seconde $(s)$

$H=$ profondeur d'eau en mètre

$h=$ amplitude en mètre

$L=$ longueur d'ondes en mètre

On peut passer de la vitesse de frottement $u_{*}$ sur le fond à la force tractrice $\tau_{0}$ par l'expression :

$$
\tau_{0}=\rho u_{*}^{2}
$$

$\rho=$ masse spécifique de l'eau $=1000 \mathrm{~kg} / \mathrm{m}^{3}$

$\tau_{0}$ en Newton $/ \mathrm{m}^{2}$ si $u_{*}$ en $\mathrm{m} / \mathrm{s}$

Les abaques donnés fig. 30 permettent, connaissant la profondeur et les caractéristiques de la houle, de définir la valeur de $U_{\max }$ et de $u_{*}$,

Par exemple, si une houle de $10 \mathrm{~s}$ de période et $5 \mathrm{~m}$ d'amplitude est observée, elle donnera sur les fonds de $-10 \mathrm{~m}$ une vitesse maximale d'oscillation de :

$$
U_{\max }=2 \mathrm{~m} / \mathrm{s}
$$

et une vitesse de frottement $u_{*}$ de

$$
u_{*}=3 \cdot 10^{-2} \mathrm{~m} / \mathrm{s}=3 \mathrm{~cm} / \mathrm{s}
$$

La force tractrice $\tau_{0}$ sera de :

$$
\tau_{0}=1000 \mathrm{~kg} / \mathrm{m}^{3} \times\left(3.10^{-2}\right)^{2}=0,9 \mathrm{~N} / \mathrm{m}^{2}
$$

En appliquant ces résultats aux houles du Golfe de Gascogne on obtiendrait aux différentes profondeurs du plateau continental les valeurs suivantes de la vitesse de frottement $u_{*}$ en fonction de l'amplitude de la houle (figure 31). Dans le calcul on a admis qu'il existait une relation entre la période et l'amplitude telle que :

$$
T(\text { en } s)=h(\text { en } m)+8(\text { pour } h \geqslant 3 m)
$$

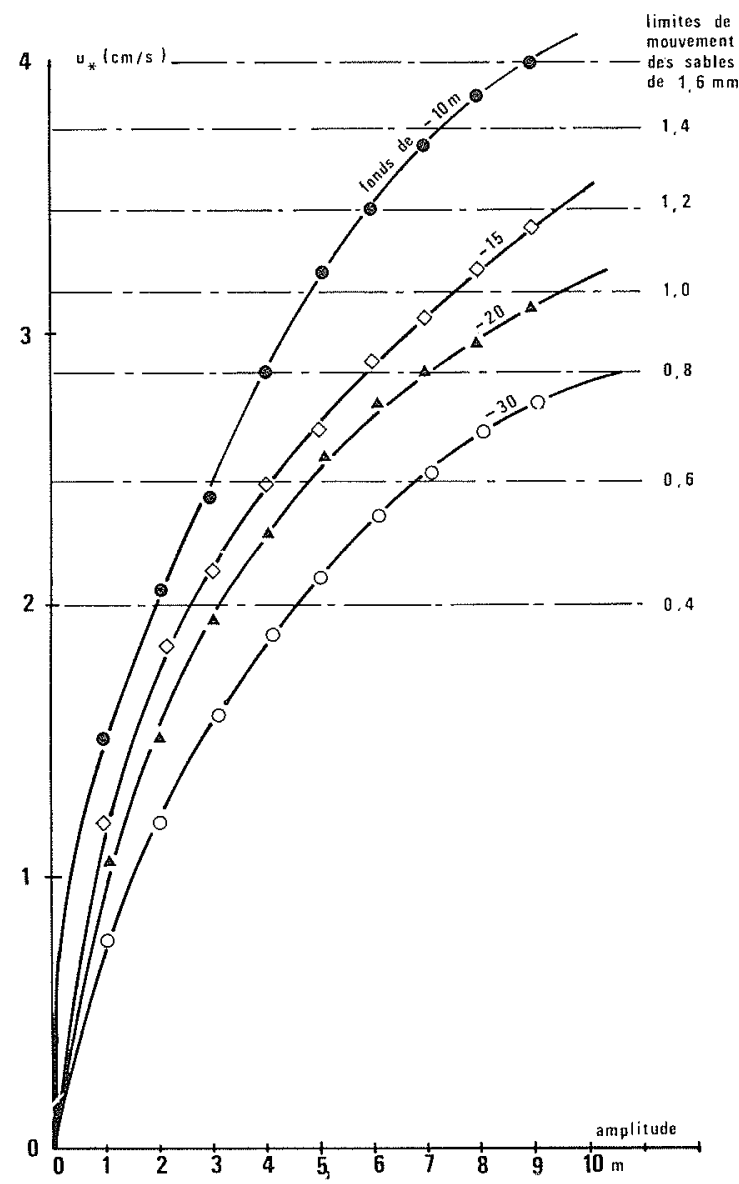

Figure 31 - Variation de la vitesse de frottement $u_{*}$ en fonction de l'amplitude de la houle

On constate que :

- à des profondeurs de $10 \mathrm{~m}$ la vitesse de frottement varie de $2 \mathrm{~cm} / \mathrm{s}$ pour $h=2 \mathrm{~m}$ et $T=9 \mathrm{~s}$, à $4 \mathrm{~cm} / \mathrm{s}$ pour $h=9 \mathrm{~m}$ et $T=17 \mathrm{~s}$; pour une houle de $5 \mathrm{~m}$ elle atteindrait $3,25 \mathrm{~cm} / \mathrm{s}$,

- à des profondeurs de $30 \mathrm{~m}$ la vitesse de frottement n'est plus que de $1,2 \mathrm{~cm} / \mathrm{s}$ pour $h=2 \mathrm{~m}$ et 2,75 $\mathrm{cm} / \mathrm{s}$ pour $h=9 \mathrm{~m}$.

\section{Courants de translation et de compensation}

Oscillant alternativement sur les fonds, sous l'action de la houle, les molécules d'eau peuvent être soumises en plus à des déplacements du large vers la côte dans les courants de translation qui prennent naissance dans la couche limite lorsque cette dernière est laminaire (figure 32). Dans ce cas, à chaque mouvement d'oscillation, les molécules pourraient avoir une composante de déplacement vers la côte, proportionnelle à la vitesse moyenne $U_{c l}$ déterminée d'après $P$. Lhermitte par l'expression :

$$
U_{c l}=K U_{\max }^{1,6} H^{-0.6}
$$

avec $k=1: 18$ en unités C.G.S. 


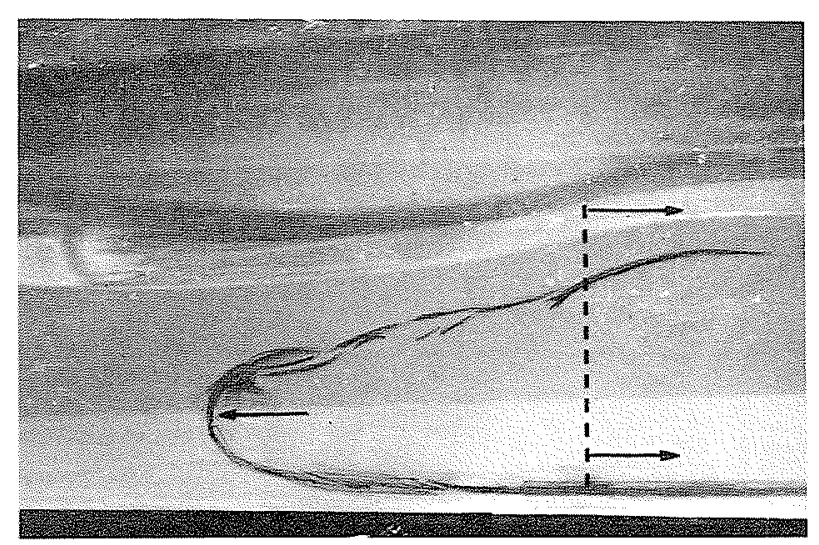

ligure 32 - Courant d'entrainement dans la masse du fluide (couche limite laminaire)

Par fonds de $H=20 \mathrm{~m}$, une houle de $4 \mathrm{~m}$ d'amplitude et $10 \mathrm{~s}$ de période engendrerait un courant de translation de $1,27 \mathrm{~cm} / \mathrm{s}$ susceptible de transporter des fines particules sur une distance de $45 \mathrm{~m}$ en 1 heure. Précisons que ce courant correspond au maximum de la vitesse près de la paroi et que $P$. Lhermitte a démontré que le courant de masse près du fond n'était égal qu'à :

$$
u_{e}=1 / 1,6 u_{c l}
$$

Si la couche limite devient turbulente (figure 33), ce qui est le cas le plus souvent en tempête ou lorsque l'action du vent se superpose à la houle, il n'existe plus de courants de translation au voisinage du fond mais des bouffées de turbulence qui peuvent contribuer à mettre les sédiments en suspension sur une certaine épaisseur au-dessus des fonds. Les particules pourront alors être entraînées vers le large par les courants de compensation.

La limite théorique entre une couche limite laminaire et une couche limite turbulente est assez difficile à préciser. Les équations actuelles, déduites la plupart du temps d'essais en modèle réduit, ne donnent pas des résultats applicables à la nature où l'on constate que, même en tempête, des particules de sable peuvent être remontées vers la côte dans le courant de translation. On a trouvé, au large du littoral des Landes, que la vitesse de déplacement des sables vers la côte pouvait atteindre $30 \mathrm{~m}$ par jour en moyenne par des fonds de $-8 \mathrm{~m}$ et 5 à $6 \mathrm{~m}$ par des fonds de $-15 \mathrm{~m}$, les mesures étant faites sur une période de deux mois avec des houles pouvant dépasser la force 4 pendant $25 \%$ du temps. Pour des silts par contre, qui peuvent se détacher de la couche limite sous l'effet des mouvements orbitraires, les déplacements s'effectuent préférentiellement vers le large.

\section{Déferlement de la houle}

En arrivant dans la zone des petits fonds la houle déferle dès que la profondeur $\mathrm{H}$ est théoriquement égale à 1,28 fois l'amplitude de la houle au point considéré. En fait cette profondeur critique de déferlement dépendra non seulement de l'amplitude de la houle mais de sa période et de la pente des fonds. Pour une

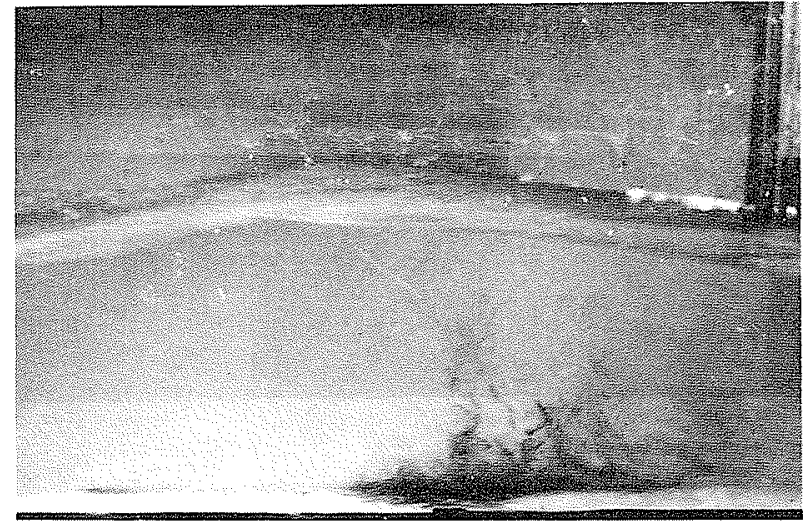

Figure 33 - Couche limite turbulente

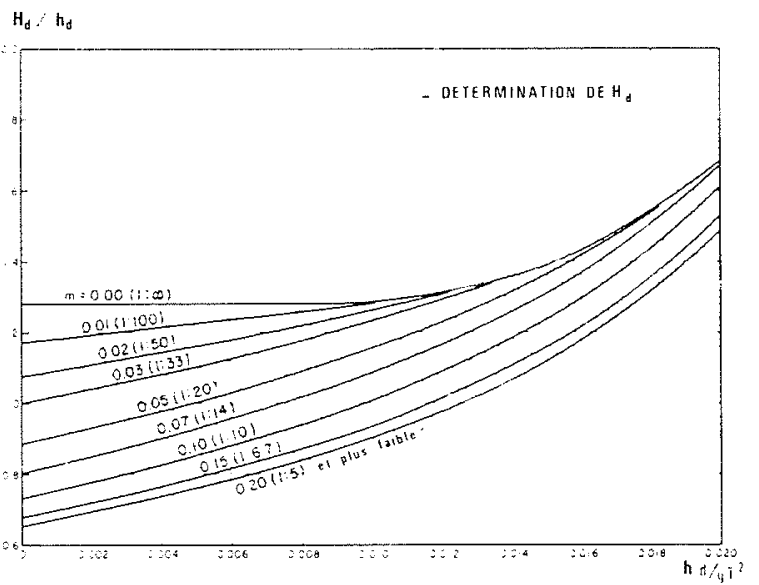

Figure 34 - Détermination de la profondeur de déferlement de la houle

pente des fonds inférieure à $1: 20$ la houle pourra déferler lorsque la profondeur sera égale à l'amplitude (figure 34).

Les vitesses instantanées dans la lame déferlante sont très importantes et entraînent des projections d'eau vers la côte contrebalancées par des courants de retour au voisinage du fond qui peuvent ramener les sédiments côtiers vers ce véritable pôle d'attraction sédimentaire que représente le déferlement (Figures 35 et 36).

Par ailleurs on constate entre le déferlement et la côte une surélévation épisodique du niveau moyen de l'eau par suite de la fluctuation des amplitudes dans les vagues qui se propagent sous forme de trains d'ondes. On peut estimer que la fluctuation du niveau de l'eau atteint $1 / 10$ à $1 / 15^{\mathrm{e}}$ environ de la différence entre les amplitudes des vagues successives. Si les vagues d'un train d'ondes varient entre 2 et $5 \mathrm{~m}$ la fluctuation du niveau moyen pourra atteindre 0,20 à $0,30 \mathrm{~m}$ entraînant non seulement des courants de retour importants mais pouvant être à l'origine des seiches qui se font ressentir dans les ports et les embouchures.

\section{Courant littoral ou courant de houle}

Sous l'action des houles obliques un courant de houle prend naissance et se dirige parallèlement à la côte for- 


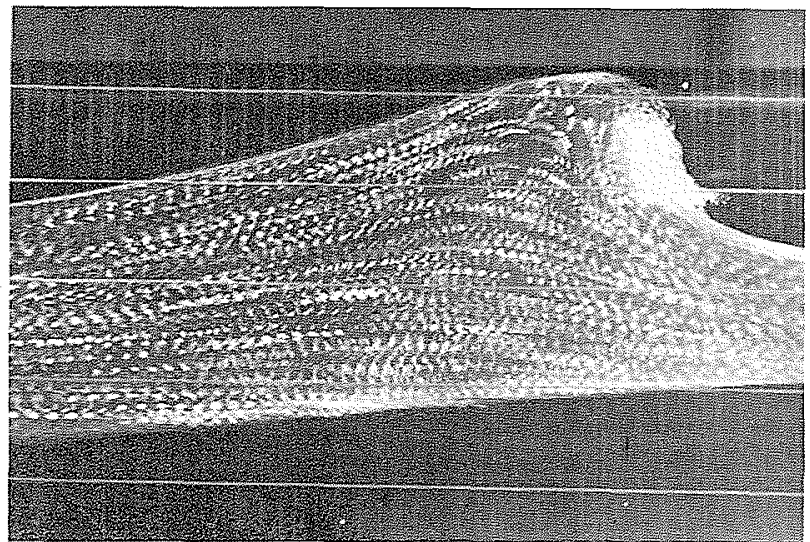

Figure 35 - Déferlement de la houle

mant un véritable "fleuve littoral" susceptible de transporter en suspension, ou par charriage, des masses considérables de sédiments apportés du large par le courant de translation sur les fonds ou arrachés à la côte par la houle.

La répartition des vitesses dans ce fleuve littoral, créé par les courants de houle, montre un maximum vers la côte entre une demi longueur d'onde et une longueur d'onde du point de déferlement. Vers le large les vitesses diminuent très rapidement pour devenir faibles à négligeables au-delà du point de déferlement de la houle. Vers la côte la diminution est plus lente comme le montre la figure 37 extraite de mesures faites en 1950 au L.C.H.F. dans une cuve à houle .

De nombreuses formules ont été proposées pour déterminer la vitesse des courants de houle en fonction des différents paramètres caractérisant la houle et les fonds sous-marins.

Le C.E.R.C. en se basant sur la formule de LonguetHiggins propose :

$$
V=20,7 i(g h)^{1 / 2} \sin 2 \alpha
$$

Par ailleurs J. Larras en utilisant les mesures faites par Putman estime que l'on peut avoir une approximation acceptable en prenant:

$$
V=2,6\left(\frac{g h^{2} i \sin 2 \alpha}{T}\right)^{1 / 3}
$$

avec :

$h=$ amplitude de la houle déferlante,

$i=$ pente moyenne de la plage (exprimée parsa tangente),

$\alpha=$ obliquité des lames du large avec le rivage.

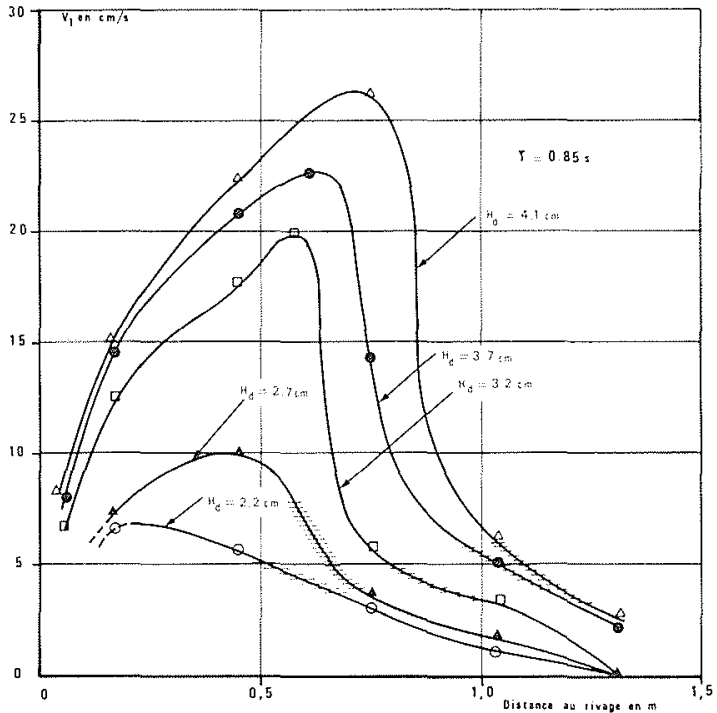

Figure 37 - Répartition des vitesses du courant de houle à différentes distances de la côte.

Pour une houle de tempête de $7 \mathrm{~m}$ d'amplitude et $12 \mathrm{~s}$ de période, la vitesse du courant de houle atteindrait $1,70 \mathrm{~m} / \mathrm{s}$ pour une obliquité des lames de $10^{\circ}$ avec le rivage ; cette valeur est compatible avec les observations en nature. Pour ces caractéristiques le débit liquide engendré par les courants de houle parallèlement à la cote correspondrait à celui d'un grand fleuve en crue avec 2000 à $3000 \mathrm{~m}^{3} / \mathrm{s}$

\section{Courants de retour ou "d'undertow" et "rip-currents"}

Les fluctuations du niveau de l'eau entre le déferlement et la côte peuvent, comme il a été dit, engendrer des accumulations et des vidages d'eau avec une période qui sera égale à celles des trains d'ondes de la houle, c'est-à-dire de 2 minutes en moyenne.

Les masses d'eau en mouvement par ce processus sont importantes et les échanges dans la zone de déferlement peuvent atteindre 40 à $50 \mathrm{~m}^{3}$ par mètre linéaire de plage, au cours des tempêtes, qui s'évacueront vers le large en une trentaine de secondes. Ces "courants de retour", proches du fond, peuvent être accentués lorsque le vent souffle du large et sont fréquemment appelés "undertow".

Ces courants de retour peuvent se concentrer en des points privilégiés, séparés de façon régulière, et formeront les "rip-currents" qui prendront naissance aux

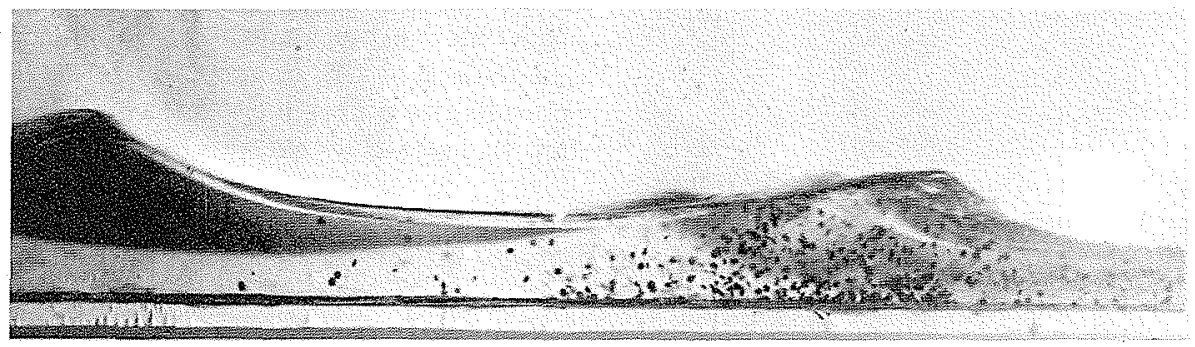

Figure 36 - Accumulation des matériaux dans le déferlement 


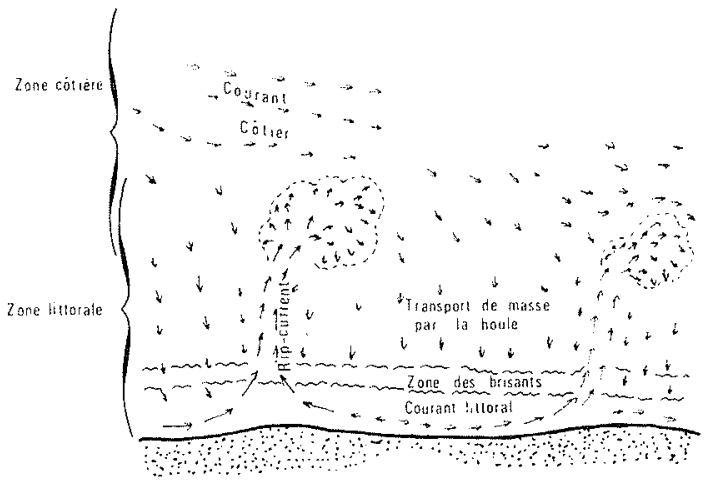

Figure 38 - Schéma général des courants près de la côte

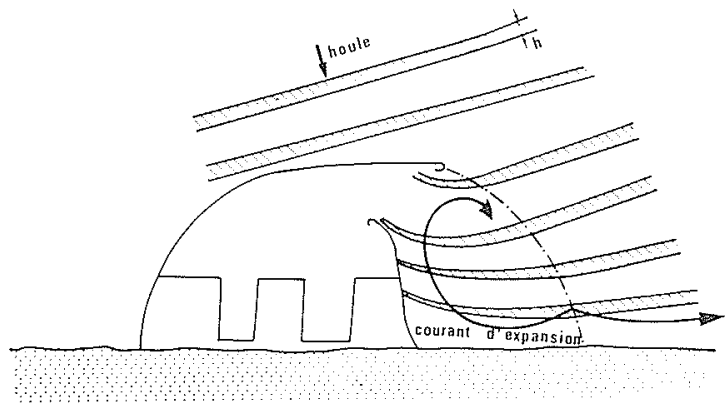

Figure 39 - Formation d'un courant d'expansion à l'abri d'un ouvragc

points où l'amplitude de la houle est la plus faible (figure 38)

Sur une plage rectiligne l'espacement des rip-currents varierait comme le carré de la période et serait sensiblement égal à la longueur d'onde des "edge-waves".

Les vitesses dans les rip-currents peuvent être très élevées et atteindre plusieurs mètres par seconde, l'action de "jet" se faisant sentir au-delà du déferlement et pouvant être à l'origine de certaines formations sédimentaires.

\section{Courants d'expansion latérale}

Ce type de courant est dû au déplacement des masses d'eau d'une zone où l'amplitude de la houle est forte vers une zone où l'amplitude est plus faible.

Un tel phénomène se produit lorsque l'on est en présence d'une topographie des fonds qui conduit à des concentrations de la houle en certains points ou à l'ombre d'ouvrages portuaires qui apportent, par effet de diffraction, des modifications dans la propagation de la houle (figure 39).

Les vitesses dans ces courants d'expansion latérale peuvent atteindre 0,5 à $1 \mathrm{~m} / \mathrm{s}$ et sont à l'origine de certains colmatages des passes d'entrée portuaires.

\section{Mouvements sédimentaires engendrés par la houle perpendiculairement au littoral}

Les mouvements engendrés par la houle dans la masse fluide, que ce soit les mouvements orbitaires sur les fonds, les courants de translation et de compensation, les courants littoraux ou de retour..., vont réagir sur les sédiments provoquant dans certains cas leur déplacement en masse vers des secteurs privilégiés.

Ces mouvements sédimentaires s'effectueront de façon très différente suivant les caractéristiques des matériaux, la profondeur à laquelle ils se trouvent et les conditions océanographiques existantes.

C'est leur comportement sous l'action des forces hydrodynamiques que nous aborderons dans ce paragraphe en signalant toutefois que, là encore, la complexité des phénomènes est telle que l'établissement des lois n'a pu se faire sans le concours de mesures en laboratoire et d'observations en nature.

Mise en oscillation des sédiments sous l'action des mouvements orbitaires

\section{Matériaux non cohésifs}

Les matériaux non cohésifs du type sables, graviers et galets pourront être mis en mouvement dès que les vitesses des courants alternatifs développés par la houle sur les fonds seront supérieures à la vitesse critique d'entraînement des sédiments.

En fait dans la plupart des matériaux les mouvements orbitaires ne seront pas limités à l'interface sol-eau mais pourront se propager à l'intérieur de la masse sédimentaire provoquant des sous-pressions favorables à la mise en mouvement des particules.

De nombreux auteurs ont essayé de rattacher le dé. but d'entrainement des particules de différents diamètres " $d$ " à la vitesse maximale $U_{\max }$ engendrée par la houle sur les fonds. On peut comparer quelques unes de ces formules en les mettant sous une même forme qui fait intervenir la densité des matériaux sous l'eau " $a$ ", la viscosité " $y$ " le diamètre des particules " $d$ " ou la vitesse de chute " $W$ " et la période de la houle " $T$ ".

- $U_{o}$ crit $=32,4 a^{2 / 3} d^{0,433} T^{1 / 3}$

en unités $\mathrm{cgs}$ (Bagnold - laminaire).

- $U_{o}$ crit $=0,025(a g) \nu^{-1 / 2} T^{1 / 2} d$

(Manohar - laminaire)

- $U_{o}$ crit $=7,45(a g)^{0,4} \nu^{0,2} d^{0,2}$

(Manohar - turbulent)

$-U_{0}$ crit $=W+95 a^{1 / 3} \nu^{1 / 2} T^{-1 / 2}$

(Larras - laminaire)

$-U_{0}$ crit $=0,33(a g)^{2 / 3} \nu^{1 / 24} T^{3 / 8} d^{1 / 4}$

(Goddet - laminaire)

- $U_{o}$ crit $=1,73(\rho-\rho o)^{1 / 2} g^{1 / 2} d^{1 / 2}$

en unités c g s (Goddet - turbulent)

$-U_{o}$ crit $=0,175(a g)^{3 / 4} T^{1 / 2} d^{1 / 4}$

(Valembois - laminaire)

$-U_{o}$ crit $=0,11(a g)^{4 / 5} \nu^{-1 / 10} T^{1 / 2} d^{2 / 5}$

(Moguilny - laminaire)

- $U_{o}$ crit $=0,385(a g)^{2 / 3} T^{1 / 3} d^{1 / 3}$

(Sato - laminaire) 


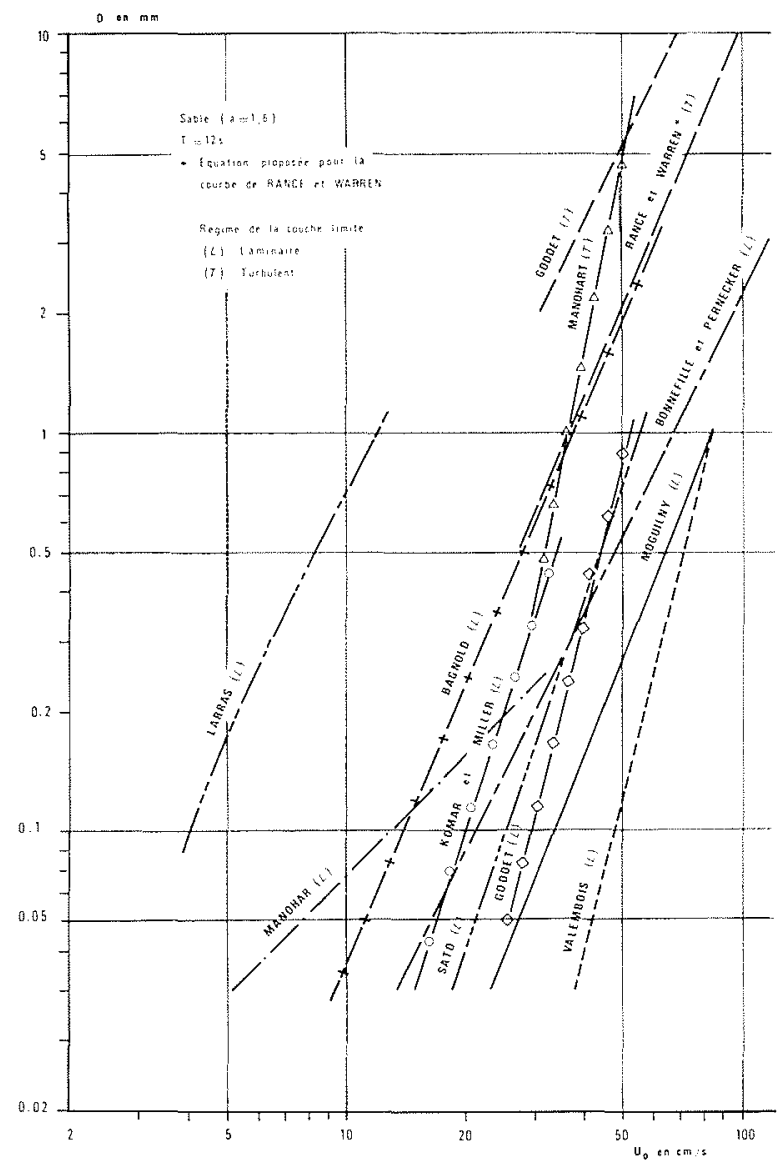

Figure 40 - Début d'entraînement des sédiments non cohésifs sous l'action de la houle. Comparaison des différentes équations

$$
\begin{gathered}
-U_{o} \text { crit }=0,063(\mathrm{ag})^{5 / 6} \nu^{-1 / 6} T^{1 / 2} d^{1 / 2} \\
\text { (Bonnefille et Pernecker - laminaire) } \\
-U_{O} \text { crit }=1,05(\mathrm{ag})^{4 / 7} T^{1 / 7} d^{3 / 7} \\
\text { (Rance et Warren - turbulent) } \\
-U_{o} \text { crit }=0,30(\mathrm{ag})^{2 / 3} T^{1 / 3} d^{1 / 3} \\
\text { (Komar et Miller - laminaire) }
\end{gathered}
$$

La figure 40 donne un aperçu des écarts que l'on peut obtenir entre les différentes formules basées sur la vitesse horizontale maximale sur le fond.

Au L.C.H.F. on a admis, depuis 1950, que pour qu'il y ait début d'oscillation de particules sableuses sur les fonds il faut que la force tractrice moyenne $\tau_{0}$ développée par la houle soit supérieure à la force tractrice critique $\tau$. d'entrainement des matériaux sous l'action des courants.

Sachant que :

$$
\tau_{c}=50\left(\rho_{s}-\rho_{0}\right) d g, \text { avec } \tau_{c} \text { en } N / \mathrm{m}^{2} \text { et } d \text { en mètres }
$$
et que :

$$
\tau_{0}=u_{*}^{2} \rho=1.000\left(\frac{8 \nu U_{\text {max }}^{2}}{\pi T}\right)^{1 / 2}
$$

on en déduit, dans le cas de sable, et d'une eau ayant une viscosité de $10^{-6} \mathrm{~m}^{2} / \mathrm{s}$, que :

$$
\frac{U_{\max }}{T^{1 / 2}} \geqslant 500 d
$$

On peut également utiliser, comme pour les courants, des paramètres addimensionnels en :

$$
\begin{aligned}
& d_{*}=\left(\epsilon g / \nu^{2}\right)^{1 / 3} d \\
& \operatorname{Re}=u_{*} d / \nu
\end{aligned}
$$

et utiliser le même abaque que celui de début d'entraînement des sédiments sous l'action d'un courant uniforme dont les limites sont, rappelons-le :

$$
d_{*}=2,5 R e_{*}^{4 / 5} \quad \text { et } \quad d_{*}=3,8 R e_{*}^{5 / 8}
$$

A titre d'exemple, par fonds de $12 \mathrm{~m}$, la limite des mouvements des particules de diamètre $d$ donné, pour différentes amplitudes, est indiquée ci-après:

\begin{tabular}{|c|c|c|c|c|c|c|c|}
\hline $\begin{array}{c}h \\
\text { (amplitude en } \\
\mathrm{m})\end{array}$ & 1 & 2 & 3 & 4 & 5 & 6 & 7 \\
\hline$d(\mathrm{~mm})$ & 0,06 & 0,1 & 0,4 & 0,57 & 0,73 & 0,8 & 1,2 \\
\hline
\end{tabular}

Pour les houles de la côte du Golfe de Gascogne on constate en appliquant ces formules que (figure 31) :

- par les profondeurs de - $10 \mathrm{~m}$ sous le niveau de l'eau les particules supérieurs à $2,0 \mathrm{~mm}$ ne pourront être déplacées quelle que soit l'amplitude des houles. Par contre, à partir d'une amplitude de $5 \mathrm{~m}$, les particules de $1 \mathrm{~mm}$ seront mises en mouvement et il suffira d'une houle de $2 \mathrm{~m}$ pour provoquer le début d'oscillation des particules fines comprises entre 0,4 et $0,2 \mathrm{~mm}$;

- par des profondeurs de - $20 \mathrm{~m}$ les particules supérieures à $1 \mathrm{~mm}$ ne se déplaceront pas quelles que soient les houles et il faudra que l'amplitude dépasse $5 \mathrm{~m}$ pour déplacer des grains de $0,6 \mathrm{~mm}$. Par contre les sables fins de 0,4 à $0,2 \mathrm{~mm}$ subiront un début d'oscillation à partir d'une amplitude de 3 à $3,50 \mathrm{~m}$ (10\% du temps);

- par des profondeurs de - $30 \mathrm{~m}$ il faudra que les houles dépassent $4,50 \mathrm{~m}$ pour provoquer le début d'oscillation des sables fins ( $3 \%$ du temps), les sables plus grossiers ou ayant une cohésion propre (sables de $0,10 \mathrm{~mm}$ et inférieurs) restant pratiquement stables.

\section{Matériaux cohésifs (vases et limons)}

Sur des fonds de vase la houle développe des mouvements orbitaires d'autant plus importants que la rigidité de la vase est plus faible. On retrouve comme pour les courants l'influence du facteur rigidité $\tau y$ sur le début d'entrainement des particules de vase ainsi que du facteur viscosité.

Dans une vase dont la viscosité est inférieure à 100 cps il peut se développer, sous l'action de la houle, des mouvements oscillatoires périodiques qui ne correspondent plus aux équations classiques $d u$ fluide parfait car il est indispensable de faire intervenir les termes de viscosité dans les équations de Navier-Stokes.

D'après les expériences faites au L.C.H.F. on constate que l'influence du terme de viscosité se traduit près de l'interface par une décroissance très rapide de l'ampli- 
tude du mouvement, alors qu'au contraire, dans un fluide non visqueux l'amortissement est extrêmement progressif. Dans les deux cas on retrouve un mouvement elliptique des particules qui se propage à une profondeur d'autant plus faible que la viscosité de la vase est grande et l'amplitude de la houle plus petite.

La figure 41 illustre ces résultats et montre que : - l'amplitude des mouvements orbitaires décroit bruta- lement à l'interface entre le fluide sain et la vase puis progressivement ensuite dans la masse de la vase ;

- la décroissance des mouvements orbitaires est d'autant plus rapide que la viscosité de la vase est élevée;

- pour une même vase l'amplitude des mouvements à une profondeur donnée est d'autant plus importante que l'amplitude de la houle est grande.
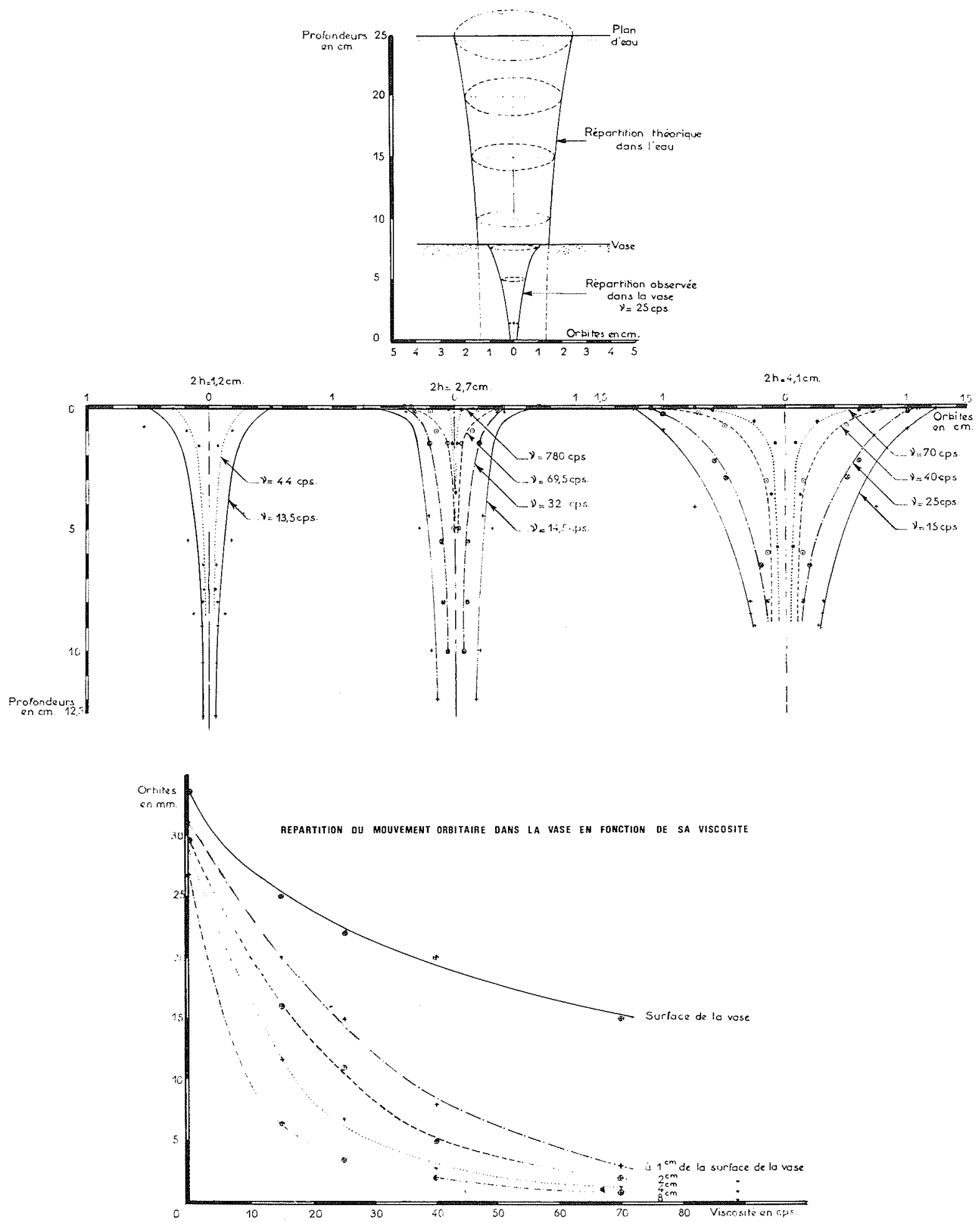

Figure 41 - Mouvement des vases sous la houle 
Importance des volumes de matériaux brassés par la houle à différentes profondeurs - Estimation des quantités de sable mis en suspension

Importance des volumes de matériaux brassés par la houle.

On peut présumer, par la théorie, l'importance des mouvements sédimentaires à différentes profondeurs en effectuant à partir de la vitesse de frottement $u_{*}$ et des équations de transport sous l'action des courants (Meyer-Peter, Einstein . . .) une estimation des quantités de matériaux brassés sur place par la houle. Par ailleurs connaissant le pourcentage des différentes houles qui règnent au cours de l'année il est possible de faire un bilan annuel des possibilités de mise en mouvement à différentes profondeurs d'un littoral et d'évaluer les risques de colmatage d'une souille de dragage par exemple.

Les résultats obtenus pour le littoral du Golfe de Gascogne illustrent cette méthode d'évaluation et permettent de se faire une idée de la diminution des mouvements sédimentaires, en fonction de la profondeur (figure 42).

\begin{tabular}{|c|r|r|r|r|r|}
\hline \multicolumn{6}{|c|}{$\begin{array}{c}\text { Comparaison des volumes théoriques remaniés } \\
\text { par la houle d'après des formules type Meyer Peter } \\
\text { et Einstein }\end{array}$} \\
\hline $\begin{array}{c}\text { Profondeur } \\
\text { (en m) }\end{array}$ & 7 & 10 & 15 & 20 & 30 \\
\hline $\begin{array}{c}\text { Volumes remaniés } \\
\text { en m } 3 / \text { m/an } \\
\text { (Meyer Peter) }\end{array}$ & 385 & 38 & 13 & 5,5 & 0,5 \\
\hline (Einstein) & 180 & 72 & 19 & 10 & 2 \\
\hline
\end{tabular}

Sans vouloir attacher une valeur quantitative et absolue aux résultats de ces calculs, on constate que :

- les quantités de sable fin susceptibles d'être remaniés par l'action des houles diminuent très rapidement avec la profondeur; elles sont encore importantes par fonds de $-10 \mathrm{~m}$ et pratiquement nulles par fonds de $-30 \mathrm{~m}$;

- on peut estimer que par fonds de $-30 \mathrm{~m}$ les risques de remaniement des fonds sont en moyenne 50 fois plus faibles que par fonds de $-10 \mathrm{~m}$;

- par fonds de $-15 \mathrm{~m}$, par contre, les remaniements restent appréciables ;

- plus on se rapproche du littoral plus les houles de faible amplitude interviennent dans le remaniement global des fonds (figure 43).

\section{Estimation des quantités de sable remis en s̈uspension}

Pour certaines conditions de houle on assiste à une mise en suspension des sédiments constituant le fond de la mer.

La quantité de matériau remis en suspension est importante puisque, dans certains cas, elle pourra condamner des prélèvements d'eau pour le refroidissement d'installations industrielles telles que les centrales électriques.

Le phénomène de remise en suspension est bien dif. férent suivant que l'on se place dans la zone de défer. lement ou au large de celle-ci. Dans les brisants, le champ des vitesses est tel qu'il se produit une turbulence généralisée sur toute la hauteur d'eau, ce qui conduit à des concentrations des matériaux en suspension souvent très élevées (plusieurs $\mathrm{g} / \mathrm{l}$ ). Au con-

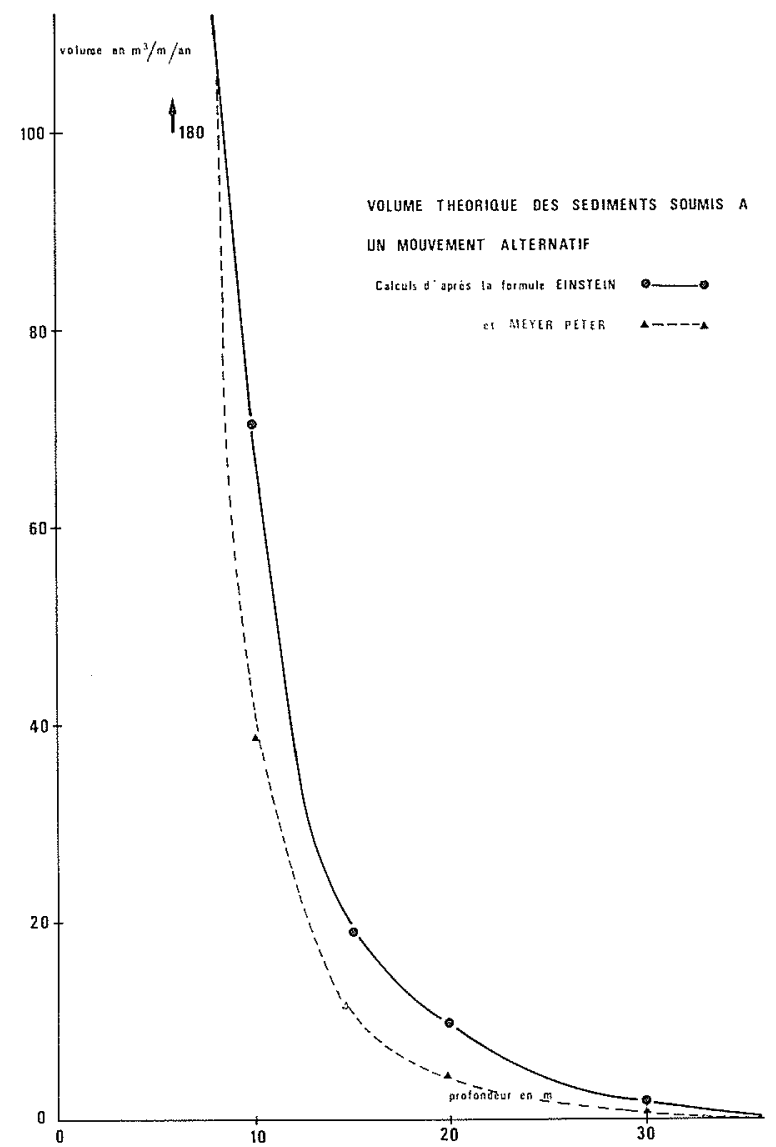

Figure 42 - Etude théorique de l'action des houles sur les fonds du Golfe de Gascogne

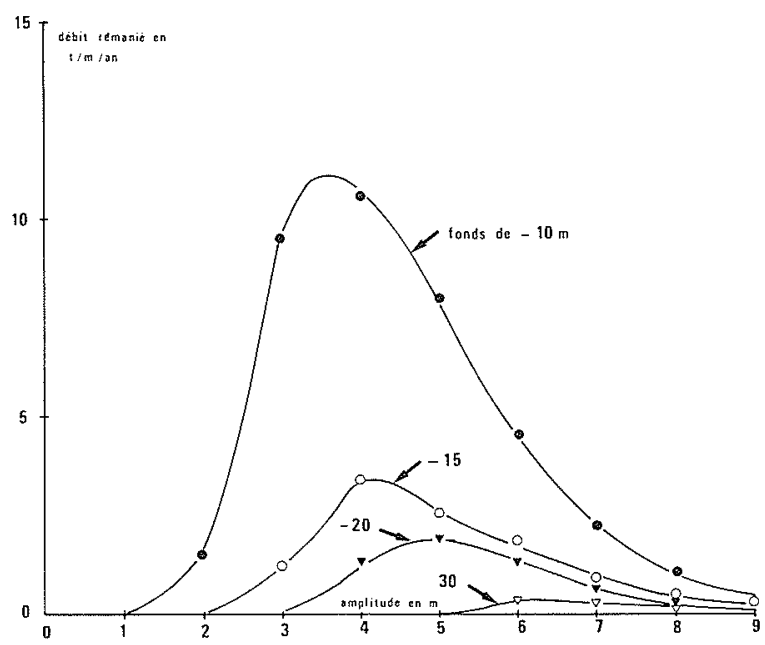

Figure 43 - Graphique des remaniements théoriques à différentes profondeurs en fonction de l'amplitude de la houle 
traire, au large du déferlement, la seule source de turbulence susceptible de mettre les matériaux en suspension est la couche limite et on ne rencontre de fortes concentrations que près du fond.

La théorie ne rend pas compte d'une façon correcte de la répartition des concentrations au-dessus des fonds et, dans l'état actuel, on doit se rattacher à des valeurs mesurées en nature pour essayer d'avoir une idée de ce problème.

Le Beach Erosion Board signale que dans le déferlement, des mesures ont donné des concentrations de $17 \mathrm{~g} / 1$ et qu'à $8 \mathrm{~m}$ seulement du déferlement la teneur n'était plus que de $4 \mathrm{~g} / 1$ pour tomber à $1 \mathrm{~g} / 1$ à $80 \mathrm{~m}$. Pour la zone étudiée les concentrations de sable n'avaient pas dépassé $2 \mathrm{~g} / 1$ à plus de $0,60 \mathrm{~m}$ audessus du fond.

On retrouve un peu les mêmes résultats dans les études de Fukushima faites à l'aide de pièges fixés à différentes hauteurs, et d'une façon générale si l'on trouve des concentrations de $230 \mathrm{~g} / 1$ dans les brisants près du fond il apparaît que la teneur est faible au large à plus de $1,5 \mathrm{~m}$ du fond.

Toutes ces mesures sont malheureusement faites par des houles d'amplitudes assez faibles et il n'est pas possible dans l'état actuel de nos connaissances de définir les risques de trouver des sables fins en suspension à une certaine profondeur. Le fait de constater le long du littoral une frange très colorée de près de $1 \mathrm{krn}$ de largeur susceptible de contenir des sables en suspension au cours des fortes tempêtes conduit à une certaine prudence avant de négliger la remise en suspension des sables fins.

\section{Mouvements des particules solides perpendiculairement au littoral}

Mises en oscillations par les mouvements orbitaires de la houle, les particules solides pourront subir des déplacements dans le profil perpendiculairement au littoral, être remontées vers la côte dans les courants de translation ou arrachées des rivages par les lames déferlantes, une partie des éléments s'accumulant dans la barre au voisinage du déferlement tandis que les éléments très fins pourront être dispersés vers le large dans les courants de compensation.

\section{Matériaux non cohésifs}

\section{a) Observations en nature}

Les études effectuées par le L.C.H.F. dans différentes régions ont montré que les mouvements dans le profil étaient importants entre la côte et les fonds de -7 à - $10 \mathrm{~m}$. Dans cette zone, les fonds s'érodent et se sédimentent alternativement suivant les caractéristiques des houles, mais les profils moyens peuvent rester stables au cours des années (figure 44). Au total l'importance des mouvements alternatifs dans le profil atteint $500000 \mathrm{~m}^{3}$ par an et par kilomètre de plage (Méditerranée).

Un point singulier apparaît par fonds de $-7 \grave{a}-10 \mathrm{~m}$ où les fluctuations restent faibles bien que des échanges sédimentaires puissent s'effectuer entre le large et les petits fonds.

Entre les fonds de $-10 \mathrm{~m}$ et de $-15 \mathrm{~m}$ à $-20 \mathrm{~m}$ des variations plus faibles des profondeurs peuvent être observées, les matériaux étant soit emportés par les courants de compensation au cours des tempêtes, soit ramenés vers la côte au début de la période de beau temps. La résultante de ces deux mouvements transversaux se traduit, à chaque tempête, par une "perte" en matériaux fins qui sont entraînés vers les plus grandes profondeurs où ils ne peuvent plus être repris par les houles.

Il ne semble pas que des remaniements sédimentaires de grande importance puissent être observés au-delà des fonds de $-20 \mathrm{~m}$ et il faut rappeler que, dans la plupart des cas, les vases pures apparaissent à partir des fonds de -30 à $-40 \mathrm{~m}$ éliminant une possibilité d'alimentation en sable des zones côtières par les sédiments provenant des plus grandes profondeurs.

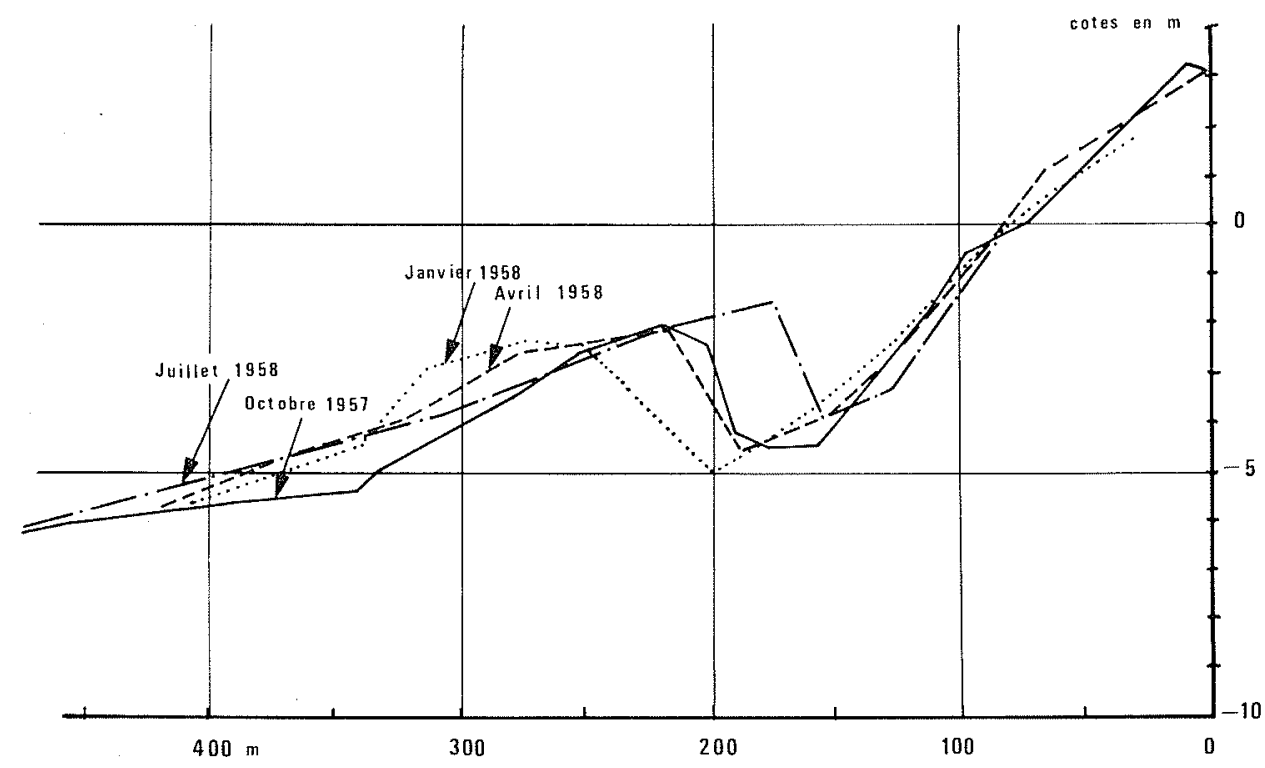

Figure 44 - Evolution saisonnière des profils 


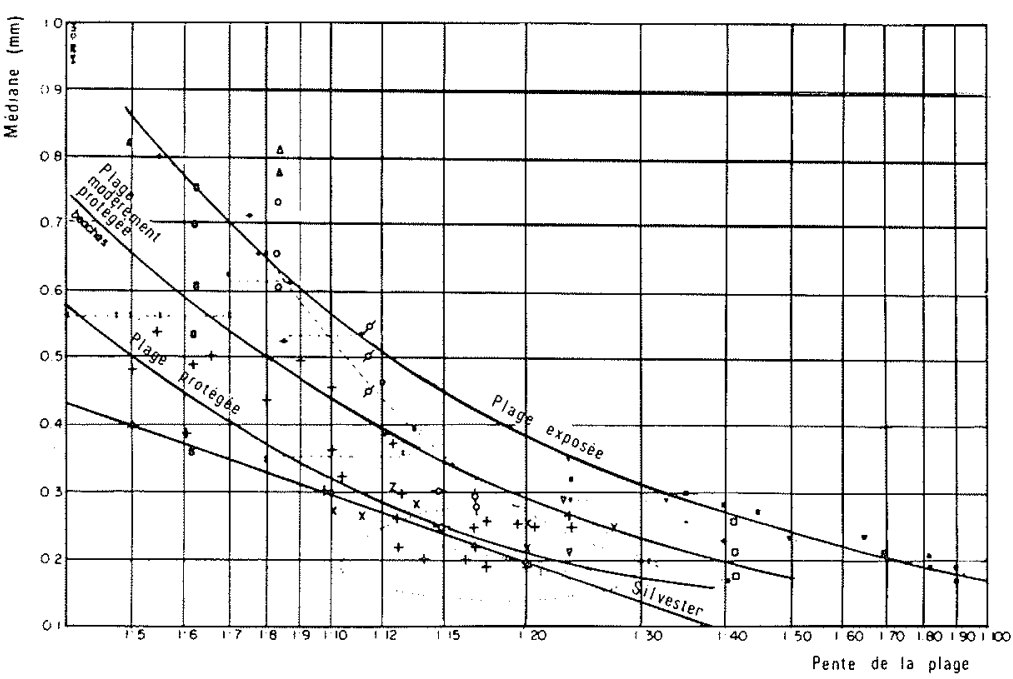

Figure 45 - Pente de la plage en fonction de la médiane et de l'exposition đ'après Wiegel

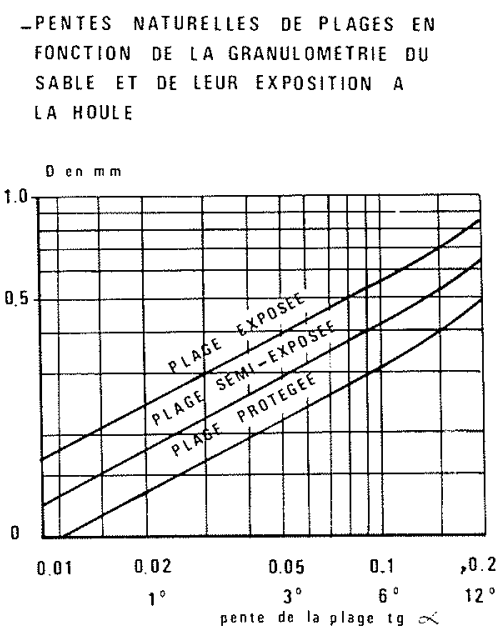

Figure 46 - d'après Sylvester

\section{b) Pentes des plages}

D'une manière générale la pente d'une plage dépendra de la granulométrie des sédiments et des caractéristiques de la houle. A diamètre égal la pente sera d'autant plus forte que la plage sera protégée des houles, à agitation égale la pente croît avec le diamètre.

Wiegel et Sylvester ont classé les pentes des plages en fonction de leur exposition et de leur granulométrie, les résultats figurant sur les figures 45 et 46 .

On peut également utiliser les valeurs du tableau ci-après qui montrent l'importance de la granulométrie d'un sédiment sur la pente moyenne des plages.

\begin{tabular}{|l|c|c|c|c|c|c|c|c|}
\hline \multicolumn{10}{|c|}{$\begin{array}{c}\text { Relation entre la pente d'une plage } \\
\text { et le diamètre des grains }\end{array}$} \\
\hline Diamètre en mm & 0,1 & 0,2 & 0,4 & 0,8 & 1,5 & 3 & 30 & 150 \\
\hline Pente en degrés & 1 & 3 & 5 & 7 & 9 & 11 & 17 & 24 \\
\hline
\end{tabular}

Dans le cas de rechargement de plage ou de réalisation de plages artificielles on aura intérêt à prendre un sédiment artificiel d'une granulométrie supérieure au sédiment en place afin d'obtenir une meilleure stabilité du profil et de réduire les volumes de matériaux à mettre en place.

Il faut également rappeler qu'il existe un triage granulométrique des sédiments dans le profil par suite des remontées des sables les plus grossiers vers le déferlement et du départ des éléments fins vers le large. Les nombreuses mesures faites au L.C.H.F. donnent les corrélations suivantes entre les profondeurs et les diamètres des grains :

\begin{tabular}{|c|c|c|c|c|c|}
\hline \multicolumn{6}{|c|}{ Triage granulométrique dans le profil } \\
\hline $\begin{array}{c}\text { Profondeur } \\
\text { en } m\end{array}$ & 0 & -5 & -10 & -15 & -30 \\
\hline $\begin{array}{c}\text { Diamètre } \\
\text { en } m m\end{array}$ & $\begin{array}{c}0,25 \\
\grave{a} \\
0,40\end{array}$ & $\begin{array}{c}0,15 \\
\text { à } \\
0,25\end{array}$ & $\begin{array}{c}0,10 \\
\text { à } \\
0,15\end{array}$ & $\begin{array}{c}0,05 \\
\text { à } \\
0,12\end{array}$ & $<0,05$ \\
\hline
\end{tabular}

c) Epaisseur de la couche mobile des sables et graviers

L'estimation des épaisseurs de sable déplacé par la houle est très importante à connaître et des mesures faites à l'aide de traceurs fluorescents ou radioactifs ont permis d'en fixer les ordres de grandeur.

D'après différents auteurs (Ingle, Kadib, King, L.C.H.F., SAPRA. . .) l'épaisseur " $E$ " de la couche mobile serait :

- sur une plage proportionnelle au diamètre des grains $d$ :

$$
E=37 d-0,58(\mathrm{si} E \text { et } d \text { exprimés en } \mathrm{cm})
$$

pour un sable de $1 \mathrm{~mm}$ l'épaisseur de la couche mobile atteindrait 3 à $4 \mathrm{~cm}$;

- entre les "brisants" et la côte, proportionnelle à l'amplitude de la houle déferlante "hd":

$E=0,03 h_{d}$ (E et $h_{d}$ en $\mathrm{cm}$ pour un sable de $0,25 \mathrm{~mm})$

cette valeur augmenterait avec le diamètre des grains, et semble plus vraisemblable ;

- sur un cordon de galets (Bas Champs) l'épaisseur de transport serait en moyenne de $60 \mathrm{~cm}$ (hd maximum $3 \mathrm{~m}$ ), par contre sur un estran de sable fin (La Coubre) l'épaisseur de la couche mobile n'atteindrait que $8 \mathrm{~cm}$. Avec de la madrague $(d=2,5$ à $4 \mathrm{~mm})$ l'épaisseur de la couche mobile sur l'estran pourrait atteindre $40 \mathrm{~cm}$;

- par des fonds de -6 et de - 9 m l'épaisseur de la couche mobile est de 10 et $3 \mathrm{~cm}$ pour des particules de 1,6 à $3 \mathrm{~mm}$. Avec des sables de $0,35 \mathrm{~mm}$ cette épaisseur serait négligeable par des fonds de -18 et $-15 \mathrm{~m}$ et atteindrait $10 \mathrm{~cm}$ par des fonds de $-10 \mathrm{~m}$;

- le L.C.H.F. et la SAPRA en effectuant des mesures continues pendant trois mois, en Méditerranée, avec des houles atteignant $3 \mathrm{~m}$ d'amplitude et pendant trois mois dans le Golfe de Gascogne avec des houles atteignant $7 \mathrm{~m}$, ont obtenu les résultats sui- 
vants qui montrent que l'épaisseur de la couche mobile diminue rapidement avec la profondeur et augmente avec la granulométrie.

\begin{tabular}{|c|c|c|c|c|c|c|}
\hline \multicolumn{6}{|c|}{ Epaisseur de la couche mobile de sable en Mediterranée } \\
\hline $\begin{array}{c}\text { Profondeurs } \\
\text { en } \mathrm{m}\end{array}$ & -18 & -15 & -8 & -6 & -5 & Estran \\
\hline $\begin{array}{c}\text { Diamètre } \\
\text { en mm }\end{array}$ & 0,15 & 0,15 & 0,45 & 0,15 & 0,15 & 0,15 \\
\hline $\begin{array}{c}\text { Epaisseur } \\
\text { E en cm }\end{array}$ & 0 & $\begin{array}{c}1 \grave{a} \\
2\end{array}$ & $\begin{array}{c}5 \mathrm{à} \\
10\end{array}$ & 15 & 25 & 12 \\
\hline
\end{tabular}

Epaisseur de la couche mobile de sable en Atlantique

\begin{tabular}{|c|c|c|c|c|c|c|}
\hline $\begin{array}{c}\text { Profondeurs } \\
\text { en } \mathrm{m}\end{array}$ & $-22,5$ & -15 & -15 & -8 & -6 & \\
\hline $\begin{array}{c}\text { Diamètre } \\
\text { en mm }\end{array}$ & 0,12 & 0,40 & 0,15 & 0,20 & 0,7 & \\
\hline $\begin{array}{c}\text { Epaisseur } \\
\text { E en cm }\end{array}$ & $\begin{array}{c}0,3 \text { à } \\
1,2\end{array}$ & 14 & $\begin{array}{c}0,8 \text { à } \\
2,0\end{array}$ & $\begin{array}{c}16 \text { à } \\
27\end{array}$ & $\begin{array}{c}30 \text { à } \\
50\end{array}$ & \\
\hline
\end{tabular}

Pour des sables l'épaisseur de la couche mobile serait de quelques centimètres à quelques dizaines de centimètres. Elle serait très faible pour les fonds supérieurs à $-15 \mathrm{~m}$ et maximale près du déferlement. Cette épaisseur apparaît d'autant plus importante que la houle est forte et que le diamètre des matériaux est plus important. Compte-tenu du nombre limité de mesures il faut toutefois rester prudent dans la généralisation de ces résultats.

\section{d) Importance des mouvements dans le profil}

Des expériences réalisées en nature à l'aide de traceurs radioactifs permettent d'apprécier l'importance des mouvements des sédiments non cohésifs perpendiculairement au littoral. On constate que :

-- des galets de 2 à $4 \mathrm{~cm}$ de diamètre ne bougent pas par des fonds de $-18 \mathrm{~m}$ en Manche (expériences de Crimore). Par fonds de $-12 \mathrm{~m}$, ils remonteraient vers la côte avec un débit de $500 \mathrm{~m}^{3}$ par an et par kilomètre de longueuret de $1500 \mathrm{~m}^{3} / \mathrm{an} / \mathrm{km}$ par fonds de $-9 \mathrm{~m}$. De tels débits représentent des mouvements extrêmement faibles (200 ans pour qu'un galet passe des fonds de $-12 \mathrm{~m}$ à des fonds de $-9 \mathrm{~m}$ );

- des particules de 2 à $3 \mathrm{~mm}$ de sable grossier ne sont pas remaniées par des houles de $6 \mathrm{~m}$ à $7 \mathrm{~m}$ d'amplitude lorsque ces particules sont déposées par des fonds de $-12 \mathrm{~m} \mathrm{SH}$ ( $14 \mathrm{~m}$ sous le niveau moyen). Les mouvements apparaissent par des fonds de $-9 \mathrm{~m}$ et l'entrainement est total par des fonds de $-5 \mathrm{~m}$ une partie des gros éléments étant remontée à la côte (expériences L.C.H.F. - SAPRA) ;

- des particules fines de 0,2 à $0,4 \mathrm{~mm}$ immergées par des fonds de $-7 \mathrm{~m}$ le long des côtes des Landes sont remontées à la côte par des houles de $2,5 \mathrm{~m}$ d'amplitude. Les quantités mises en mouvement restent cependant assez faibles (L.C.H.F.) ;

- des sables de 0,3 $\mathrm{mm}$ déposés par des fonds de - $14 \mathrm{~m}$ ne bougeraient pratiquement pas pour des houles de 3 à $4 \mathrm{~m}$ d'amplitude (Commissariat à l'Energie Atomique Brésilien 1973);

- des sables de 0,25 $\mathrm{mm}$ déposés au large du Cap Ferret ne bougeraient pratiquement pas pour des profondeurs supérieures à $-10 \mathrm{~m}$ bien que les houles aient agi pendant 6 mois avec des amplitudes atteignant $5 \mathrm{~m}$ (L.C.H.F.).

Les études les plus récentes effectuées en 1975 par la SAPRA donnent des valeurs quantitatives des mouvements sédimentaires entre les fonds de $-22 \mathrm{~m}$ et de $-6 \mathrm{~m}$ le long des côtes des Landes. Les déplacements des matériaux ont été suivis pendant trois mois d'automne. Au cours de cette période une tempête atteignant $7 \mathrm{~m}$ d'amplitude a été relevée ainsi que 4 petites tempêtes de 4,5 à $6 \mathrm{~m}$ d'amplitude ; le bilan d'énergie de la houle $\left(h^{2}\right.$ T.t) a atteint $420.10^{6} \mathrm{~m}^{2} \mathrm{~S}^{2}$ soit $20 \%$ environ du bilan annuel $\left(2,110^{9} \mathrm{~m}^{2} \mathrm{~S}^{2}\right)$ des houles du Golfe de Gascogne.

Il ressort de cette étude que :

- Des sables fins que l'on dépose artificiellement sur des fonds marins de 15 à $20 \mathrm{~m}$ peuvent être remaniés temporairement et transportés en tempête sur quelques centaines de mètres ( $1000 \mathrm{~m}$ au maximum dans le cas des expériences) tant qu'ils ne se sont pas incorporés aux sédiments naturels (influence de l'enchevêtrement des grains).

- Une fois incorporés aux sédiments naturels, et représentatifs de ces derniers, les traceurs montrent que la limite granulométrique de début de déplacement des grains se situe à $0,4 \mathrm{~mm}$ environ pour les profondeurs de $-15 \mathrm{~m}$ CM (c'est-à-dire -17 à $-18 \mathrm{~m}$ au

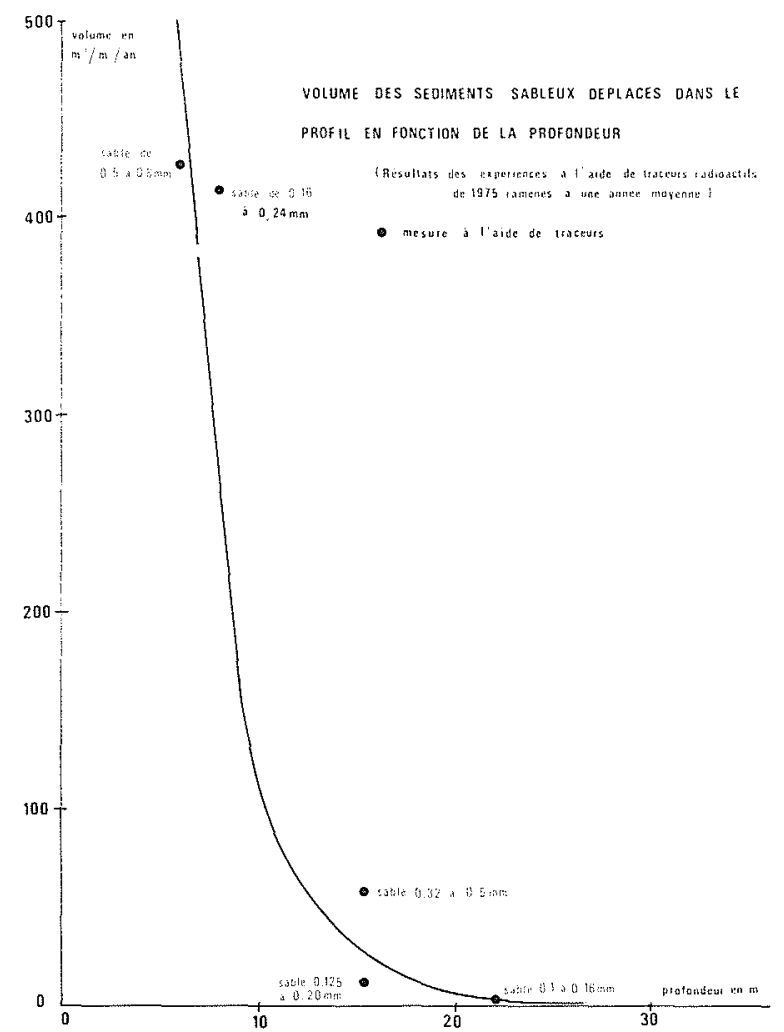

Figure 47 - Volume des sédiments sableux déplacés dans le profil en fonction de la profondeur 
niveau moyen) et à $0,8 \mathrm{~mm}$ pour les profondeurs de -6 à $-8 \mathrm{~m} \mathrm{CM}$.

Une partie des sédiments très fins peut être entrainée vers le large dans la couche limite turbulente entre les fonds de -6 à $-20 \mathrm{~m}$ dès que l'amplitude dépasse $7 \mathrm{~m}$; pour des amplitudes inférieures à $5,5 \mathrm{~m}$ le déplacement vers le large serait limité aux profondeurs de $-8 \mathrm{~m}$.

- Mis en oscillation par la houle, les sédiments plus grossiers sont remontés vers la côte mais les déplacements restent négligeables au-delà des fonds de - 12 à $-15 \mathrm{~m}$ (environ $1 \mathrm{~m}^{3} / \mathrm{m}$ en trois mois pour un bilan d'énergie de $420.10^{6} \mathrm{~m}^{2} \mathrm{~s}^{2}$.). Ces déplacements augmentent très rapidement lorsque l'on se rapproche de la côte (figure 47 .

- Les quantités transportées dans le profil, perpendiculairement à la côte, semblent proportionnelles au bilan d'énergie de la houle (Fig. 48). Pour un bilan de $42010^{6} \mathrm{~m}^{2} \mathrm{~s}^{2}$ ( 3 mois de houle) les quantités transportées aux différentes profondeurs sont données ciaprès :

\begin{tabular}{|c|c|c|c|c|c|}
\hline $\begin{array}{c}\text { Profondeur } \\
\text { (en m. CM) }\end{array}$ & -6 & -8 & $-15,2$ & $-15,5$ & $-22,2$ \\
\hline $\begin{array}{c}\text { Diamètre } \\
\text { des grains } \\
\text { (en mm) }\end{array}$ & $\begin{array}{c}0,5 \\
\grave{a}\end{array}$ & $\begin{array}{c}0,16 \\
\grave{a}\end{array}$ & $\begin{array}{c}0,125 \\
\text { à }\end{array}$ & $\begin{array}{c}0,3 \\
\grave{a}\end{array}$ & $\begin{array}{c}0,1 \\
\grave{a}\end{array}$ \\
\hline $\begin{array}{c}\text { Volume } \\
\text { transporté } \\
\text { (en } \mathrm{m}^{3} / \mathrm{m} \text { ) }\end{array}$ & 85 & 83 & 1,3 & 11,9 & 0,5 \\
\hline
\end{tabular}

Par fonds de $-8 \mathrm{~m}$ le volume transporté en $\mathrm{m}^{3} / \mathrm{m}$ serait donné par l'expression :

$$
V \mathrm{~m}^{3} / \mathrm{m}=f(p) \Sigma \mathrm{h}^{2} . T . t \text { avec } f(p)=2 \cdot 10^{-7} s^{-2}
$$

Par fonds de $-10 \mathrm{~m}$ la valeur de $f^{\prime}(p)$ ne serait plus que de $2.10^{-8} s^{-2}$ et tomberait à $0,7510^{-8} s^{-2}$ par fonds de $-15 \mathrm{~m}$.

Ces valeurs du coefficient $f(p)$ doivent être modulées pour tenir compte de la profondeur du déferlement. Pour des tempêtes de $7 \mathrm{~m}$ d'amplitude, susceptibles de déferler par des fonds de $9 \mathrm{~m}$ la valeur de $f(p)$ sera plus importante pour cette profondeur que pour des profondeurs plus faibles, où la houle a perdu une partie de son énergie.

- A une même profondeur les transports peuvent être plus importants pour des particules de 0,3 à $0,5 \mathrm{~mm}$ que pour des particules de 0,10 à $0,15 \mathrm{~mm}$ (réduction du transport par suite d'une certaine cohésion entre les grains).

- Si l'on extrapole l'ensemble des résultats obtenus sur trois mois à une année moyenne, on constate que les quantités transportées par fonds de $-8 \mathrm{~m}$ dans le profil, perpendiculairement à la côte, seraient de :

$$
V \mathrm{~m}^{3} / \mathrm{m} / \mathrm{an}=2 \cdot 10^{-7} \cdot 2,1 \cdot 10^{9}=420 \mathrm{~m}^{3} / \mathrm{m}
$$

valeur très voisine de celle que le L.C.H.F. avait indiquée à partir de l'étude de l'évolution des profils ( $500.000 \mathrm{~m}^{3}$ par kilomètre de plage).

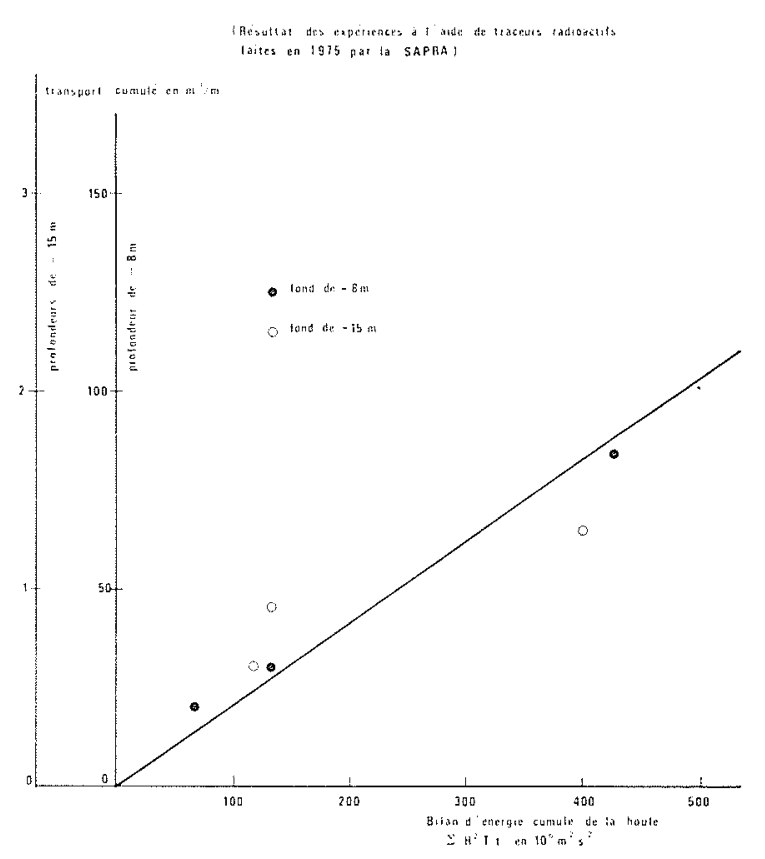

Figure 48 - Volume des sédiments sableux transportés dans le profil en fonction du bilan d'énergie de la houle

- Des courants généraux, parallèles au littoral, peuvent provoquer une dérive des sédiments mis en oscillation par les houles mais, dans le cas de la côte des Landes (vitesse des courants généraux de 0,15 à $0,20 \mathrm{~m} / \mathrm{s}$ ), les quantités transportées restent très faibles $\left(0,1\right.$ à $\left.0,8 \mathrm{~m}^{3} / \mathrm{m} / \mathrm{an}\right)$.

- Lorsque l'on se rapproche de la zone de déferlement, c'est-à-dire de la côte, on peut pour certaines houles observer un déplacement en masse et un étalement du traceur parallèlement au rivage, l'entraînement étant d'autant plus important que l'obliquité des houles est forte.

\section{Matériaux cohésifs}

\section{a) Résultats de mesures en laboratoire}

Sous l'action de la houle le développement d'un mouvement laminaire dans le fluide visqueux, que constitue la vase, se traduit par un mouvement de translation moyen des particules de vase dans le sens de la propagation de la houle présentant des analogies avec celui que l'on peut observer sur des fonds rigides.

La répartition des vitesses de translation moyenne dans le fluide sain et dans la vase est illustrée sur la figure 49 qui donne également la vitesse moyenne de déplacement de la vase en fonction de la profondeur, de la viscosité et de l'amplitude des houles étudiées.

On constate d'après ces résultats que :

- la vitesse de déplacement décroît très rapidement en fonction de la profondeur ;

- dans les conditions expérimentales $(d=25 \mathrm{~cm}$, $h$ de 0 à $5 \mathrm{~cm}$ ) la vitesse de déplacement $U_{v}$ de la vase en surface (viscosité de 30 à $70 \mathrm{cps}$ ) peut être liée à la vitesse théorique dans la couche limite sur un fond rigide $U_{c l}$ par une relation de la forme :

$$
\dot{U}_{v}=0,25 U_{c l}
$$



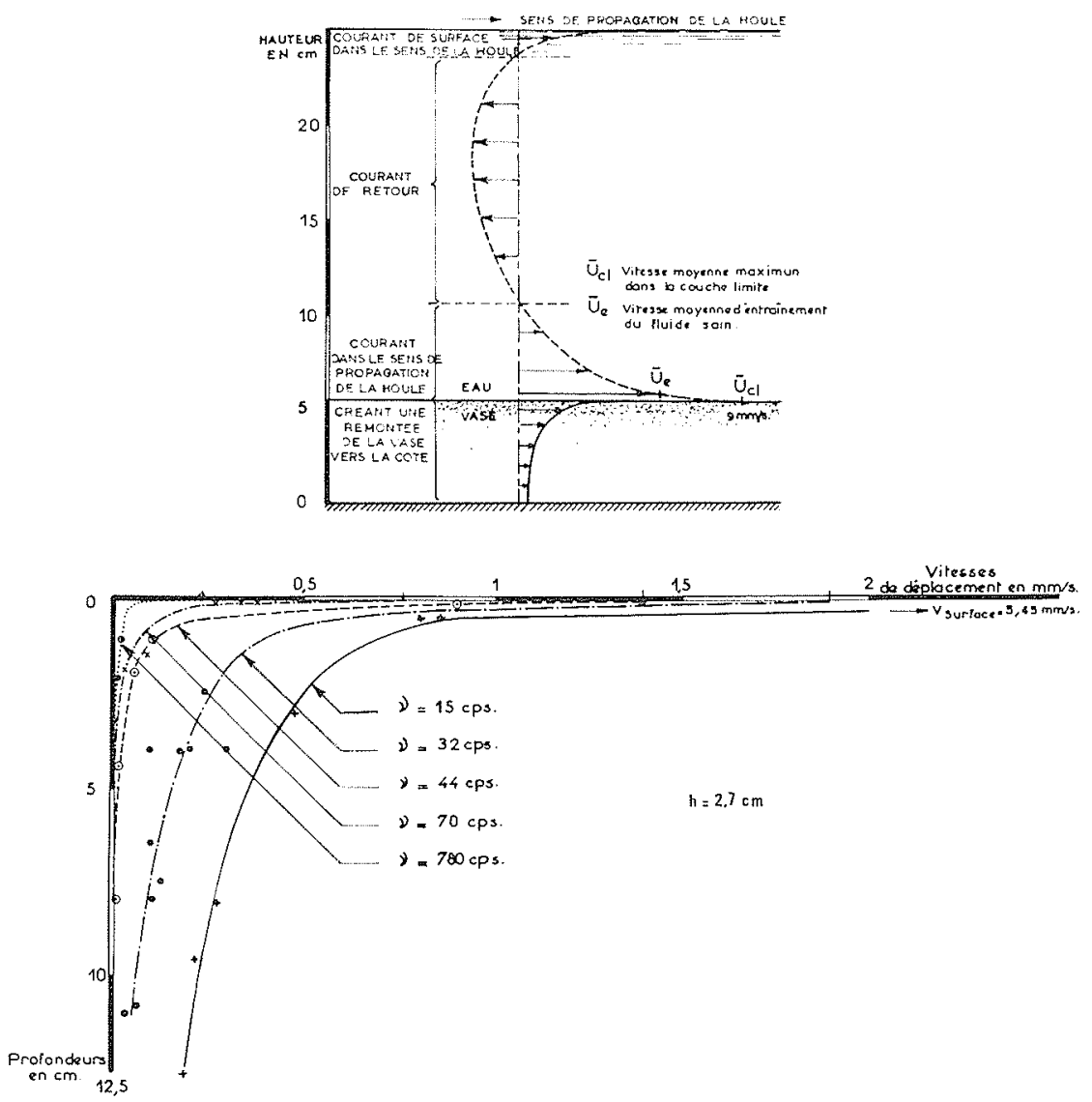

Figure 49 - Déplacement d'une vase fluide ou plastique sous l'action de la houle

$U_{c l}$ étant donné par la formule :

$$
U_{c l}=0,18 U_{0}^{1,6} H^{-0,6} \text { en unités c.g.s.; }
$$

- le débit solide de vase entrainé vers la côte par le mouvement de translation des vases sous l'action de la houle est inversement proportionnel à la viscosité des vases ;

- la vitesse de déplacement augmente avec l'amplitude de la houle.

En pratique, le mouvement de la couche limite dans le sens de propagation de la houle provoque une remontée à la côte des matériaux jusqu'à une zone où la turbulence est suffisamment élevée pour qu'il y ait remise en suspension. Il faut toutefois noter que la propagation du mouvement sur fond déformable augmente la stabilité de l'interface et retarde l'apparition de turbulence de fond ; dans des cas limites la houle ne pourra plus déferler mais viendra mourir au rivage dans un fluide visqueux.

\section{b) Observations en nature}

Les mouvements des fonds vaseux ont été observés en nature par diverses missions du Service Hydrographique du L.C.H.F. et hollandaises le long des côtes guyanaises.

Dès 1875 des missions du Service Hydrographique faisaient mention du déplacement des bancs de vase le long des côtes guyanaises et en 1937 le Comman- dant Yayer indiquait qu'il s'agissait de vases très mobiles glissant sur des vases consolidées et que les houles avaient une action importante sur ces déplacements. Il avait observé également des alternances de vases molles et de vases dures tout le long des côtes Guyanaises sur lesquelles les houles se propageaient différemment.

Les missions du L.C.H.F. entre 1947 et 1957 montraient que ces bancs de vase en se déplaçant entrầnaient des sédimentations importantes des fonds, dépassant $2 \mathrm{~m}$ devant les rivières de Cayenne et de $\mathrm{Ma}$ hury, suivies par des érosions lorsque le banc avait été entraîné en dehors de la zone considérée ; il s'agissait en quelque sorte d'un phénomène cyclique.

Des précisions étaient apportées plus récemment par une mission d'étude hollandaise qui confirmait qu'il existait tout le long des côtes guyanaises des bancs de vase molle de 20 à $30 \mathrm{~km}$ de longueur, dis tants à peu près de la même valeur entre eux. Ces bancs qui se succèdent tout le long des côtes des Guyanes, depuis l'Amazone, progressent de 1 à $1,5 \mathrm{~km}$ par an vers le N.W. Le mouvement s'effectue sous l'action des houles et des courants résiduels le long de la côte avec une direction prédominante vers le N.E.

En 1965 enfin, une mission du L.C.H.F. a pu mesurer avec précision le déplacement d'un front de vase molle sous l'action des houles, apportant des confirmations sur la remontée et la translation des vases par les houles. 


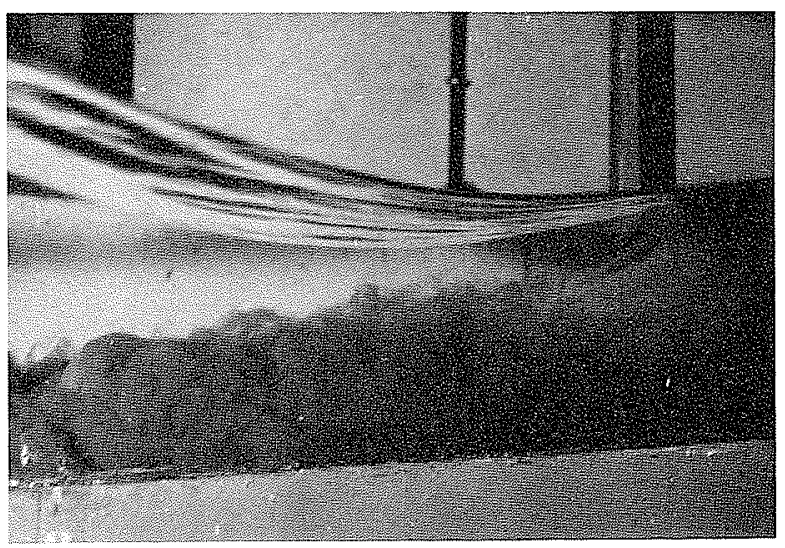

Figure 50 - Remise en suspension des vases au déferlement

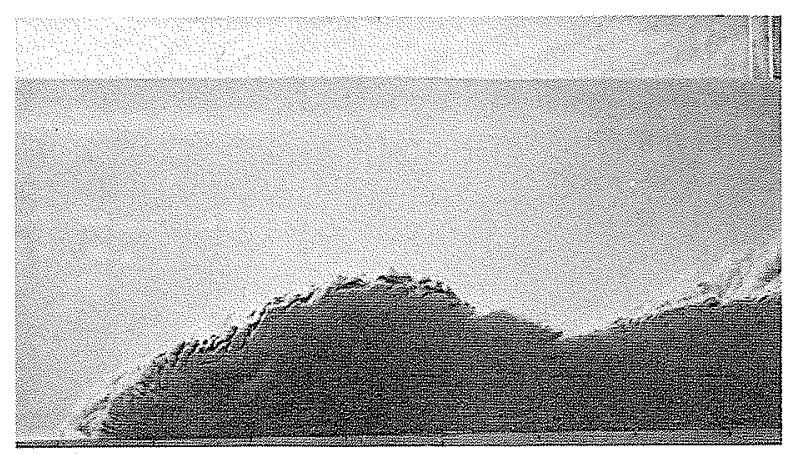

Figure 51 - Courant de turbidité

Une fois dans la zone de déferlement, ou le long des plages, les vases remontées par les houles depuis la zone des petits fonds seront remises en suspension (figure 50) et pourront être réentraînées par les courants littoraux ou s'écouler vers le large sous forme de courants de turbidité (figure 51). On assistera donc le long des plages à un phénomène de lévigation avec ségrégation des sédiments les plus gros, qui pourront à la limite former des plages de sable, et élimination des particules les plus fines avec des concentrations d'autant plus importantes que les houles seront plus fortes.

\section{Mouvements des matériaux parallèlement au rivage}

Les mouvements de matériaux le long du littoral sont des processus fondamentaux à considérer tant dans le diagnostic du régime de la côte que pour l'édification d'un ouvrage maritime susceptible d'interrompre tout ou partie du transit. D'une façon générale une côte sera en érosion, équilibre ou sédimentation selon que les quantités de matériaux traversant la section de la plage sont supérieures, égales ou inférieures à la quantité y pénétrant.

Pour les sédiments non cohésifs du type sables, graviers ou galets il existe un nombre d'études appréciable permettant de fixer les processus de transport et leur ordre de grandeur.

Pour les sédiments très fins du type vases, limons ou argiles les évaluations sont beaucoup plus délicates et si la majeure partie du transport s'effectue en suspension dans le courant littoral on ne peut pas exclure totalement des mouvements plus complexes dans la masse visqueuse que forment les vases fluidifiées par l'action des houles.

\section{Matériaux non cohésifs du type sables et graviers}

\section{a) Description du phénomène}

Pour les sables et notamment pour les sables fins rencontrés sur certaines côtes, il existe deux types de transport littoral différent. L'un sous l'action des houles fortes de tempêtes s'effectue principalement en suspension ( $75 \%$ de transport) l'autre sous l'action des houles faibles de beau temps correspond à un transit mixte en "dents de scie" sur la plage et dans le rouleau de déferlement qui prend naissance en bas de plage.

Le premier type de transit littoral en suspension dans le courant de houle au cours des tempetes peut atteindre des centaines de milliers de $\mathrm{m}^{3}$ sans que le phénomène soit visuellement contrôlable, hormis la coloration des eaux, et sans que le transit ne soit arrêté par des obstacles de petites dimensions (épis, prises d'eau ...) établis entre la côte et les fonds de $-3 \grave{a}-4 \mathrm{~m}$.

Le deuxième au contraire est facilement décelable et contribue aux accumulations sédimentaires le long des ouvrages côtiers de faibles longueurs mais intéresse en général de faibles volumes de sédiments.

D'après les mesures faites par le L.C.H.F. en 1960, le maximum de turbidité, c'est-à-dire le maximum de particules en suspension dans les eaux, se rencontre au point de déferlement de la houle et diminue rapidement de part et d'autre de ce point. Il découle de cette constatation que la plus grande partie du transit littoral en suspension s'effectue légèrement au large de la zone des vitesses maximales du courant de houle. Autrement dit, le transport en suspension s'effectue principalement dans la zone de la barre, par fonds de -6 à $-7 \mathrm{~m}$ par exemple, alors que dans la fosse de lévigation, située entre la barre et la côte, le courant de houle bien qu'encore très violent est relativement peu chargé en particules solides. Pour caractériser ce courant moins turbide du courant général de houle, nous l'avons appelé "courant de lévigation" ; il s'agit d'un courant ayant une puissance érosive d'autant plus élevée qu'il n'a pas une charge sédimentaire très importante.

\section{b) Transport par jet de rive}

Sous l'action de la projection d'eau sur l'estran, ou jet de rive, produite par le déferlement de la houle, les matériaux sont remontés dans la direction de la lame ; lors du reflux par contre, l'eau en redescendant le long de la plage sous l'effet de la pesanteur réentraîne les matériaux suivant la ligne de plus grande pente.

Pour les houles légèrement obliques les particules vont donc être soumises à des mouvements alternatifs de montée et de descente avec une résultante dans le sens préférentiel du transit parallèlement au rivage. Ce transport en "dents de scie" ne représente en géné- 
ral qu'une faible partie des transports longitudinaux dus à l'action de la houle. Pour des sables il ne représenterait que le cinquième ou le dixième du transport total ; par contre pour des galets il peut représenter la quasi totalité du transport par suite de la position des galets sur l'estran.

A titre d'exemple, sur la côte des Bas Champs (Somme) le déplacement moyen des galets a pu être évalué à $1 \mathrm{~km}$ en 6 mois, avec un déplacement maximum de $1,5 \mathrm{~km}$, mais en 15 jours de tempête ( $1^{\mathrm{er}}$ au 15 Décembre 1965) le transport avait atteint $270 \mathrm{~m}$ soit 10 à $15 \mathrm{~m}$ par jour environ.

Le transport s'effectuait principalement dans le tiers supérieur du profil du cordon avec un débit moyen de 80 à $100 \mathrm{~m}^{3} /$ jour. En dehors de la zone d'estran le transport des galets était faible à nul.

D'une façon générale les mesures faites en nature par le L.C.H.F. sur les côtes de la Manche montrent que le débit journalier de galets croît linéairement comme le carré de l'amplitude de la houle et avec son obliquité. Ce même résultat a été obtenu par Zhdanow (Figure 52).

\section{c) Transport au voisinage du déferlement}

La zone de déferlement correspond à un véritable pôle d'attraction sédimentaire vers lequel les sédiments du large peuvent être remontés et ceux du littoral redescendus pour certaines caractéristiques des vagues. Par suite de la concentration de l'énergie des houles et de la forte turbulence les sédiments sont remis en suspension et brassés par les vagues, la plus grande partie des transports s'effectuant dans le premier tiers de la distance comprise entre les brisants et la côte (figure 53).

Si l'obliguité de la houle avec le rivage est appréciable il se crée au voisinage du déferlement un courant de houle capable d'entraîner parallèlement au littoral une masse importante de sédiments en suspension et en charriage créant un débit solide qui constitue, dans le cas des sables, le mode de transport prédominant dû à la houle.

Des mesures systématiques faites par le L.C.H.F. en Méditerranée et en modèle réduit ont permis de quantifier l'importance de ces mouvements sédimentaires aux approches du déferlement.

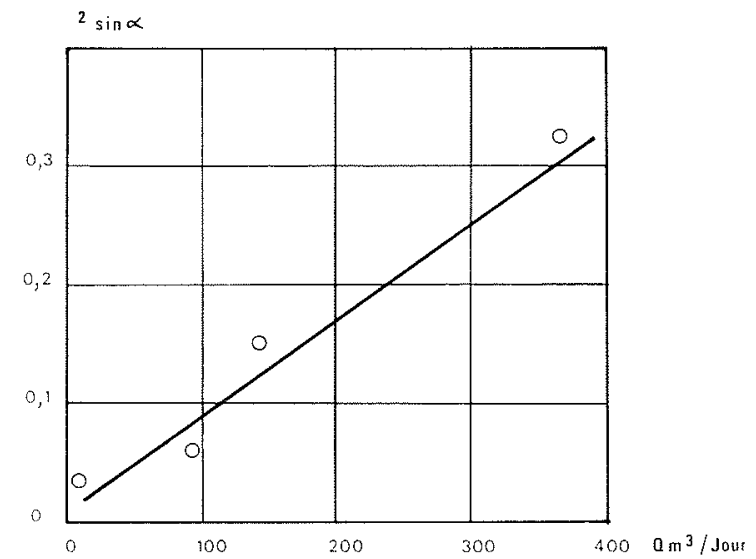

Figure 52 - Transit des galets en fonction de l'amplitude $h$ et de l'angle $\alpha$ d'approche de la houle

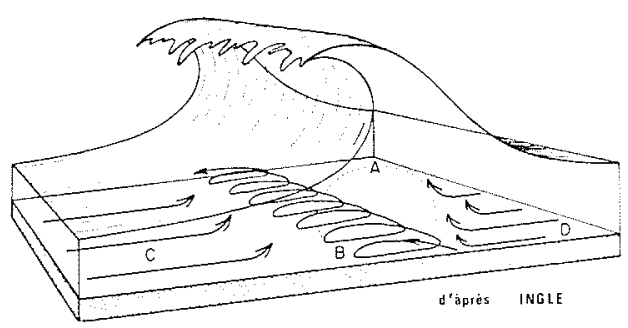

Figure 53 - Schéma du mouvement des grains dans les brisants
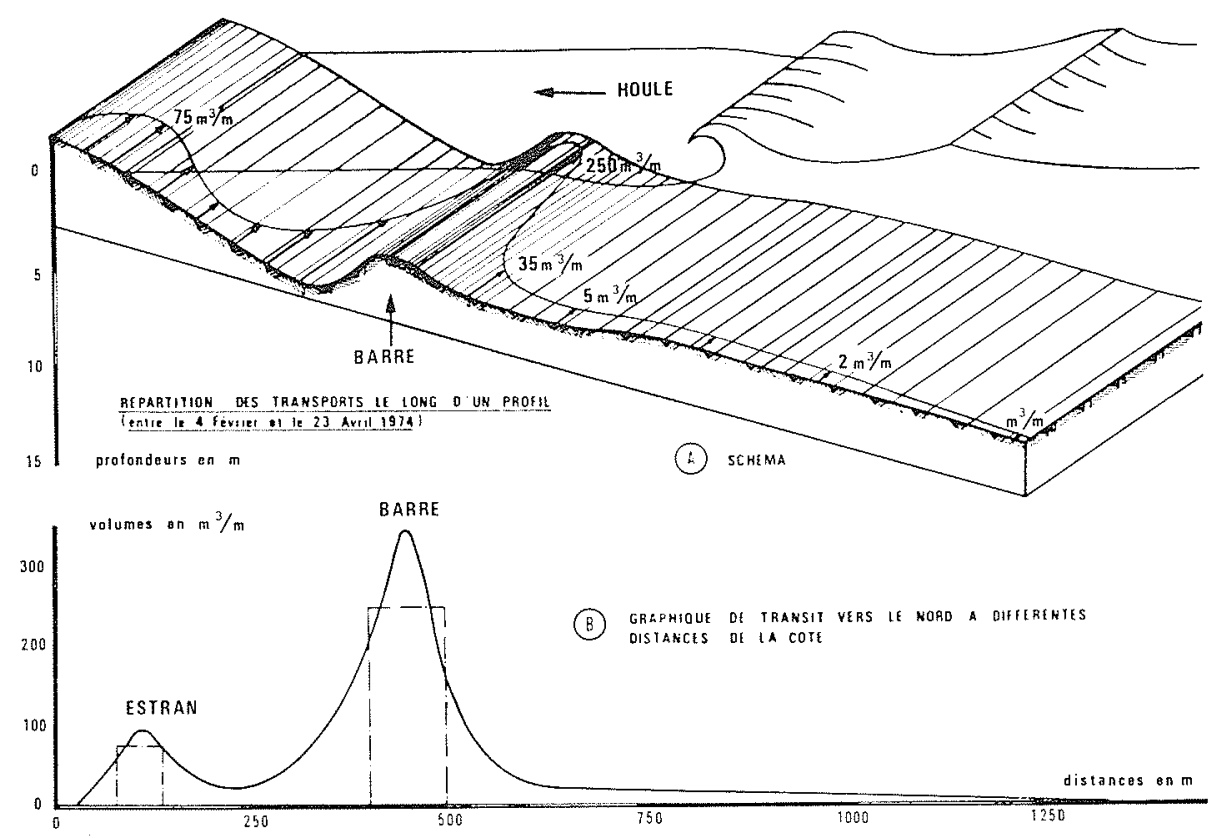

Figure 54 - Transit sédimentaire parallèle au littoral en Méditerranée 
En nature, les expériences faites à l'aide de traceurs radioactifs en association avec la SAPRA et l'A.I.E.A. (Agence Internationale de l'Energie Atomique) entre le 4 Février et le 23 Avril 1974 ont permis d'évaluer le transport résultant parallèle à la côte. La pente moyenne des fonds était de 1/80 et les sédiments constitués de sables fins de 0,25 à $0,10 \mathrm{~mm}$. Les houles comportaient des amplitudes significatives dépassant $3 \mathrm{~m}$, le bilan de transport vers le Nord étant de $12,510^{6} \mathrm{~m}^{2} . \mathrm{S}^{2}$ (Somme de $h^{2}$ Tt $f(\alpha)$ pendant 80 jours de mesures).

La répartition des transports parallèlement au littoral est donnée sur la figure 54 sur laquelle nous avons fait figurer les valeurs du transit mesuré à l'aide des traceurs radioactifs.

On constate que :

- par fonds de - $15 \mathrm{~m}$ il n'y a pas de transport appréciable ( $1 \mathrm{~m}^{3} / \mathrm{m}$ environ en 80 jours);

- par fonds de - $12 \mathrm{~m}$ on a pu observer une dérive oblique se traduisant par une remontée vers la côte et un déplacement parallèle au rivage atteignant $2 \mathrm{~m}^{3} / \mathrm{m}$;

- par fonds de $-8 \mathrm{~m}$ ce phénomène s'accentue et atteint $15 \mathrm{~m}^{3} /$ an parallelement au rivage pour passer à $35 \mathrm{~m}^{3} / \mathrm{m}$ par des fonds de -6 à $-7 \mathrm{~m}$;

- dans la barre et sur sa face interne le débit solide atteint $250 \mathrm{~m}^{3} / \mathrm{m}$ en 80 jours ;

- après être rediminué à $25 \mathrm{~m}^{3} / \mathrm{m}$ dans la fosse de lévigation, située à $250 \mathrm{~m}$ de la barre vers la terre, le débit remonte ensuite à $75 \mathrm{~m}^{3} / \mathrm{m}$ dans la zone d'estran ;

- près de $75 \%$ du transit littoral s'est effectué au voisinage du déferlement et notamment sur sa face interne ;

- le coefficient de transport $K$ de sable (granulométrie comprise entre $0,25 \mathrm{~mm}$ à l'estran et $0,15 \mathrm{~mm}$ au large) atteint des valeurs comprises entre 3,3 et $5.10^{-6}$ alors que les estimations du L.C.H.F. antérieures donnaient une valeur moyenne de $4.10^{-6}$.

Notons que le coefficient $K$ est celui donné par la formule de transport littoral :

$$
Q=\frac{K g}{c} h^{2} T f(\alpha)
$$

Il faut rappeler également que les particules de sable se déplacent parallèlement au littoral à une vitesse plus faible que celle du courant de houle. M. Orlava a trouvé que le rapport entre les vitesses du sable et du courant littoral était de 0,60 pour des sables fins de $0,20 \mathrm{~mm}$ et de 0,06 pour des sables grossiers de $2 \mathrm{~mm}$. Ces valeurs n'ont pu être vérifiées par le L.C.H.F.

En modèle réduit en effectuant des études dans une cuve à houle de $700 \mathrm{~m}^{2}$ de superficie et en reproduisant les amplitudes des houles à l'échelle des hauteurs et les périodes à la racine carrée de cette échelle on a pu schématiser dans de très bonnes conditions les mouvements sédimentaires constatés en nature. Les sables étaient représentés par des particules de bakélite de densité 1,40 et ayant un grossissement de 1,4 par rapport aux diamètres des sables naturels.

En effectuant des mesures systématiques des transports en suspension et en charriage sur les fonds on a pu constater que :

- la plus grande quantité en transit s'effectue, pour des sables fins, au voisinage du déferlement;

- en tempête près de $75 \%$ des sables fins transportés parallèlement au littoral sont déplacés en suspension dans le courant littoral non pas au point où sa vitesse est maximum, mais sur la face interne de la barre (figure 55);

- le coefficient de transport de la bakélite est 25 à 30 fois supérieur à celui du sable naturel, cette évaluation permettant de déterminer théoriquement l'échelle des temps sédimentologique d'un modèle physique.

Ces deux résultats en nature et en modèle réduit sont à rapprocher de ceux donnés par Zenkovitch en 1958 qui a étudié la répartition des débits solides le long d'une côte comportant une double barre pour des houles ayant respectivement 0,5 à $1 \mathrm{~m}$ et 2 à $3 \mathrm{~m}$ d'amplitude et dont l'obliquité induit des courants de 0,20 à $0,60 \mathrm{~m} / \mathrm{s}$. Zenkovitch montrait que $60 \%$ du transport en suspension s'effectuait au voisinage du premier rouleau de déferlement avec des turbidités moyennes de 3 à $4 \mathrm{~g} / 1,20 \%$ de transport ayant lieu au voisinage de la deuxième barre et $6 \%$ seulement sur l'estran bien que l'on puisse y mesurer des concentrations de 15 à $30 \mathrm{~g}$ de sables par litre d'eau prélevée (figure 56).

\section{d) Estimation du transit littoral}

Le transit littoral en un site donné peut être évalué suivant plusieurs méthodes:

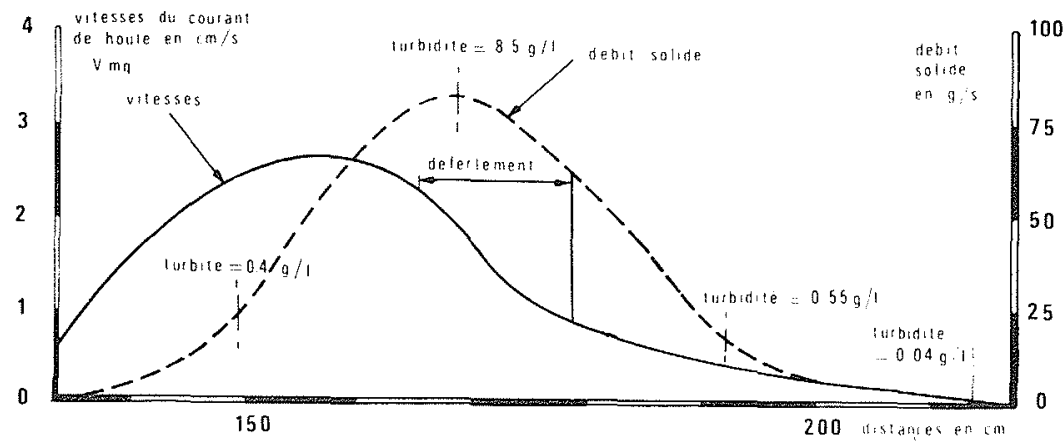

Figure 55 - Répartition du transit littoral le long d'un profil sur modèle réduit 


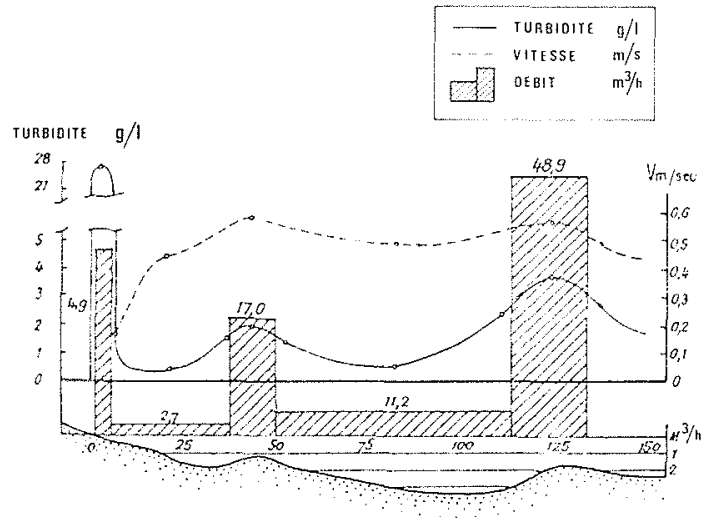

Figure 56 - Vitesse du courant en surface et débit solide par houle de 2 à $3 \mathrm{~m}$ d'après Zenkovitch

- la première consiste à évaluer le transit littoral de l'évolution des fonds au cours du temps, comme par exemple en suivant les volumes de matériau arrêtés par un ouvrage. Il faut s'assurer dans cette méthode que les ouvrages arrêtent effectivement la totalité du transit et non une partie seulement comme le ferait un épi. Cette méthode n'est pas applicable en site vierge ;

- la deuxième méthode consiste à utiliser des valeurs bien connues en un site voisin, en introduisant éventuellement des corrections basées sur les conditions locales; On peut également, par analogie avec des littoraux ayant des caractéristiques océanographiques, topographiques et sédimentologiques voisines, essayer d'avoir des ordres de grandeur du transit littoral; malheureusement une situation aussi favorable ne se présente que rarement ;

- les traceurs radioactifs et les mesures de turbidité des eaux - vitesses des courants, seraient une troisième méthode. Pour l'appliquer sur une année entière cette méthode est assez longue et demande des investissements appréciables ;

- on peut enfin utiliser des formules ou des abaques permettant d'évaluer le transit littoral pour différentes actions de houles et en faire le bilan annuel.

Ces formules font intervenir une approche empirique très importante, la complexité des phénomènes ne permettant pas de les traiter de façon purement théorique. Dans la formule utilisée au L.C.H.F. on a essayé, après avoir contrôlé en modèle réduit l'importance à donner aux différents paramètres, de définir des coefficients de transport des sédiments à partir des mesures faites directement en nature.

Différentes études ont montré que le transit littoral de sables fins dépendait du bilan de transport de la houle et pouvait être rattaché à une expression de la forme :

$$
V=\Sigma h^{2} T f(\alpha) t\left(\frac{K g}{c}\right)
$$

avec :

$V=$ volume en $\mathrm{m}^{3}$ de sédiments transportés

$h=$ amplitude maximale en $\mathrm{m}\left(h_{1 / 10}\right)$ de la houle

$T=$ période moyenne de la houle en seconde $\alpha=$ obliquité des vagues avec le littoral par fonds $\mathrm{de}-15 \mathrm{~m}$

$t=$ temps d'action d'une houle de caractéristique donnée.

Le terme $\mathrm{Kg} / \mathrm{c}$ caractérise le coefficient de transport du sable. L'influence de la cambrure $C$ mise en dénominateur n'a pas fait l'objet d'étude systématique en nature, les résultats n'étant valables que pour les cambrures supérieures à $1 \%$.

Sous cette réserve on a pu estimer, à partir de mesures en nature et pour des sables fins siliceux de 0,20 à $0,30 \mathrm{~mm}$, que les valeurs du coefficient $K$ étaient comprises entre 3 et $4,510^{-6}$ (valeurs que des mesures à l'aide de traceurs radioactifs en Méditerranée ont confirmées, comme il a été dit précédemment).

Pour des sables coquilliers plats de $0,5 \mathrm{~mm}$ la valeur de ce coefficient $K$ atteindrait $6,10^{-6}$. Par contre pour les diamètres de sables siliceux supérieurs à $0,3 \mathrm{~mm}$ le coefficient $K$ diminuerait suivant l'expression :

$$
K=1,810^{-6} d^{-1 / 2}(d \text { en } \mathrm{mm})
$$

De même il est vraisemblable que pour les particules fines le coefficient $K$ serait inférieur à $3 \cdot 10^{-6}$, par suite de l'apparition de phénomènes de cohésion.

En Méditerranée où l'on rencontre des houles ayant fréquemment des cambrures de $4 \%$ et des sables fins de 0,20 à $0,30 \mathrm{~mm}$ on peut avoir une idée du transit littoral en adoptant la formule simplifiée :

$$
V\left(\mathrm{en} \mathrm{m}^{3}\right)=10^{-3} \cdot h^{2} \cdot T \cdot \sin \frac{7 \alpha}{4} \cdot t
$$

Par exemple si $h=5 \mathrm{~m}, T=12 \mathrm{~s}, \alpha=15^{\circ}$ et $t=24$ heures le transit littoral serait

$$
\begin{aligned}
& V\left(\text { en } \mathrm{m}^{3} \text { pour } 24 \mathrm{~h}\right)= \\
& \quad=10^{-3} \cdot 25 \cdot 12 \cdot 0,45(24 \times 3.600)=11.600 \mathrm{~m}^{3}
\end{aligned}
$$

Pour simplifier le calcul on trouvera sur la figure 57 un abaque, établi par le L.C.H.F., permettant de déterminer les ordres de grandeur du transport littoral connaissant les caractéristiques de la houle et du matériau.

Il faut également citer les études faites par le CERC qui donnent :

$$
Q 1\left(\text { en m } \mathrm{m}^{3} / \text { jour }\right)=10^{4} h_{d}^{5 / 2} \sin 2 \alpha_{d}
$$

et qui conduit en général à des valeurs du transit littoral supérieures à celles données par le L.C.H.F., ainsi que la formule Larras-Bonnefille qui montre que le maximum de transport est obtenu pour des sables de $0,8 \mathrm{~mm}$ et que pour les cambrures supérieures à $1 \%$ le transport décroit lorsque la cambrure augmente.

A titre d'information, nous indiquons ci-après quelques valeurs du transit littoral annuel mesuré en nature dans différents sites et des indications sur les quantités transportées au cours d'une tempête ou d'une courte période :

- Safi (Maroc) : transit annuel $250.000 \mathrm{~m}^{3}$, mais une tempête de 3 jours peut, à elle seule, transporter $80.000 \mathrm{~m}^{3}$ de sable (2 au 4 Janvier 1949); 


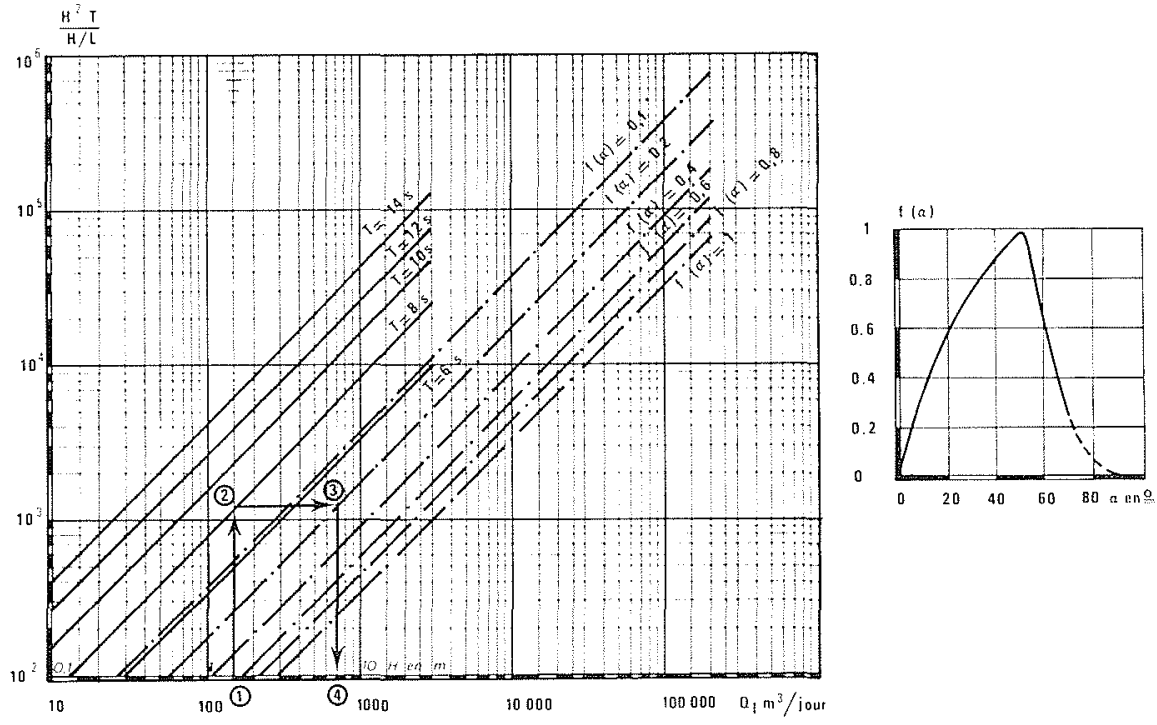

Figure 57 - Transit littoral en fonction des caractéristiques de la houle (d'après la formule en usage au L.C.H.F.)

- Agadir (Maroc) : transit annuel 450.000 à $500.000 \mathrm{~m}^{3}$, mais une houle forte à moyenne $(h=1,5$ à $2,5 \mathrm{~m})$ peut transporter $150.000 \mathrm{~m}^{3}$ en 15 jours ; en 7 heures une houle de $2,5 \mathrm{~m}$ d'amplitude et $12 \mathrm{~s}$ de période a transporté $35.000 \mathrm{~m}^{3}$ les 29 et 30 Novembre 1947 ;

- Pointe Noire (Congo) : transit annuel $400000 \mathrm{~m}^{3}$ dont $300000 \mathrm{~m}^{3}$ apportés au cours des fortes tempêtes ;

- Côte du Benin : transit annuel $1500000 \mathrm{~m}^{3}$;

- Tanger : transit annuel de $60000 \mathrm{~m}^{3}$;

- Méditerranée Orientale : transit annuel variant de $800000 \mathrm{~m}^{3}$ par an à $50000 \mathrm{~m}^{3}$ lorsque l'on se déplace du Sud vers le Nord (obliquité variable de la côte). Près de $80 \%$ du transit s'effectue au cours des tempêtes supérieures à $4 \mathrm{~m}$, c'est-à-dire en moins de un mois ;

- Côte de Manche (Lion S/Mer) ; transit annuel $50000 \mathrm{~m}^{3}$ (évaluation);

- Nord de l'Adour : transit annuel $300000 \mathrm{~m}^{3}$ (évaluation) ;

- Côte des Bas Champs (galets) $20000 \mathrm{~m}^{3} /$ an avec déplacement des galets de $1 \mathrm{~km}$ par an environ.

Ces valeurs montrent la diversité du transit littoral. Dans tous les cas on doit se rappeler que ce transit s'effectue au voisinage du déferlement c'est-à-dire à des profondeurs variables, pouvant atteindre plusieurs mètres au cours des tempêtes, et qu'il peut passer devant un rivage sans être arrêté par des ouvrages courts tels que les épis.

Mouvements des matériaux cohésifs, du type vase, parallèlement au rivage

Il est très difficile d'évaluer l'importance du transit littoral d'éléments pélitiques du type vases ou limons, sous la seule action des houles obliques. Dans de nombreux cas en effet le déplacement des particules fines le long de la côte s'effectue sous la double action des courants de marée et des courants de houle sans que l'on puisse en séparer leur influence propre.

On a vu dans le paragraphe relatif au mouvement des particules perpendiculairement au littoral que les houles pouvaient remanier les fonds de vase et remonter une partie des matériaux à la côte. Si il y a déferlement de la houle les vases seront remises en suspension et entraînées dans le sens du courant, qu'il s'agisse d'un courant issu de l'obliquité des houles ou d'un courant de marée. Les mesures faites en nature montrent que la turbidité des eaux va croître très rapidement avec la force des houles et passer de quelques dixièmes de grammes à plusieurs grammes de sédiments secs par litre comme Bouquet de la Grye l'avait déjà montré au siècle dernier au cours des mesures hydrographiques à La Rochelle (la turbidité aurait augmenté comme le carré des amplitudes).

$\mathrm{Si}$ les vases sont très fluides la houle pourra très bien venir mourir à la côte sans déferler par suite de la destruction de son énergie dans un fluide à forte viscosité. Les déplacements parallèles au rivage s'effectueront alors en masse. Le L.C.H.F. a pu, sur les côtes de Guyane, mesurer en 1954 une dérive de vase molle sous l'action de la houle atteignant $400 \mathrm{~m}$ en 12 jours.

Dans certaines zones du globe les mouvements de vase parallèlement au littoral sont très importants, il suffit de rappeler les dépôts de 40 millions de $\mathrm{m}^{3}$ de vase en 6 ans devant l'embouchure de la rivière à Cayenne (vase d'origine marine) pour en avoir une idée.

Même le long des côtes du littoral français où les problèmes d'envasement peuvent apparaître comme plus faibles et où la turbidité des eaux $d u$ large semble négligeable on constate que sous les actions conjuguées de la houle et des courants on a dans les ports et les chenaux des valeurs d'envasement très appréciables dont il faut tenir compte.

En nous basant sur des apports uniquement marins on trouve à titre d'exemple les valeurs suivantes de l'envasement : 
- fond du bassin de La Rochelle (soumis à des chasses) : $0,30 \mathrm{~m}$ par an ;

- port de Pornic : 0,50 m par an en moyenne ;

- avant port de Ouistreham : $2 \mathrm{~m}$ par an ;

- port du Havre : $1 \mathrm{~m}$ par an dans certaines zones;

- port de Dieppe : 1,25 à 1,50 m par an dans l'avant port ;

- port de Granville : $0,50 \mathrm{~m}$ par an ;

- ancien port de Dunkerque : 2,15 m par an ;

- nouvel avant port de Dunkerque : $0,40 \mathrm{~m}$ par mois pendant les travaux ;

- port de Zeebruge : $3 \mathrm{~m}$ par an ;

- port de St Nazaire : 0,25 à $0,50 \mathrm{~m}$ par mois.

11 est bien évident que la houle seule n'est pas à l'origine de l'envasement de ces ports maritimes de la côte atlantique et que les actions des courants de marée sont pour beaucoup dans de tels phénomènes. Il n'en reste pas moins vrai que, très souvent, les taux de sédimentation mesurés en nature sont supérieurs, et très nettement supérieurs, à ceux que l'on aurait pu évaluer par des mesures de la turbidité des eaux par houle moyenne et faible, même en admettant que la totalité des eaux qui pénétrent dans un bassin se décantent au cours d'une étale de courant.

Notons que ces valeurs du taux de sédimentation en zone purement maritime sont inférieures aux taux d'envasement que l'on pourrait mesurer en estuaire et qui peuvent dépasser dans certains cas $1 \mathrm{~m}$ par mois (La Houille Blanche $n^{\circ} 1 / 2-1974$ ).

11 n'en reste pas moins que les problèmes des apports en éléments très fins du type vase dans les zones portuaires ne doivent pas être négligés même si par quelques mesures rapides en période de houle faible on constate que la turbidité des eaux est peu élevée.

\section{Comportement des sédiments sous l'action du vent}

\section{Généralités}

Le transport des sédiments sableux par le vent peut avoir une influence non négligeable aux abords d'un port et certains franchissements d'ouvrages maritimes par les mouvements éoliens entraînent des sédimentations qu'il est nécessaire de pouvoir évaluer. De même le long d'une plage le transport des sables vers l'intérieur des terres est une source d'appauvrissement sédimentaire du littoral qui peut atteindre des centaines de milliers de $\mathrm{m}^{3}$ provoquant la formation de dunes comme le long de la côte des Landes en France ou du littoral marocain au Sud d'Agadir ; en contre partie le transit littoral se trouvant sous alimenté par la perte en matériau des plages, une érosion des plages sous-jacentes peut en résulter.

\section{Mesures effectuées en nature}

$\mathrm{Au}$ cours de différentes missions le L.C.H.F a eu l'occasion de mesurer l'importance du transport des sables fins par les vents. Ces mesures étaient réalisées en disposant le long de mâts fichés en terre de petits pièges à matériaux et en mesurant aux mêmes points la vitesse du vent.

Quelques valeurs relevées en nature par le L.C.H.F. à Tanger en 1950 (figures 58 et 59) illustrent l'importance des transports éoliens le long des plages et la nécessité d'en tenir compte dans l'évaluation des mouvements sédimentaires. Il faut évidemment dans une telle étude tenir compte des statistiques de pluie et d'ensoleillement qui modifient dans de larges mesures les possibilités de mise en mouvement des particules solides.

En intégrant les mesures sur toute la hauteur audessus du sol on arrivait dans le cas de Tanger aux valeurs suivantes :

\begin{tabular}{|c|c|c|c|c|c|c|}
\hline $\begin{array}{c}\text { Emplacement des } \\
\text { mesures }\end{array}$ & \multicolumn{5}{|c|}{$\begin{array}{c}\text { TANGER - Vitesses à } 2 \mathrm{~m} \\
\text { au-dessus du sol }\end{array}$} \\
\hline Vitesses du vent (en m/s) & 11,1 & 10,2 & 8,3 & 7,8 & 7,1 & 6,5 \\
\hline $\begin{array}{c}\text { Quantités transportées } \\
\text { en } \mathrm{kg} / \mathrm{m} / \mathrm{h}\end{array}$ & 145 & 57 & 16,8 & 14 & 4 & 2,8 \\
\hline
\end{tabular}

\section{Formules du transport éolien}

La formule dite de Bagnold fournit un ordre de grandeur du débit solide traversant un plan vertical de largeur unité, perpendiculaire à la direction du vent.

\section{Début de mouvement}

Partant de la vitesse de frottement sur les fonds $\left(u_{*}=\sqrt{\tau / \rho}\right)$ Bagnold démontre que la vitesse minimale pour qu'il puisse y avoir transport s'écrit :

$$
u_{*_{c}}=A \sqrt{\frac{\rho_{s}-\rho}{\rho} g d}
$$

$u_{*_{c}}=$ vitesse de frottement critique (en $\mathrm{m} / \mathrm{s}$ )

$A=0,1$

$\rho_{s}=$ masse volumique des grains $2600 \mathrm{~kg} / \mathrm{m}^{3}$

$\rho=$ masse volumique de l'air $1,292 \mathrm{~kg} / \mathrm{m}^{3}$

$g \quad=9,81 \mathrm{~m} / \mathrm{s}^{2}$

$d=$ diamètre des grains en $\mathrm{m}$

Pratiquement on peut prendre pour du sable :

$\begin{array}{lll} & u_{c^{*}}=0,45 \sqrt{d} & (d \text { en mm }) \\ \text { ou } & \\ & u_{c^{*}}=14,3 \sqrt{d} & (d \text { en } \mathrm{m})\end{array}$

pour obtenir $u_{*_{c}}$ en $\mathrm{m} / \mathrm{s}$ 


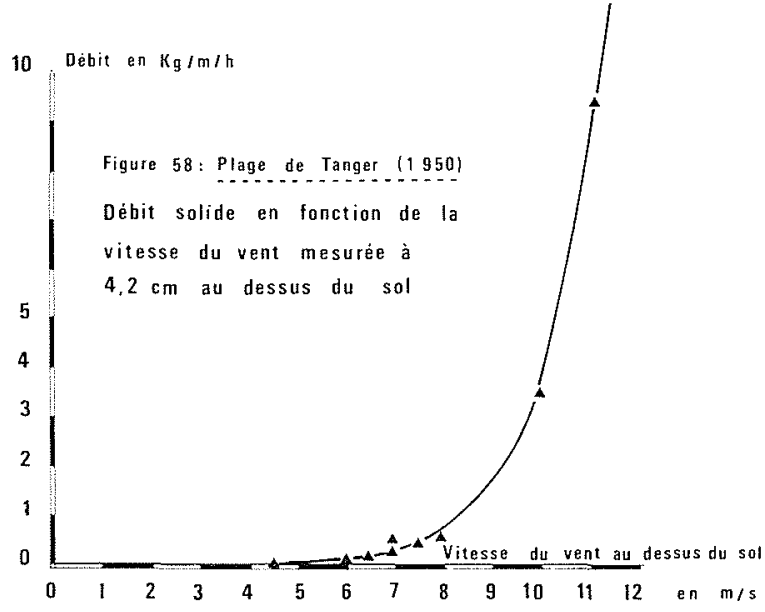

Figure 58 - Délit solide en fonction de la vitesse du vent mesuré à $4,2 \mathrm{~cm}$ du sol

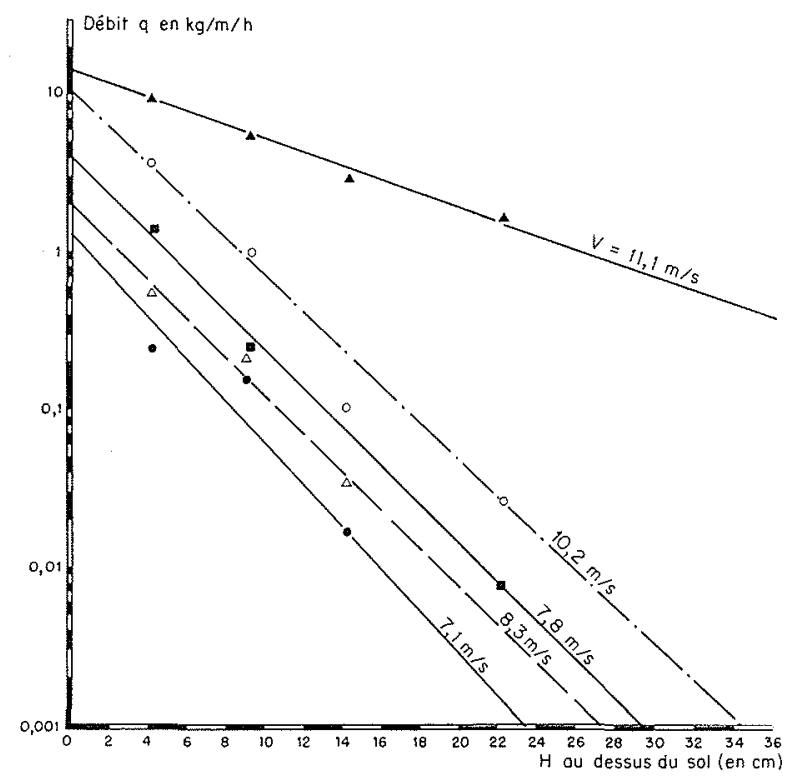

Figure 59 - Délit solide en fonction de la hauteur en dessus du sol et de la vitesse du vent

\section{Détermination de la vitesse de frottement sur le sol}

La détermination de $u_{*}$ dans le cas où il y a transport éolien pourra se faire à partir de mesures des vitesses sur la verticale à différentes distances $Z$ du sol :

$$
u_{*}=\frac{V(Z)-V(Z f)}{K \log _{10} \frac{Z}{Z f}}
$$

avec $Z f=12 d$ (sable très uniforme) et $Z f=40 d$ (sable très dispersé)

La donnée de $u_{*}$ est intéressante car elle permet de déduire la vitesse minimale du vent, mesurée à une cote $\mathrm{Z}$ donnée, telle qu'il puisse $\mathrm{y}$ avoir transport établi $\left(U_{\mathrm{c}}(Z)\right)$ :

$$
V_{c}(Z)=2,59 \sqrt{d} \log _{10} \frac{30 Z}{d}
$$

si $V_{c}(Z)$ en $\mathrm{m} / \mathrm{s}, Z$ et $d$ en $\mathrm{mm}$

ou :

$$
V_{c}(Z)=82,2 \sqrt{d} \log _{10} \frac{30 Z}{d}
$$

si $V_{c}(Z)$ en $\mathrm{m} / \mathrm{s}, Z$ et $d$ en $\mathrm{m}$
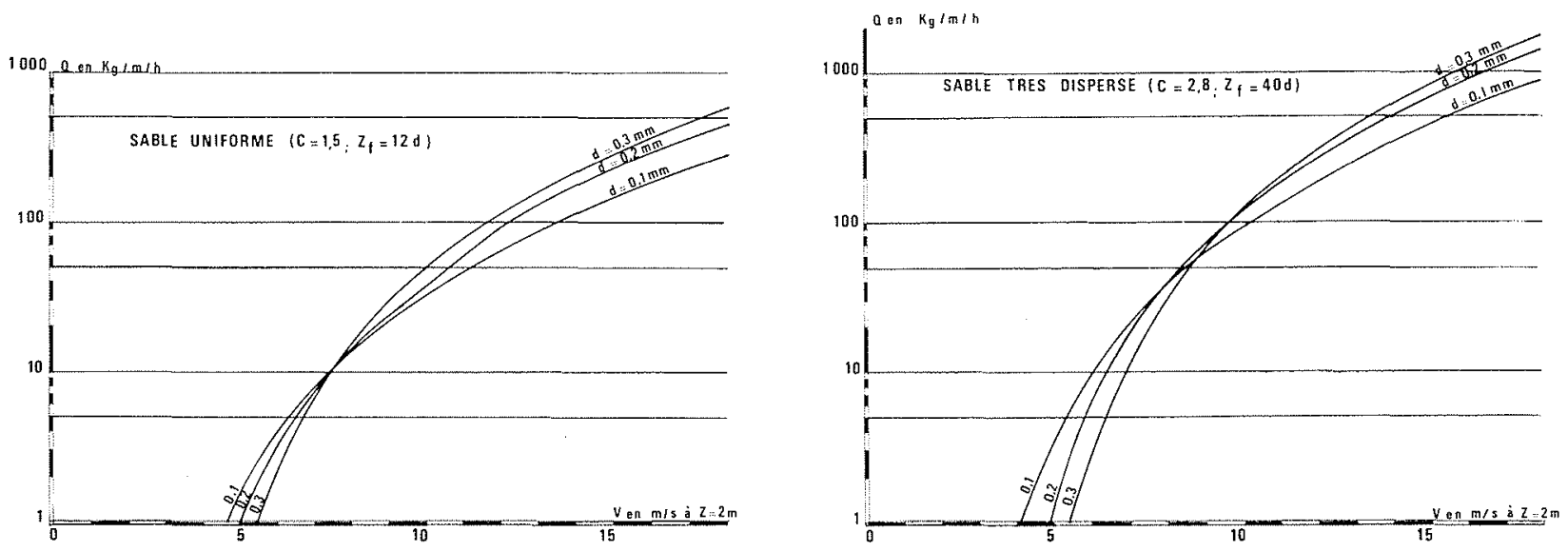

Figure 60 - Délit solide éolien pour différentes vitesses du vent 


\section{Débit solide transporté par le vent}

Le débit solide par unité de largeur s'exprime par la formule :

$$
q=\frac{C \rho}{g} \sqrt{\frac{d}{D}} u_{*}^{3}
$$

$C=$ Constante de Bagnold $=1,5$ pour sable uniforme à 2,8 sable très dispersé,

$D=$ diamètre de référence $0,25 \mathrm{~mm}$,

$u_{*} \approx$ définie précédemment (vitesse de frottement correspondant au régime de transport).

D'une façon pratique, en passant aux valeurs numériques, on prendra pour évaluer le débit éolien en $\mathrm{kg} / \mathrm{m}$ heure la formule :

$$
q=854 \sqrt{\frac{d}{0,25} u_{*}^{3}}
$$

avec $d$ en mm et $u_{*}$ en $\mathrm{m} / \mathrm{s}$.

Les abaques de la figure 60 permettent, connaissant la vitesse à $2 \mathrm{~m}$ du sol de définir le débit solide de différents sables fins $(d=0,1$ à $0,3 \mathrm{~mm})$ pour différentes dispersions du matériau.

\section{Conclusions générales}

Le recueil des formules et abaques donnés dans cet exposé sur l'action des courants, de la houle et du vent sur les sédiments n'a pour but que d'essayer de mettre à la disposition des ingénieurs et techniciens, qui auront à se pencher sur le problème des mouvements sédimen- taires, un certain nombre d'éléments pratiques susceptibles de donner des ordres de grandeur des phénomènes dans des cas relativement simples.

Abandonnant volontairement des considérations purement théoriques sur les mouvements sédimentaires, la priorité a été donnée à la description des phénomènes physiques qui interviennent dans les transports de sédiments. De même, plutôt que de développer des formules très élaborées, on a préféré donner des formules simplifiées facilement exploitables, illustrées par des abaques et des exemples pratiques en nature que le Laboratoire Central d'Hydraulique de France a eu l'occasion d'accumuler depuis plusieurs décennies.

Dire que la vitesse de chute d'un sable dans de l'eau calme est égale à 125 fois son diamètre, ou que celle d'un flocon de vase est voisine de $0,5 \mathrm{~mm} / \mathrm{s}$ peut paraître très simpliste. De même que dire que la pente d'un talus de vase immergée (exprimé par sa tangente) est égale à $1 / 40$ de la rigidité du dépôt (en $N / \mathrm{m}^{2}$ ) ou que la force tractrice critique d'entraînement d'un sable par un courant (en $N / \mathrm{m}^{2}$ ) est égale au diamètre du grain en millimètres et que la vitesse critique de frottement nécessaire pour éroder une vase est égale à 0,03 fois la racine carrée de la rigidité du dépôt. . . peut sembler trop simplifié. Il n'en est pas moins vrai que c'est en utilisant de telles formules que l'on peut rapidement, et pratiquement, aborder les problèmes complexes des mouvements sédimentaires sous les différentes actions hydrodynamiques et éviter ainsi des erreurs importantes.

Ce n'est que dans un deuxième stade de perfectionnement que l'on aura intérêt à aborder les problèmes par des formules beaucoup plus complètes et complexes sans oublier pour autant que pour les recherches sédimentologiques, la véritable méthode d'investigation reste dans les mesures en nature et dans leur extrapolation grâce à des études sur des modèles réduits physiques. 